\title{
A SEARCH FOR NARROW STATES IN RADIATIVE UPSILON DECAYS *
}

\author{
Stephen T. Lowe ${ }^{\dagger}$ \\ Stanford Linear Accelerator Center \\ Stanford University \\ Stanford, California 94305
}

December 1986

\section{Prepared for the Department of Energy}

under contract number DE-AC03-76SF00515

Printed in the United States of America. Available from the National Technical Information Service, U.S. Department of Commerce, 5285 Port Royal Road, Springfield, Virginia 22161. Price: Printed Copy A07, Microfiche A01.

* Ph.D. dissertation.

$\dagger$ Work supported in part by the Department of Energy, contract DE-AC03-76SF00515 and DE-AC03-76SF00098. 


\section{ABSTRACT}

A search for new states produced in radiative $\Upsilon(1 \mathrm{~S})$ decays is accomplished by observing the inclusive photon energy spectrum. A narrow resonance in the energy spectrum indicates the existence of a new state $X$ produced by the process $\Upsilon \rightarrow \gamma X$. The analysis is based on approximately $0.44 \times 10^{6} \Upsilon(1 \mathrm{~S})$ events produced at the DORIS II $e^{+} e^{-}$storage ring. These data were collected with the Crystal Ball detector between April 1983 and May 1986.

This analysis finds no evidence for a new state, so upper limits on the branching ratio $B R(\Upsilon \rightarrow \gamma X)$ are derived, assuming the state $X$ decays primarily to high-multiplicity hadronic final states. In particular, if the state $X$ were a minimal Higgs particle, its primary decay mode would be to the heaviest fermionantifermion pair energetically available. For the radiative $\Upsilon(1 S)$ decays studied here, the heavy fermions would be $c \bar{c}$ or $s \bar{s}$ quark states, over most of the relevant Higgs' mass range.

The resulting upper limit for $B R(\Upsilon(1 \mathrm{~S}) \rightarrow \gamma X)$ is highly energy dependent but for $X$ mass between $1.5 \mathrm{GeV}$ and $8.0 \mathrm{GeV}$, the $90 \%$ confidence level upper limit is better than $8.0 \times 10^{-4}$. For a Higgs' mass near $5.0 \mathrm{GeV}$, the upper limit is about $2.0 \times 10^{-4}$ which is approximately equal to the lowest order calculation for the Wilczek mechanism. The Wilczek calculation with $\mathrm{QCD}$ radiative corrections predict branching ratios below the limits set here for all Higgs' masses. 


\section{ACKNOWLEDGEMENTS}

Experiments in High Energy Physics (HEP) are a collaborative effort and as such require the efforts of many. For their contribution to the experiment and to my education, I sincerely thank all those involved in the Crystal Ball experiment. Special thanks go to my advisor, Elliott Bloom, for his great enthusiasm for physics, his encouragement and guidance over the years, and his constant source of ideas (his editorial talents are also greatly appreciated).

Mastering the broad range of techniques needed in experimental physics requires many instructors. For teaching me the hands-on nitty-gritties of experimental HEP, I thank Gary Godfrey, Bill Lockman, Frank Porter, Bob Clare, John Tompkins, Andreas Schwarz and Susan Cooper. I also want to thank those who shared this learning experience with me, in particular, John Butler, Ray Cowan, Chad Edwards, Gunter Folger, John Gaiser, David Gelphman, Gabi Glaser, Stefan Keh, Roger Lee, Steve Leffler, Harry Nelson, Duncan Prindle, Les Rosenberg, Peter Schmitt, Wim Walk and David Williams.

The work needed to typeset this thesis was considerably reduced and the appearance greatly enhanced by the $\mathrm{T}_{\mathrm{E}} \mathrm{X}$ wizard, Ray Cowan. His open willingness to help out anytime is very much appreciated. I also thank Ingrid Clare for help handling tapes and the data on them, and June Belew for her secretarial assistance.

For his help throughout graduate school, his discussions on Life, God and the world, and his active sense of humor, I want to thank the other member of the Steve doublet, Steve Leffler. Our sharing of survival tactics and strategies helped considerably. 
For her friendship, honesty and teaching me a little about rationality (and putting up with me during shifts), I thank Patty McBride. Exploring the restaurants and swimming pools of Hamburg together made life there enjoyable.

I want to thank the "Friendly as Always" Achim Irion for improving the quality of life both in Hamburg and Palo Alto. His flair for hosting great parties, his skill as a pilot, and his tough determination to spend lunch tanning at DeGuerre pool, even in the face of a slight breeze, will be remembered.

For being good friends and discussion partners, I want to thank the very charming sisters, Alyssa Higashi and Andrea Higashi.

Away from work, I want to thank Janet Atwood and Miguel Vargas for being friends of the highest quality. I also thank Chris Peck for sharing life for the first several years of graduate school and a lot of unforgettable experiences.

For starting me off in the scientific direction I thank Mr. Ray Williams and Dr. Evelyn Banks.

Of course my greatest appreciation is for the love and support I've received from my parents and family. My mother and father, Roberta and Carol Lowe, and my brother, Michael Lowe, deserve my deepest thanks. I also want to recognize the love and encouragement I've received from my grandparents, Winnie and Don Lowary, and Pauline and Cecil Lowe.

Finally, I wish to dedicate this thesis to the memory of Don Lowary. 
Table of Contents

1. INTRODUCTION AND SUMMARY $\ldots \ldots \ldots \ldots \ldots \ldots$

1.1 The Standard Model . . . . . . . . . . . . . . . 1

1.2 The Higgs Sector $\ldots \ldots \ldots \ldots \ldots \ldots$

1.3 Searching for the Higgs Boson in Radiative Upsilon Decays $\ldots 19$

1.4 Experimental Results $\ldots \ldots \ldots \ldots \ldots \ldots \ldots$

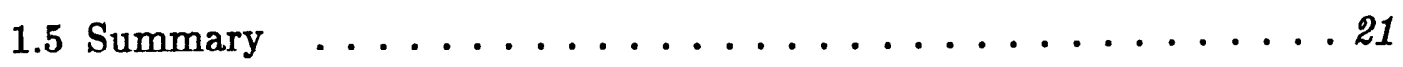

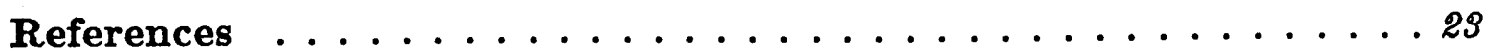

2. THE CRYSTAL BALL EXPERIMENT $\ldots \ldots \ldots \ldots \ldots$

2.1 Introduction . . . . . . . . . . . . 25

2.2 The DORIS II Storage Ring . . . . . . . . . . . 25

2.3 Experimental Overview $\ldots \ldots \ldots \ldots \ldots \ldots \ldots \ldots \ldots$

2.4 The Main Ball $\ldots \ldots \ldots \ldots \ldots \ldots \ldots \ldots$

2.5 Endcaps . . . . . . . . . . . . . . . . 91

2.6 Tracking Chambers $\ldots \ldots \ldots \ldots \ldots \ldots \ldots$. . . . . . . . .

2.7 Luminosity Counters $\ldots \ldots \ldots \ldots \ldots \ldots$. . . . . . . . . . .

2.8 Time of Flight System . . . . . . . . . . . . . 94

2.9 On-line Data Acquisition System . . . . . . . . . . . 94

2.10 Off-line Crystal Calibration $\ldots \ldots \ldots \ldots \ldots \ldots$. . . . 97

2.11 Crystal Ball Jargon $\ldots \ldots \ldots \ldots \ldots \ldots$

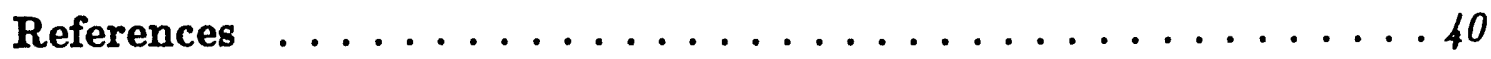


3. DATA PROCESSING $\ldots \ldots \ldots \ldots \ldots \ldots \ldots \ldots \ldots \ldots$

3.1 Off-line Analysis $\ldots \ldots \ldots \ldots \ldots \ldots \ldots \ldots$

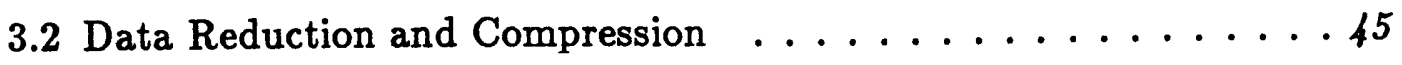

3.3 Additional Data Processing $\ldots \ldots \ldots \ldots \ldots \ldots$

3.4 Hadron Selection $\ldots \ldots \ldots \ldots \ldots \ldots \ldots \ldots \ldots \ldots \ldots$

3.5 Monte Carlo Simulations $\ldots \ldots \ldots \ldots \ldots \ldots \ldots$

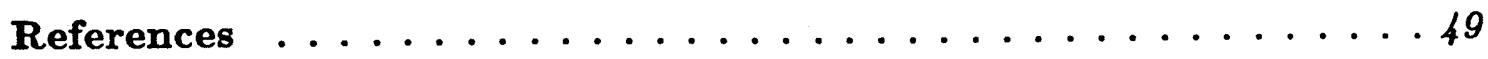

4. THE $\Upsilon$ (1S) PHOTON SPECTRUM ............ . . . 50

4.1 The $\Upsilon(1 \mathrm{~S})$ Data Set . . . . . . . . . . . . 50

4.2 Determining Cuts Which Optimize Sensitivity $\ldots \ldots \ldots \ldots 5$

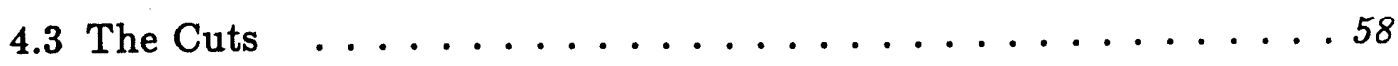

4.3.1 Charge/Neutral Separation . . . . . . . . . . 58

4.3.2 Photon Pattern Cuts . . . . . . . . . . . .62

4.3.3 Particle Overlap Cut . . . . . . . . . . . 71

4.3.4 Low-Energy $\pi^{0}$ Subtraction . . . . . . . . . . . 71

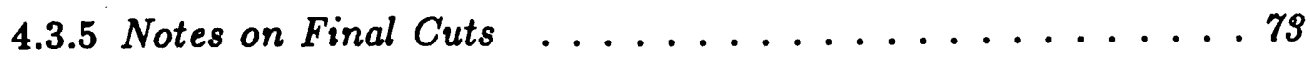

4.4 The $\Upsilon(1 \mathrm{~S})$ Photon Spectrum $\ldots \ldots \ldots \ldots \ldots \ldots$

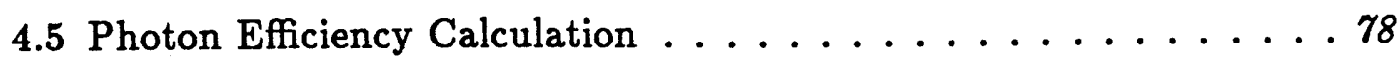

4.6 Results . . . . . . . . . . . . . . 80

4.7 Conclusions $\ldots \ldots \ldots \ldots \ldots \ldots \ldots$

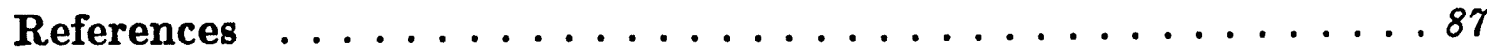

Appendix A. Problems in the Crystal Energy Distributions . . . 88

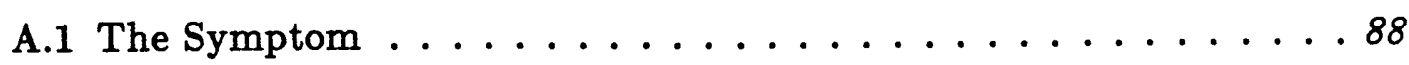

A.2 The Problem Found . . . . . . . . . . . . 91

A.3 Measurement of the Non-linear Response . . . . . . . . . 100

A.4 Modeling the Problem . . . . . . . . . . . . . 109

A.5 Results of CIS Measurements . . . . . . . . . . . 107

Appendix B. Information Useful for Combining Data Sets . . 112

B.1 Information Useful for Combining Data Sets . . . . . . . 112 
[1.1] The effective potential after radiative corrections in the stan-

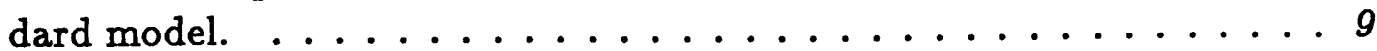

[1.2] Feynman diagram for Higgs production via $Z^{0}$

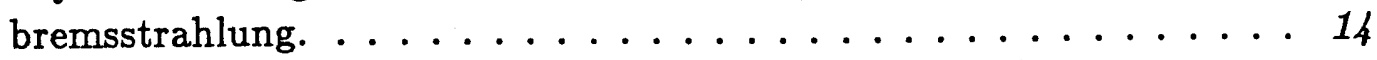

[1.3] Feynman diagram for the Wilczek mechanism. . . . . . . 16

[1.4] The branching ratio for $\Upsilon \rightarrow \gamma+H$ predicted by the Wilczek

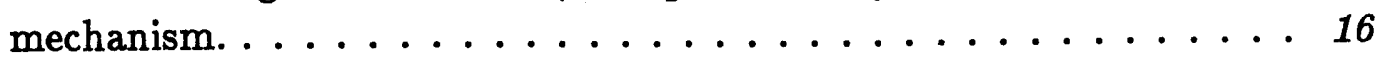

[1.5] The final $\Upsilon(1 \mathrm{~S})$ inclusive photon spectrum. . . . . . . . . . 17

[1.6] The 90\% confidence level upper limit for the process $\Upsilon(1 \mathrm{~S}) \rightarrow$

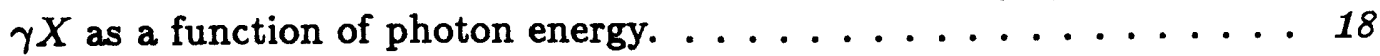

[1.7] The $90 \%$ confidence level upper limit for the process $\Upsilon(1 \mathrm{~S}) \rightarrow$ $\gamma X$ as a function of recoil mass. . . . . . . . . . . 19

[1.8] The upper limit for $B R(\Upsilon \rightarrow \gamma X)$ as a function of photon

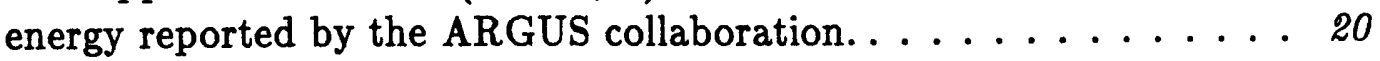

[1.9] The upper limit for $B R(\Upsilon \rightarrow \gamma X)$ as a function of photon energy reported by the CLEO collaboration. . . . . . . . 21

[1.10] The upper limit for $B R(\Upsilon \rightarrow \gamma X)$ as a function of recoil mass squared reported by the CUSB collaboration. . . . . . . . 22

[2.1] Layout of the DORIS II storage ring. . . . . . . . . . . . 26

[2.2] The major components of the Crystal Ball at DORIS II. . . . . . 27

[2.3] The underlying structure of the Crystal Ball. . . . . . . . . . 29

[2.4] The Crystal Ball tube chambers. . . . . . . . . . . . . 22

[2.5] The Crystal Ball on-line data acquisition system. . . . . . . . 95

[2.6] A close-up projection of the Crystal Ball for a typical EM

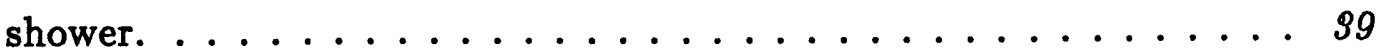


[3.1] Definition of Connected Regions and Bumps. . . . . . . . 49

[4.1] The integrated luminosity collected on the $\Upsilon(1 \mathrm{~S})$ by the Crystal Ball. . . . . . . . . . . . . . . . 51

[4.2] The number of selected hadrons per selected Bhabha. . . . . . . . 52

[4.3] A peak in an arbitrary spectrum. . . . . . . . . . . 57

[4.4] The invariant mass plot for neutral-neutral combinations. . . . . . 660

[4.5] The result of minimizing $R$ for the variable $E 1 / E 4$ shown for 3 different photon energies. . . . . . . . . . 6 69

[4.6] The results from minimizing $R$ for $E 1 / E 4$ for all 10 photon

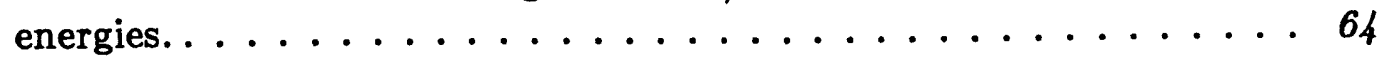

[4.7] The result of minimizing $R$ for $E 1 / E 4$ with photon energy of

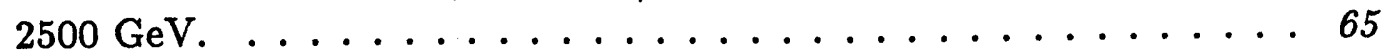

[4.8] The results from minimizing $R$ for $E 4 / E 13 . \ldots \ldots \ldots \ldots 6$

[4.9] The result of minimizing $R$ for the variable $E 2 / E 4$ shown for 3 different photon energies. . . . . . . . . . . 67

[4.10] The result of minimizing $R$ for the variable $E 13 / E_{C R}$ shown

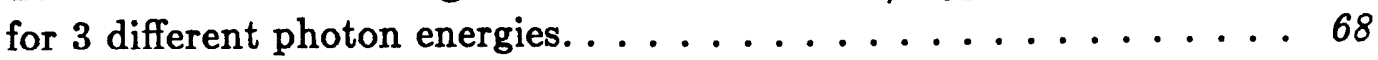

[4.11] The results from minimizing LLD from PIFIT for 9 photon energies. . . . . . . . . . . . . . 69

[4.12] The result of minimizing $R$ for LLD from PIFIT shown for 3

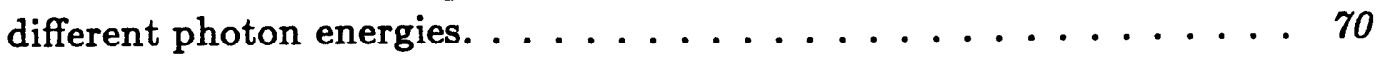

[4.13] The results of minimizing $R$ for the minimum opening angle

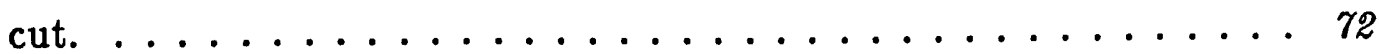

[4.14] The inclusive photon spectrum after each cut in the analy-

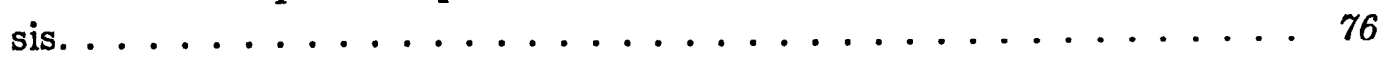

[4.15] The final $\Upsilon(1 \mathrm{~S})$ inclusive photon spectrum. . . . . . . . . T7

[4.16] The final $\Upsilon(1 S)$ inclusive photon spectrum plotted as a function of $x=E_{\gamma} / E_{\text {beam }} \ldots \ldots \ldots \ldots \ldots \ldots \ldots \ldots \ldots$

[4.17] The photon efficiency for the process $\Upsilon(1 S) \rightarrow \gamma X$ as a function of photon energy. . . . . . . . . . . 81

[4.18] The statistical significance, $S / \Delta S$, of any structure consistent with a narrow state in the inclusive photon spectrum. . . . . . 82

[4.19] The $90 \%$ confidence level upper limit for the process $\Upsilon(1 \mathrm{~S}) \rightarrow$ $\gamma X$ as a function of photon energy. . . . . . . . . 84 
[4.20] The $90 \%$ confidence level upper limit for the process $\Upsilon(1 \mathrm{~S}) \rightarrow$ $\gamma X$ as a function of recoil mass. . . . . . . . . . . . . . 85

[A.1] Crystal energy distributions in the 1984 data. . . . . . . . . . 89

[A.2] The basic setup for the Crystal Ball on-line system. . . . . . . . 91

[A.3] The voltage level for the Analog-Out scan. . . . . . . . . . 99

[A.4] One Channel in the Analog-Out scan. . . . . . . . . . 95

[A.5] The output schematic for the INH output circuit. . . . . . . . 96

[A.6] The effects of different op-amps on the settling time. .......97

[A.7] The effects of different FET switches on the settling time. . . . . . 98

[A.8] The electronics setup for the charge injection system. . . . . . . 100

[A.9] Comparison of predicted and measured crystal energy distributions for crystal 434. . . . . . . . . . . . . . 104

[A.10] Comparison of predicted and measured crystal energy distributions for crystal 156. . . . . . . . . . . . . 105

[A.11] Comparison of predicted and measured crystal energy distributions for crystal 143. . . . . . . . . . . . . . 106

[A.12] The change in the mean and sigma as modeled by CISSY. . . . . . 109

[A.13] The photon efficiency as a function of energy for the 1984

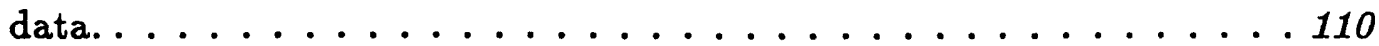




\section{List of Tables}

[2.1] A list of dimensions and Radiation Lengths for the Crystal Ball tracking chambers. .......................... 44

[2.2] The nominal values of constants used in the crystal energy

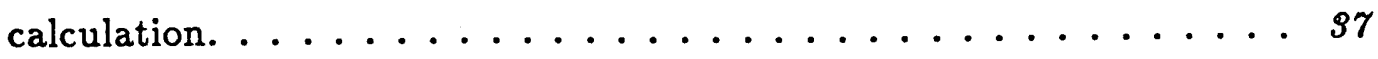

[3.1] The major off-line production steps. . . . . . . . . . 42

[4.1] Numerical quantities used in the calculation of produced

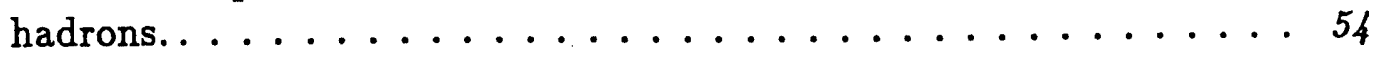

[4.2] Initial cuts used to define the $\Upsilon(1 \mathrm{~S})$ data set. . . . . . . . . . 59

[4.3] The results of several possible charge criteria on the minimization of $R . \ldots \ldots \ldots \ldots 1$

[4.4] The final set of cuts used in this analysis. . . . . . . . . 74

[A.1] The electronics' hardware configuration for the 1983 and 1984

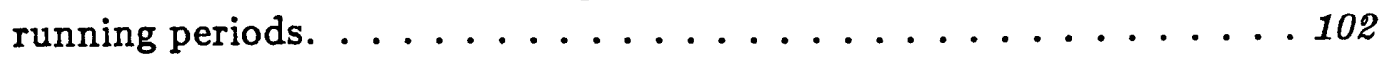

[A.2] The cuts used to define photon in the efficiency calcula-

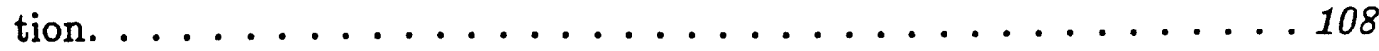

[B.1] The numerical quantities used in the upper limit calculation as a function of photon energy. ...................... 


\section{INTRODUCTION AND SUMMARY}

\subsection{The Standard Model}

Over the last 30 years, particle physicists have made impressive gains in understanding matter and its interactions involved in all phenomena observed to date. The fundamental concept driving this revelation is that of symmetry. This is most transparent in Lagrangian field theory, where each global symmetry of the Lagrangian implies a conservation law by Noether's theorem. Experimentally established conservation laws can then restrict the mathematical form of the Lagrangian. An example of this is gauge invariance which places a powerful constraint on the form of the Lagrangian. The freedom to choose a particular gauge leads at once to the existence of conserved charges and currents. If one goes further and requires the Lagrangian to be locally gauge invariant, one is required to add additional fields in the form of massless gauge bosons which can be interpreted as particles mediating interactions. Quantum Electrodynamics (QED) is the premier example, where the massless gauge boson is associated with the photon. When the Lagrangian is invariant under some symmetry which is not respected by the ground state, the symmetry is said to be spontaneously broken. If this symmetry is an exact continuous one, spontaneous symmetry breaking will cause massless spin-zero particles, called Goldstone bosons, to appear.

It might seem that the use of local gauge invariance is restrictive because it 
implies a massless vector boson will mediate interactions, when the photon is the only such particle known. If in addition, the gauge symmetry is spontaneously broken, one might expect a massless spin-zero Goldstone boson to appear; no such particles are known to exist. However, when a local gauge symmetry is spontaneously broken a remarkable interaction takes place; the Goldstone boson fails to appear in the particle spectrum while the gauge boson acquires this extra degree of freedom, giving it mass. The two unwanted massless particles combine to give the desired massive gauge boson by what is called the Higgs mechanism [ ${ }^{[1]}$ The one undesirable aspect of this synthesis is that an undetected massive scalar particle, called the Higgs boson, is predicted to exist. Because there exists no evidence for this particle, attempts have been made to develop a theory which has the desirable qualities of a spontaneously broken gauge theory while keeping a fundamental Higgs boson out of the particle spectrum. Usually these attempts take the form of dynamical symmetry breaking where the symmetry is broken by scalar bound states, arising from radiative corrections, which play the role of the Higgs boson. Here the dynamics of the system itself break the symmetry. Unfortunately, no completely satisfactory approach along these lines has appeared thus far.

Several authors, notably S. L. Glashow, S. Weinberg and A. Salam, ${ }^{[2]}$ contributed to what is now called the "Standard Model" by imposing the type of symmetry arguments discussed above to form a unified theory of the weak and electromagnetic interactions. The standard model is a generalization of this electroweak theory which includes the $S U(3)_{C}$ group of colored quarks. Because the primary features of the Higgs mechanism do not depend on the inclusion of quarks, only the electroweak theory will be discussed below. In the minimal electroweak theory, the fundamental fermions form left-handed weak-isospin doublets

$$
L_{e}=\left(\begin{array}{c}
\nu_{e} \\
e
\end{array}\right)_{L} \quad L_{\mu}=\left(\begin{array}{c}
\nu_{\mu} \\
\mu
\end{array}\right)_{L} \quad L_{\tau}=\left(\begin{array}{c}
\nu_{\tau} \\
\tau
\end{array}\right)_{L}
$$


where the left-handed projections are

$$
\begin{aligned}
& \nu_{e L}=\frac{1}{2}\left(1-\gamma_{5}\right) \nu_{e} \quad \nu_{\mu L}=\frac{1}{2}\left(1-\gamma_{5}\right) \nu_{\mu} \quad \nu_{\tau L}=\frac{1}{2}\left(1-\gamma_{5}\right) \nu_{\tau} \\
& e_{L}=\frac{1}{2}\left(1-\gamma_{5}\right) e \quad \mu_{L}=\frac{1}{2}\left(1-\gamma_{5}\right) \mu \quad \tau_{L}=\frac{1}{2}\left(1-\gamma_{5}\right) \tau .
\end{aligned}
$$

This doublet structure allows the weak charged current to contain left-handed spinors. Because right-handed weak charged currents are not observed in nature, the right handed fermions form weak-isospin singlets

$$
R_{e}=e_{R} \quad R_{\mu}=\mu_{R} \quad R_{\tau}=\tau_{R}
$$

where the right-handed projections are

$$
e_{R}=\frac{1}{2}\left(1+\gamma_{5}\right) e \quad \mu_{R}=\frac{1}{2}\left(1+\gamma_{5}\right) \mu \quad \tau_{R}=\frac{1}{2}\left(1+\gamma_{5}\right) \tau
$$

For neutrinos,

$$
\nu_{R}=\frac{1}{2}\left(1+\gamma_{5}\right) \nu=0
$$

for each of the three generations. This condition implies that the neutrino will not get a mass from the standard lepton-Higgs coupling. However, neutrinos can obtain a mass through a Majorana mass term in the Lagrangian of the form

$$
\Delta L=m \bar{\nu}^{c} \nu
$$

In the Standard model, this term is $\Delta I=1$ and therefore very small.

Electromagnetism is incorporated by constructing a weak hypercharge, $\mathrm{Y}$, which commutes with the weak isospin and satisfies the Gell-Mann-Nishijima relation

$$
Q=I_{3}+\frac{1}{2} Y
$$


The gauge group is then $S U(2)_{L} \otimes U(1)_{Y}$. A Lagrangian is constructed which is invariant under this group by defining a field $A_{\mu}$, with charge $g^{\prime} / 2$, for the $U(1)$ group and a triplet of gauge fields $B_{\mu}^{i}$, with charge $g$, for the $S U(2)_{L}$ group:

$\begin{array}{lll}A_{\mu} & U(1)_{Y} & g^{\prime} / 2 \\ B_{\mu}^{1}, B_{\mu}^{2}, B_{\mu}^{3} & S U(2)_{L} & g\end{array}$

Invoking spontaneous symmetry breaking gives mass to three of the gauge fields while keeping the other field massless. This later field will be associated with the photon. Spontaneous symmetry breaking will also permit mass terms for the electron, muon and tau, which would normally be absent since they would violate the $S U(2)$ invariance. The $S U(2)_{L} \otimes U(1)_{Y}$ group is most easily broken by introducing a doublet of complex scalar fields

$$
\phi=\left(\begin{array}{l}
\phi^{+} \\
\phi^{0}
\end{array}\right)
$$

and the most general, stable, renormalizable potential

$$
V\left(\phi^{\dagger} \phi\right)=\mu^{2} \phi^{\dagger} \phi+|\lambda|\left(\phi^{\dagger} \phi\right)^{2}
$$

When $\mu^{2}<0$, the field $\phi$ acquires a non-zero vacuum expectation value, which can be chosen real:

$$
\langle\phi\rangle_{0}=\frac{1}{\sqrt{2}}\left(\begin{array}{l}
0 \\
v
\end{array}\right)
$$

where $v=\sqrt{-\mu^{2} /|\lambda|}$. This breaks both the $S U(2)_{L}$ and $U(1)_{Y}$ symmetries but the symmetry under $U(1)_{Q}$ is preserved, which is necessary for a massless photon. The Lagrangian can be expanded about $\langle\phi\rangle_{0}$ by defining four new fields $\vec{\zeta}$ and $\eta$ so that

$$
\phi=\exp \left(\frac{i \vec{\zeta} \cdot \vec{\tau}}{2 v}\right)\left(\begin{array}{c}
0 \\
(v+\eta) / \sqrt{2}
\end{array}\right)
$$


where the $\zeta_{i}$ are 3 independent fields and the $\tau_{i}$ are the Pauli spin matrices. By transforming the Lagrangian to the unitary gauge where the physical states appear explicitly, it is straightforward to define new gauge fields

$$
\begin{aligned}
W_{\mu}^{ \pm} & =\frac{\left(B_{\mu}^{1} \mp i B_{\mu}^{2}\right)}{\sqrt{2}} \\
Z_{\mu} & =\frac{-g B_{\mu}^{3}+g^{\prime} A_{\mu}}{\sqrt{g^{2}+g^{\prime 2}}} \\
A_{\mu} & =\frac{-g^{\prime} B_{\mu}^{3}+g A_{\mu}}{\sqrt{g^{2}+g^{\prime 2}}}
\end{aligned}
$$

The $A_{\mu}$ field remains massless, while the $W_{\mu}^{ \pm}$and $Z_{\mu}$ fields acquire mass terms:

$$
\begin{aligned}
M_{W \pm} & =g v / 2 \\
M_{Z^{0}} & =\frac{v}{2} \sqrt{g^{2}+g^{\prime 2}}=M_{W \pm} \sqrt{1+g^{\prime 2} / g^{2}}
\end{aligned}
$$

In this gauge, the $\zeta$ field disappears completely from the Lagrangian. The $\zeta$ 's three degrees of freedom were not lost, nor did they appear as Goldstone bosons, but rather were absorbed into an additional helicity state for the three gauge bosons, the $W^{ \pm}$and $Z^{0}$, giving them mass. The $\eta$ field, on the other hand, acquires a mass term and corresponds to the Higgs boson with mass

$$
M_{H}^{2}=-2 \mu^{2}>0
$$

which is both positive and arbitrary.

If this model is compared to other models of the weak interaction which correctly describe the low energy behavior (but fail at higher energies), then an association can be made with the Fermi's coupling constant $G_{F}$. By comparing terms in the Lagrangians one finds

$$
g^{2} / 8=G_{F} M_{W}^{2} / \sqrt{2}
$$


which implies

$$
\begin{aligned}
v & =\left(G_{F} \sqrt{2}\right)^{-\frac{1}{2}} \simeq 246 \mathrm{GeV} \\
\langle\phi\rangle_{0} & =\left(G_{F} \sqrt{8}\right)^{-\frac{1}{2}} \simeq 174 \mathrm{GeV}
\end{aligned}
$$

Similarly, the electromagnetic charge $e$ can be related to the coupling constants $g$ and $g^{\prime}$ :

$$
e=g g^{\prime} / \sqrt{g^{2}+{g^{\prime}}^{2}}=g^{\prime} \cos \theta_{W}=g \sin \theta_{W}
$$

where $\theta_{W}$ is used to parametrize the mixing of the $B_{\mu}^{3}$ and $A_{\mu}$ fields. Mass relations for the $W^{ \pm}$and $Z^{0}$ can now be written in terms of $\theta_{W}$. To lowest order these $\operatorname{are}^{[3]}$

$$
\begin{aligned}
M_{W}^{2} & =g^{2} / 4 G_{F} \sqrt{2} \\
& =\pi \alpha / G_{F} \sqrt{2} \sin ^{2} \theta_{W} \\
& \simeq\left(37.28 \mathrm{GeV} / \mathrm{c}^{2}\right)^{2} / \sin ^{2} \theta_{W} \\
M_{Z}^{2} & =M_{W}^{2} / \cos ^{2} \theta_{W}
\end{aligned}
$$

Radiative corrections modify these relations so that

$$
M_{W}^{2}=\pi \alpha / G_{F} \sqrt{2}(1-\Delta r) \sin ^{2} \theta_{W}
$$

where the $\Delta r$ contribution arises primarily from $\alpha$ evolving from $\alpha^{-1} \simeq 137.0$ at $Q^{2}=0$, to $\alpha^{-1} \simeq 127.5$ at $Q^{2}=M_{W}$. This $\Delta r$ correction has been calculated ${ }^{[4]}$ to be about $\Delta r=0.036$. The more accurate equation now reads

$$
\sin ^{2} \theta_{W} \simeq \frac{\left(38.5 \mathrm{GeV} / c^{2}\right)^{2}}{M_{W}^{2}}
$$

Before 1983, neutrino-electron scattering experiments, studying charged and neutral current interaction, indicated ${ }^{[s]}$

$$
\sin ^{2} \theta_{W} \simeq 0.216 \pm 0.010 \pm 0.004
$$


implying the $W^{ \pm}$and $Z^{0}$ masses were

$$
M_{W} \simeq 83.0_{-2.8}^{+3.0} \mathrm{GeV} / c^{2} \quad M_{Z} \simeq 93.8_{-2.4}^{+2.5} \mathrm{GeV} / \mathrm{c}^{2}
$$

In 1983, the UA1 and UA2 collaborations, running at the CERN SPS $\bar{p}$ p collider presented the first direct evidence for the existence of the $W^{ \pm}$and $Z^{0}$. The measured values were ${ }^{[6]}$

$$
\begin{aligned}
M_{W} & =(80.9 \pm 1.5 \pm 2.4) \mathrm{GeV} / \mathrm{c}^{2} & M_{W} & =(81.0 \pm 2.5 \pm 1.3) \mathrm{GeV} / \mathrm{c}^{2} \\
M_{Z} & =(95.6 \pm 1.4 \pm 2.9) \mathrm{GeV} / \mathrm{c}^{2} & M_{Z} & =(91.9 \pm 1.3 \pm 1.4) \mathrm{GeV} / \mathrm{c}^{2}
\end{aligned}
$$

for the two groups respectively. From such measurements one can calculate $\sin ^{2} \theta_{W}$ from formula [1.2]. Using a weighted average of UA1 and UA2's most recent results one gets $^{[7]}$

$$
\sin ^{2} \theta_{W}=0.223 \pm 0.008 \quad \text { (UA1 and UA2). }
$$

Averaging the value of $\sin ^{2} \theta_{W}$ obtained from many experiments, including the UA1 and UA2 results, several neutrino-lepton and neutrino-nucleon scattering experiments, forward-backward asymmetry measurements from $e^{+} e^{-} \rightarrow \mu^{+} \mu^{-}$, and atomic parity violation results yields ${ }^{[8]}$

$$
\sin ^{2} \theta_{W}=0.223 \pm 0.004 \quad \text { (world average) }
$$

The prediction and subsequent discovery of the $W^{ \pm}$and $Z^{0}$ gauge bosons, among many other triumphs, has given physicists confidence that the standard model is correct to a good level of approximation. Indeed, no experimental data are in conflict with this theory at present. However, the standard model falls short in several respects; is does not explain the origin of the fermion generations, the quark and lepton masses, the mixing between quark flavors, CP violation, why the weak charged-current interactions are left handed, and other fundamental questions. In the very likely event that new physics is discovered at higher energies, any comprehensive model will most likely have the standard model as its low-energy approximation. 


\subsection{The Higgs Sector}

Although the predictive power of the standard model is impressive, it says very little about the Higgs boson. The standard Higgs is a neutral scalar which couples to all particles in proportion to their mass. The coupling to any particle $\mathrm{X}$ is given by

$$
G_{\mathrm{X} \overline{\mathrm{X}} \mathrm{H}}=\frac{2 M_{\mathrm{X}}}{v} \simeq \frac{M_{\mathrm{X}}}{123 \mathrm{GeV} / \mathrm{c}^{2}}
$$

where $M_{\mathrm{X}}$ is measured in $\mathrm{GeV} / \mathrm{c}^{2}$.

The most interesting quantity, in relation to experimental searches, is it's mass. Because experimental detection of the standard Higgs is difficult, as will be shown below, its discovery would be made much easier if a more definitive statement could be made regarding its mass. By applying internal consistency arguments, one can at least make upper and lower bounds on its mass.[10] Taking into account quantum corrections, one can calculate the effective potential for the scalar $\phi$ fields, corresponding to equation [1.1]. The effective potential can be approximated by summing tree-level and one-loop diagrams with the assumption that all fermions are much lighter than the $W^{ \pm}$and $Z^{0}$. The form of the effective potential in this case is shown in Figure 1.1 and is ${ }^{[1]}$

$$
V_{\mathrm{eff}}\left(\phi^{2}\right)=\mu^{2} \phi^{2}+B \phi^{4} \ln \left(\phi^{2} / M_{f}^{2}\right)
$$

where $B$ is a positive constant

$$
B=\left(\frac{3}{64 \pi^{2}\langle\phi\rangle_{0}^{4}}\right) \sum_{v} M_{v}^{4}
$$

where the sum runs over the vector gauge bosons and $M_{f}$ is an arbitrary mass parameter. If $T \equiv 2 B\langle\phi\rangle_{0}^{2} / M_{H}^{2}$ is between 0 and $\frac{1}{2}, V(\phi)$ will have one minimum away from the origin. For $T$ between $\frac{1}{2}$ and $1, V$ will have an absolute minimum away from the origin and a local minimum at $\phi=0$. If $T$ is greater than 1 , the 


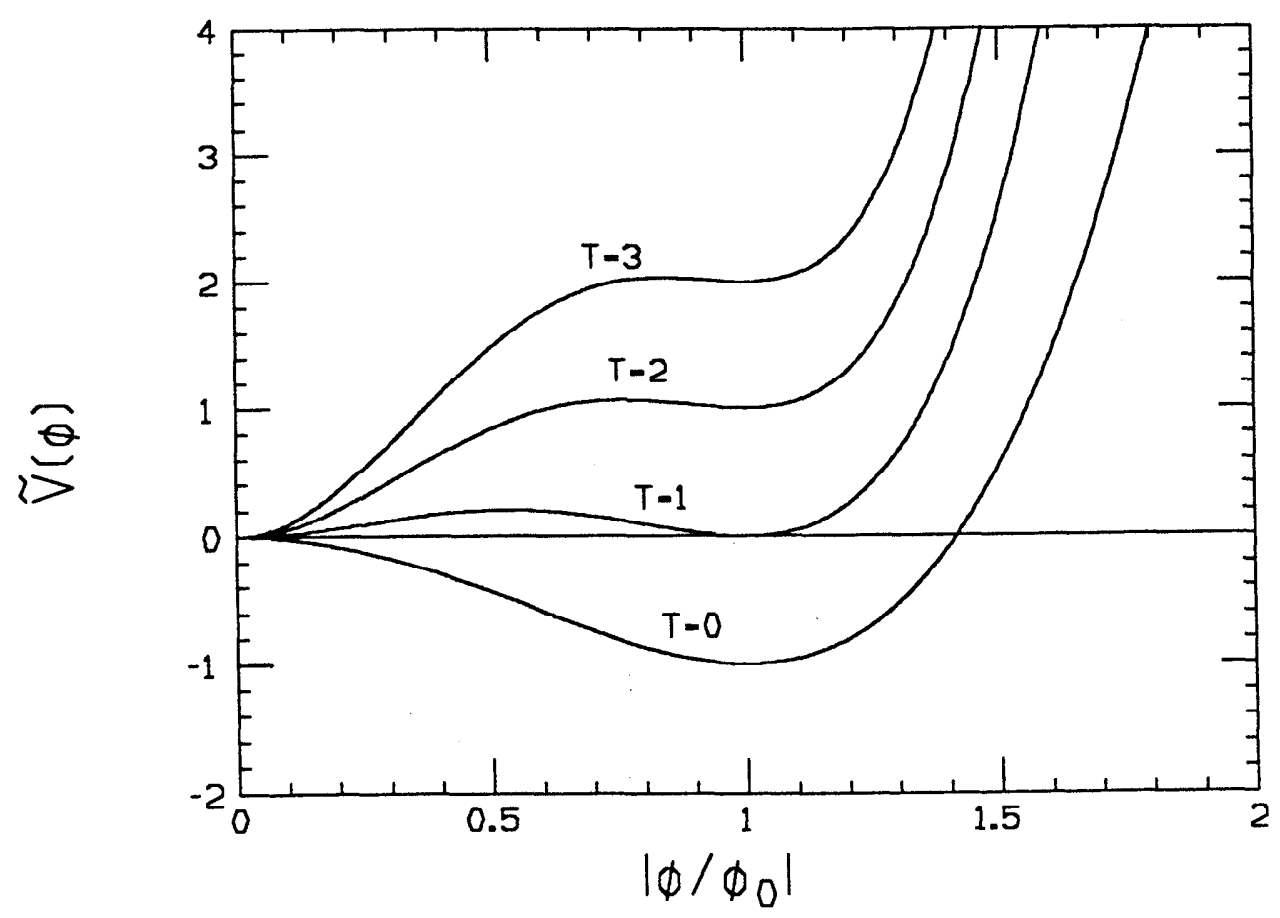

Fig. 1.1 The effective potential after radiative corrections in the standard model. The scaled potential $\tilde{V}(\phi) \equiv 4 V(\phi) / M_{H}^{2} \phi_{0}^{2}$ is plotted. Depending on $T$ (defined in the text) the minimum away from the origin can be stable, metastable, or unstable.

absolute minimum will be at $\phi=0$ and a local minimum will exist away from the origin. The requirement that the minimum away from the origin, which is responsible for symmetry breaking, is absolute $(T \leq 1)$, leads to the lower limit

$$
M_{H}^{2} \geq \frac{3 \sqrt{2} G_{F}}{16 \pi^{2}}\left(2 M_{W}^{4}+M_{Z}^{4}\right)=\frac{3 \alpha^{2}\left(2+\sec ^{4} \theta_{W}\right)}{16 \sqrt{2} G_{F} \sin ^{4} \theta_{W}}
$$

called the Weinberg-Linde limit. For $\sin ^{2} \theta_{W}=.223$ this relation implies $M_{H} \geq$ $6.7 \mathrm{GeV} / \mathrm{c}^{2}$. If two-loop diagrams are taken into account, this lower limit must be raised by about $300 \mathrm{MeV}^{[12]}$ If one assumes the effective potential's minimum away from the origin is not an absolute minimum, but corresponds to a long-lived metastable vacuum, the probability that a local quantum fluctuation could cause the vacuum state to tunnel to the absolute minimum can be calculated! ${ }^{[3]}$ The lower limit, assuming this tunneling is not probable over the age of the universe, 
is about $10.4 \mathrm{GeV} / \mathrm{c}^{2}$.

A lower limit can be formulated which does not assume all fermions are lighter than the vector gauge bosons. Using the branching ratios for $K^{ \pm} \rightarrow \pi^{ \pm} e^{+} e^{-}$and $K^{ \pm} \rightarrow \pi^{ \pm} \mu^{+} \mu^{-}$, the lower limit

$$
M_{H} \geq 325 \mathrm{MeV} / \mathrm{c}^{2}
$$

can be derived!14] Many assumptions are made in deriving this upper limit so it may only be approximately correct. Constraints from macroscopic, atomic and nuclear physics require $M_{H}>O\left(15 \mathrm{MeV} / \mathrm{c}^{2}\right)^{[15]}$ with more confidence.

An upper limit on the Higgs boson mass is obtained by considering $W^{+} W^{-} \rightarrow$ $W^{+} W^{-}$scattering for longitudinally polarized $W^{\prime}$ s. At high energy, the amplitude scales like

$$
T \sim\left(-4 G_{F} / \sqrt{2}\right) M_{H}^{2}, \quad s \rightarrow \infty
$$

When the partial-wave unitarity bound, $\left|T^{J}\right| \leq 1$, is applied to the S-wave amplitude, where

$$
T=16 \pi \sum_{J}(2 J+1) T^{J} P_{J}(\cos \theta)
$$

one finds

$$
M_{H}^{2} \leq 4 \pi \sqrt{2} / G_{F}=\left(1.2 \mathrm{TeV} / \mathrm{c}^{2}\right)^{2}
$$

If various assumptions are used to further constrain the Higgs sector of the standard model, some definite predictions on the Higgs mass can be made. If one assumes a linear relationship between the quartic scalar coupling, $\lambda$, and the squares of the gauge couplings, $g$ and $g^{\prime}$,

$$
\lambda=c_{1} g^{2}+c_{2} g^{\prime 2},
$$


the mass of the Higgs is uniquely determined ${ }^{[16]}$ to be $49 \pm 2 \mathrm{GeV} / \mathrm{c}^{2}$. A very different prediction arises from the assumption that at high energies, scattering of longitudinally polarized $\mathrm{W}$ bosons is softened by some unspecified strong interaction in the $t$-channel. If the amplitude vanishes sufficiently fast at high energies, the prediction

$$
M_{H} \simeq 2.3 M_{W} \simeq 190 \mathrm{GeV} / \mathrm{c}^{2}
$$

can be made ${ }^{[7]}$ Although the assumptions which give rise to these predictions have little or no experimental justification, the results are presented here to indicate the large theoretical uncertainty in the Higgs sector.

A much lower Higgs mass is predicted if one drops the mass term in the scalar potential by setting $\mu^{2}=0$ in equation [1.1] above. In this case, radiative corrections to the effective potential break the symmetry dynamically! ${ }^{[8]}$ The Higgs mass is then determined to be

$$
M_{H}^{2}=\frac{3 \alpha^{2}}{8 \sqrt{2} G_{F}}\left\{\frac{2+\sec ^{4} \theta_{W}}{\sin ^{4} \theta_{W}}-O\left(\frac{M_{f}^{4}}{M_{W}^{4}}\right)\right\}
$$

If all fermions masses, $M_{f}$, are much less than the $\mathrm{W}$ boson's, $\sin ^{2} \theta_{W}=.223$ results in $M_{H} \simeq 9.4 \mathrm{GeV} / \mathrm{c}^{2}$. Again, two-loop corrections ${ }^{[12]}$ raise this number to about $10.4 \mathrm{GeV} / \mathrm{c}^{2}$. This prediction is interesting because it is experimentally accessible and this mass is within the $\Upsilon$ system where mixing could enhance the Higgs boson production.

The standard model is described by three fundamental parameters, excluding fermion masses. Two of these parameter, $\alpha=1 / 137.036$ and $G_{F}=1.16637 \times$ $10^{-5} \mathrm{GeV}^{-2}$, are determined quite accurately. The third fundamental parameter can be taken as either $\sin ^{2} \theta_{W}, M_{W}, M_{Z}$ or some combination of these, where the remaining two quantities are then uniquely determined. Since the mass of the $W^{ \pm}$and the $Z^{0}$ are now directly measurable, the ratio $M_{W} / M_{Z}$ is usually taken 
to be the third parameter. In this way, the $\rho$ parameter, defined as

$$
\rho=\frac{M_{W}^{2}}{M_{Z}^{2} \cos ^{2} \theta_{W}} \equiv 1
$$

can be set to one in the standard model, independent of radiative corrections. The measured values of $M_{W}$ and $M_{Z}$ from UA1 and UA2, and $\sin ^{2} \theta_{W}$ from other experiments, produces the measurement ${ }^{[8]}$

$$
\rho=1.006 \pm 0.008
$$

in agreement with the standard model. This measurement is important for attempts to formulate more complicated models of the Higgs sector than just one Higgs doublet. Grand unified theories and theories where CP-violation occurs directly by spontaneous symmetry breaking, for example, involve such extensions. These models are constrained to have only weak isospin doublets because larger multiplets imply $\rho \neq 1$ in general, unless the vacuum expectation values are carefully chosen; with only weak isospin doublets, $\rho=1$ is assured. In the simplest extension of the standard model, two Higgs doublets are used to break the symmetry. In this case, five Higgs bosons appear; three are electrically neutral and two charged. Models such as this are popular because charged Higgs would be much easier to detect experimentally. These theories often have more freedom than the minimal model so the Higgs masses are generally even less constrained.

In summary, the Higgs boson mass is theoretically quite unconstrained. In the minimal Higgs sector, it is somewhere between about $7 \mathrm{GeV} / \mathrm{c}^{2}$ and $1 \mathrm{TeV} / \mathrm{c}^{2}$, with assumptions about the fermion masses. Without assumptions on the fermion masses the lower limit is $O\left(100 \mathrm{MeV} / \mathrm{c}^{2}\right)$. Experimentally, no Higgs mass has been excluded in this energy range. 


\subsection{Searching for the Higgs Boson in Radiative Upsilon Decays}

Understanding the Higgs sector has been called "Problem Number One" in particle physics $!^{[19]}$ Because the Higgs sector is not well understood theoretically and because of its central importance in symmetry breaking and mass generation, experimental results are desperately needed to constrain models. These results will most likely come from experiments running at $\mathrm{p} \overline{\mathrm{p}}$ colliders and/or $e^{+} e^{-}$ machines.

The most promising Higgs boson production mechanisms at $p \bar{p}$ colliders are expected to be very difficult to observe. Gluon fusion has a very low cross section

$$
\sigma(\mathrm{p} \overline{\mathrm{p}} \rightarrow H+X) \simeq(1 \text { to } 100) \mathrm{pb}
$$

for a Higgs mass ranging from about $100 \mathrm{GeV} / \mathrm{c}^{2}$ down to about $5 \mathrm{GeV} / \mathrm{c}^{2}$, respectively for $\sqrt{s} \approx(400$ to 2000$) \mathrm{GeV}$. Not only is this cross section too small for Higgs detection at present, but its decay will mimic that of heavy quarks. Another possibility, quark bremsstrahlung, may have a better experimental signature. If the heavy quarks are charm quarks and the Higgs decays into $\tau$ pairs, the final state $\mathrm{p} \overline{\mathrm{p}} \rightarrow \mathrm{c} \overline{\mathrm{c}} \tau \bar{\tau} X$ should be distinctive, however the cross section is again small.

Higgs production in $e^{+} e^{-}$machines is also difficult because its coupling to any particle is proportional to its mass. The continuum production of Higgs bosons is reviewed in Reference 21. Calculations show the cross sections for the processes
A)
$e^{+} e^{-} \rightarrow H \rightarrow$ hadrons
(direct production)
B)
$e^{+} e^{-} \rightarrow \gamma H$
C)
$e^{+} e^{-} \rightarrow \mathrm{q} \overline{\mathrm{q}} H$
(quark Bremsstrahlung)
D)
$e^{+} e^{-} \rightarrow e^{+} e^{-} H$
(2-photon production) 


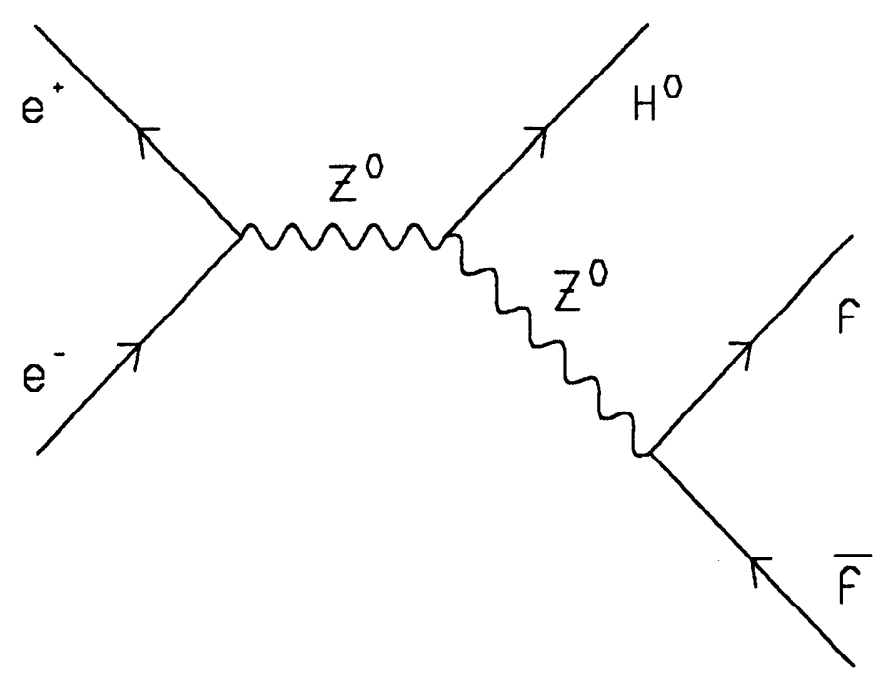

Fig. 1.2 Feynman diagram for Higgs production via $Z^{0}$ bremsstrahlung.

are extremely small. The cross sections for processes $\mathbf{A}$ and $\mathrm{B}$, given in terms of $R=\sigma_{\mathrm{had}} / \sigma_{\mu \mu}$, are about

$$
\begin{aligned}
& \Delta R \approx 2 \times 10^{-3} \\
& \text { ( } e^{+} e^{-} \rightarrow H \rightarrow \text { hadrons) } \\
& \Delta R \approx 1 \times 10^{-6} \\
& \left(e^{+} e^{-} \rightarrow \gamma H\right)
\end{aligned}
$$

for Higgs' mass between about 3 and $30 \mathrm{GeV} / \mathrm{c}^{2}$. Both of these cross sections are far too small to observe at present. Processes $C$ and $D$ also have cross sections that are unobservably small;

$$
\begin{array}{ll}
\sigma \approx 2 \times 10^{-4} \mathrm{pb} & \left(e^{+} e^{-} \rightarrow \mathrm{q} \overline{\mathrm{q}} H\right) \\
\sigma \approx 1 \times 10^{-5} \mathrm{pb} & \left(e^{+} e^{-} \rightarrow e^{+} e^{-} H\right)
\end{array}
$$

Thus, continuum production of the Higgs boson in $e^{+} e^{-}$collisions is not feasible with present experimental techniques.

There is, however, an interesting Higgs production mechanism which may become important as the SLC collider and LEP storage ring become operational. Higgs production by $Z^{0}$ bremsstrahlung, shown in Figure 1.2 could provide a clean signal if the Higgs' mass is near $10 \mathrm{GeV} / \mathrm{c}^{2}[20]$ If the decay fermions of 
the $Z^{0}$ are electron or muon pairs, the event signature should be clean with little background. For a Higgs mass between 10 and $20 \mathrm{GeV} / \mathrm{c}^{2}, B R\left(Z^{0} \rightarrow\right.$ $\left.H^{0} \ell^{+} \ell^{-}\right) \simeq 10^{-4}-10^{-5}$ respectively. With $10^{6} Z^{0}$ decays produced at the SLC or LEP, enough decays of this type could be detected to either find or rule-out a Higgs near $10 \mathrm{GeV} / \mathrm{c}^{2}$; a heavier Higgs, however, requires a rapid increase in sensitivity.

Although continuum production of the Higgs boson is not experimentally possible at this time, heavy quark resonances can provide "outposts" with which to search for the Higgs. The vector resonances, the $\rho, \phi, J / \psi, \Upsilon, \Theta$, and their excited states may provide better opportunities for Higgs production. The Wilczek mechanism ${ }^{[22]}$ by which vector resonances decay to a photon and the Higgs, seems to provide the best possibility for Higgs production to date. Figure 1.3 shows the feynman graph for the process $V \rightarrow \gamma H$. Calculation of this graph yields

$$
\frac{\mathrm{BR}(V \rightarrow \gamma H)}{\mathrm{BR}\left(V \rightarrow \mu^{+} \mu^{-}\right)}=\frac{G_{F} M_{V}^{2}}{4 \sqrt{2} \pi \alpha}\left(1-\frac{M_{H}^{2}}{M_{V}^{2}}\right) .
$$

Because the Higgs couples to quarks in proportion to their mass, the heaviest quark pair experimentally available should be utilized. Radiative decays of the $\Upsilon$ resonances, being the heaviest known quark pairs to date, are thus the best candidates for Higgs production.

The above result, [1.3], must be modified to take into account higher-order corrections. Perturbative QCD radiative corrections ${ }^{[23]}$ are found to lower this estimate by about $\simeq 2$. Mixing between the Higgs and vector $P$-wave states have also been considered $\left[{ }^{24]}\right.$ These effects have been combined taking care to avoid double counting ${ }^{[2]}$ The resulting plot of $\mathrm{BR}(\Upsilon \rightarrow \gamma+H)$ is shown in Figure 1.4. The line labeled (L) is the leading order calculation, while the lines (A) and (B) show the results of two different methods of calculating the first order QCD radiative corrections. It should be noted that because these corrections reduce the rate by about a factor of two, higher-order corrections may further suppress this prediction. 


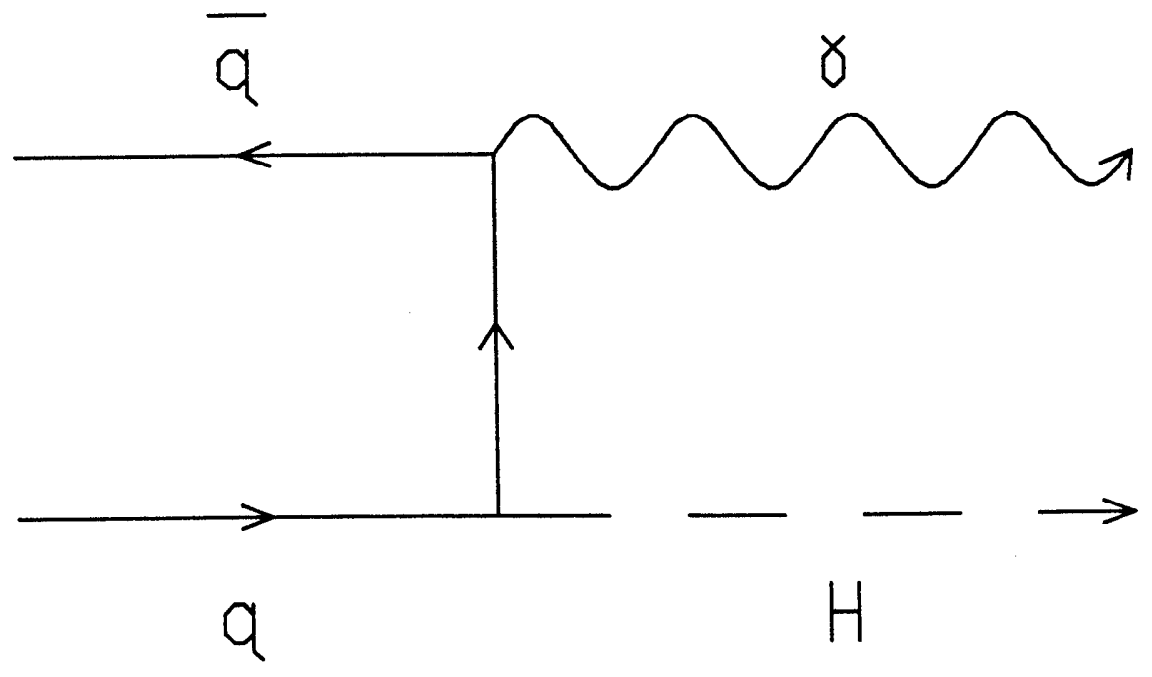

Fig. 1.9 Feynman diagram for the Wilczek mechanism. The leading order process for the $\gamma$ plus Higgs decay of the $q \bar{q}$ vector resonance.

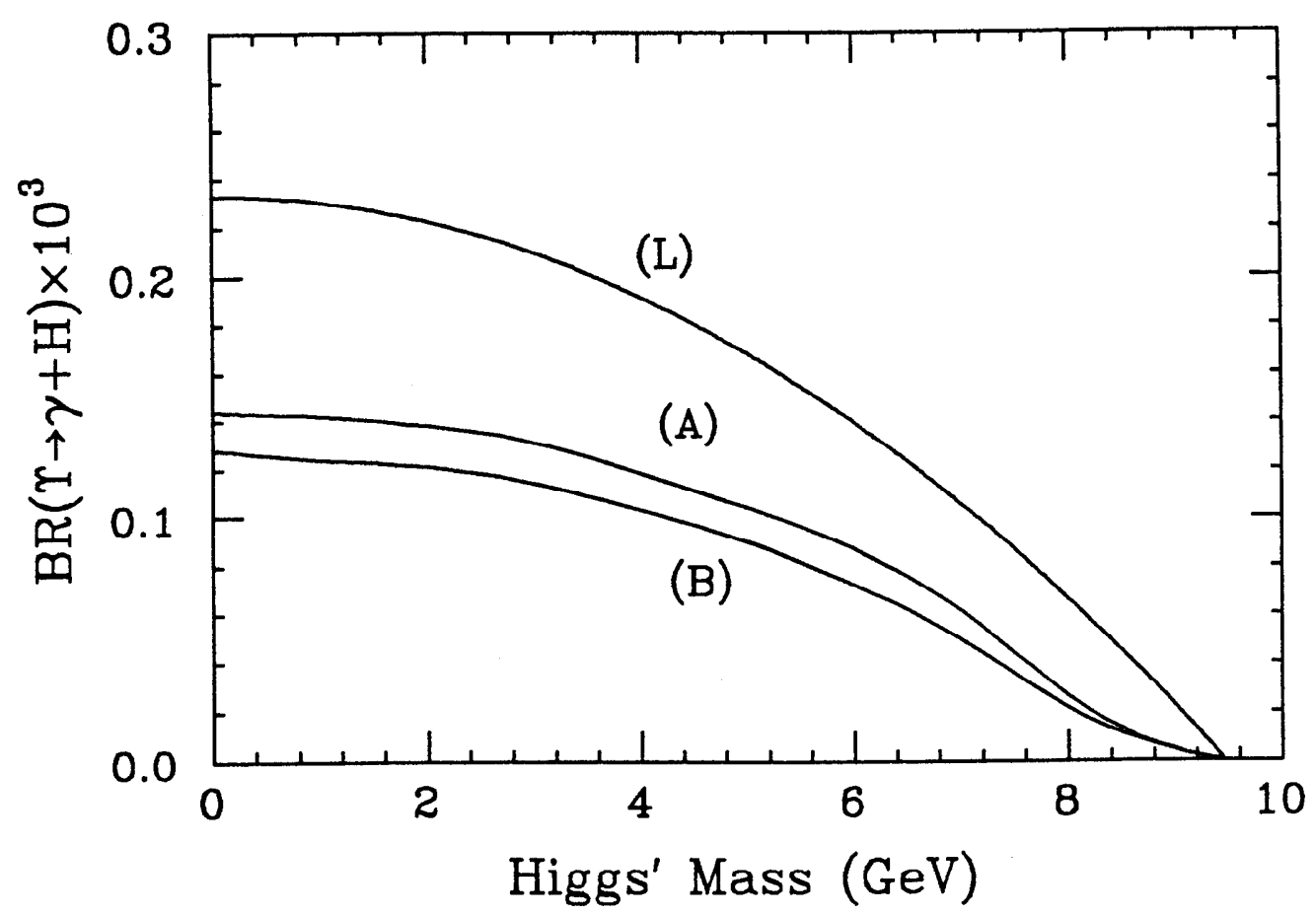

Fig. 1.4 The branching ratio for $\Upsilon \rightarrow \gamma+H$ predicted by the Wilczek mechanism. The leading order (L), and two methods of calculating the first order radiative QCD corrections (A and B) are shown. 


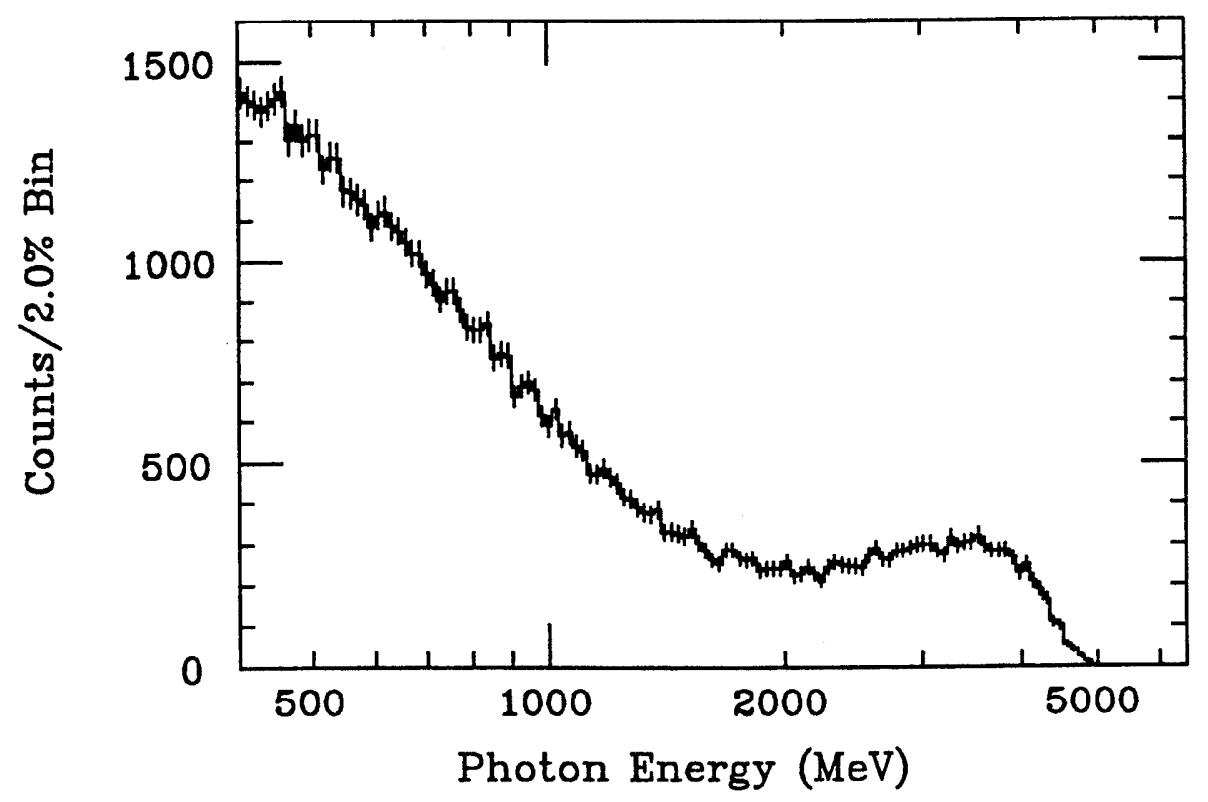

Fig. 1.5 The final $\Upsilon(1 \mathrm{~S})$ inclusive photon spectrum. No obvious narrow structures consistent with the decay $\Upsilon \rightarrow \gamma X$ are indicated.

\subsection{Experimental Results}

Results from this experiment, detailed in Chapter 4, and similar analyses from other experiments are reviewed and compared here. A result of this thesis is the inclusive photon spectrum from the $\Upsilon(1 S)$, shown in Figure 1.5. No narrow resonances are observed in this spectrum so an upper limit for the process $\Upsilon(1 \mathrm{~S}) \rightarrow \gamma X$ is derived. The detection efficiency for this process depends on the decay modes of the state $X$. The most interesting case is where $X$ is a minimal Higgs boson which decays into fermion-antifermion pairs with couplings proportional to the fermion mass. Modeling the detection efficiency consistent with these Higgs decay modes leads to the upper limit shown in Figure 1.6. Figure 1.7 shows the upper limits as a function of recoil mass. The $90 \%$ confidence level upper limit just touches the lowest-order Wilczek calculation for a Higgs' mass in the $5.5 \mathrm{GeV} / \mathrm{c}^{2}$ region. For Higgs' masses below about $4 \mathrm{GeV} / \mathrm{c}^{2}$, the efficiency drop, due to Bhabha rejection criteria, raises the corresponding upper limit. The decay modes open to the Higgs for masses below about $1 \mathrm{GeV} / \mathrm{c}^{2}$ are 


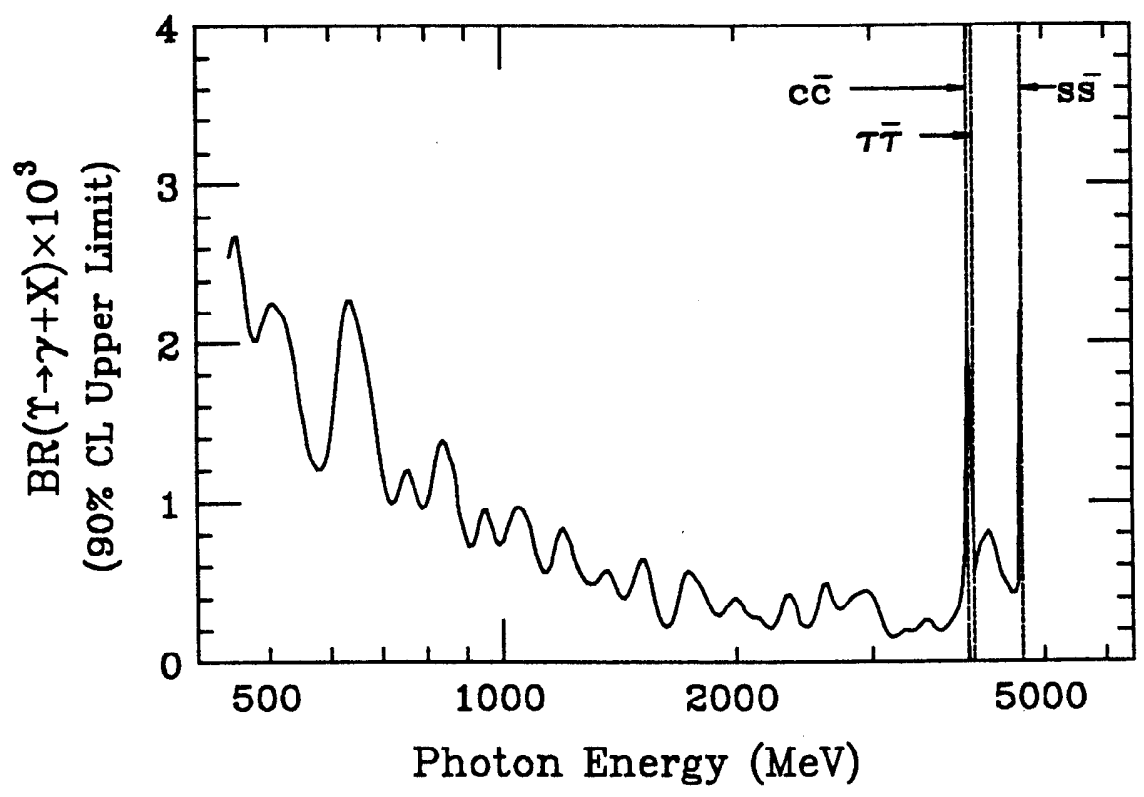

Fig. 1.6 The 90\% confidence level upper limit for the process $\Upsilon(1 \mathrm{~S}) \rightarrow$ $\gamma X$ as a function of photon energy. The assumptions on the decay of $X$ are found in Chapter 4. The vertical dashed lines show the kinematic thresholds for the relevant fermions.

sufficiently different from the decays into $c \bar{c}$ and $s \bar{s}$ that an entirely different analysis would be required to separate such decays from QED processes. For Higgs' masses above $5.5 \mathrm{GeV} / \mathrm{c}^{2}$, the efficiency drop for lower energy photons and higher backgrounds combine to quickly raise the corresponding upper limit. This effect, along with the decrease in theoretical estimates of the branching ratio, make it extremely difficult to rule out a Higgs mass above about $8 \mathrm{GeV} / \mathrm{c}^{2}$.

The other experiments which have reported similar results are the ARGUS collaboration which also uses data taken at the DORIS II storage ring and the CUSB and CLEO experiments which run at the CESR storage ring at Cornell University. Figure 1.8 shows the $90 \%$ confidence level upper limits for $B R(\Upsilon \rightarrow$ $\gamma X)$ from the ARGUS experiment ${ }^{26 !}$ The upper plot is derived by detecting photons directly in a shower counter while the lower plot requires the photons to convert to $e^{+} e^{-}$pairs in the beam pipe which are then detected. The CLEO detector, like ARGUS, is a magnetic detector designed to measure the energy 


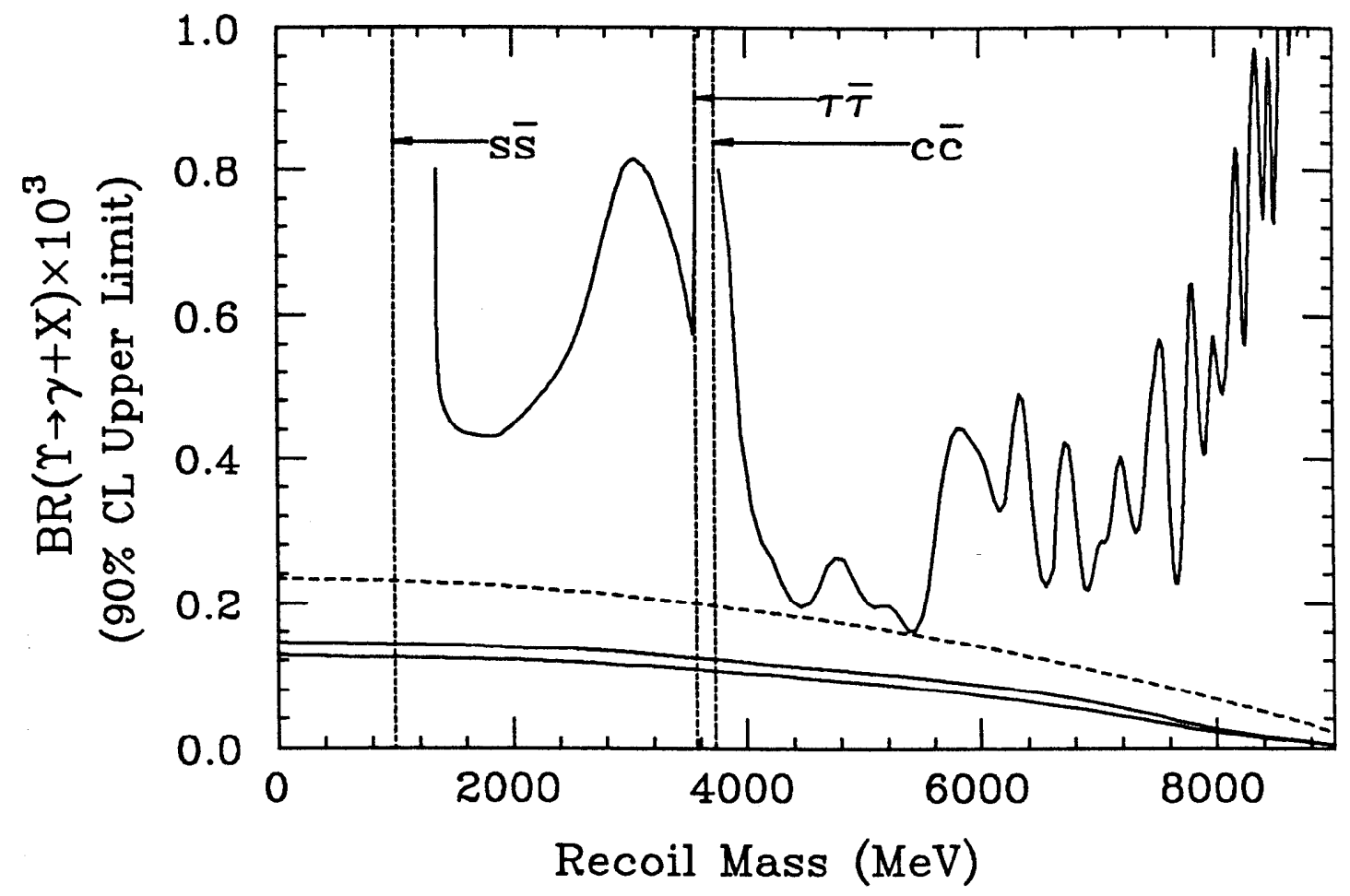

Fig. 1.7 The $90 \%$ confidence level upper limit for the process $\Upsilon(1 \mathrm{~S}) \rightarrow$ $\gamma X$ as a function of recoil mass. The vertical dashed lines show the kinematic thresholds for the relevant fermions. The horizontal dashed line corresponds to the lowest order calculation for the Wilczek mechanism. The two solid horizontal lines indicate the range of the theoretical estimate of the first order radiative corrections to the Wilczek calculation.

and momentum of charged particles. By measuring $e^{+} e^{-}$pairs from converted photons they report the upper limits shown in Figure 1.9.27] These plots should be compared to Figure 1.6. The CUSB detector is similar to the Crystal Ball detector in that it is a non-magnetic calorimeter. Figure 1.10 shows CUSB's upper limit as a function of recoil mass squared!28]

The CUSB result is the most similar to that found in this thesis. Their plot can be compared to Figure 1.7 except that CUSB plots the recoil mass squared. These two results are similar for a Higgs' mass above $4 \mathrm{GeV} / \mathrm{c}^{2}$. The lack of kinematic thresholds in CUSB's plot, however, indicates they must have used a 

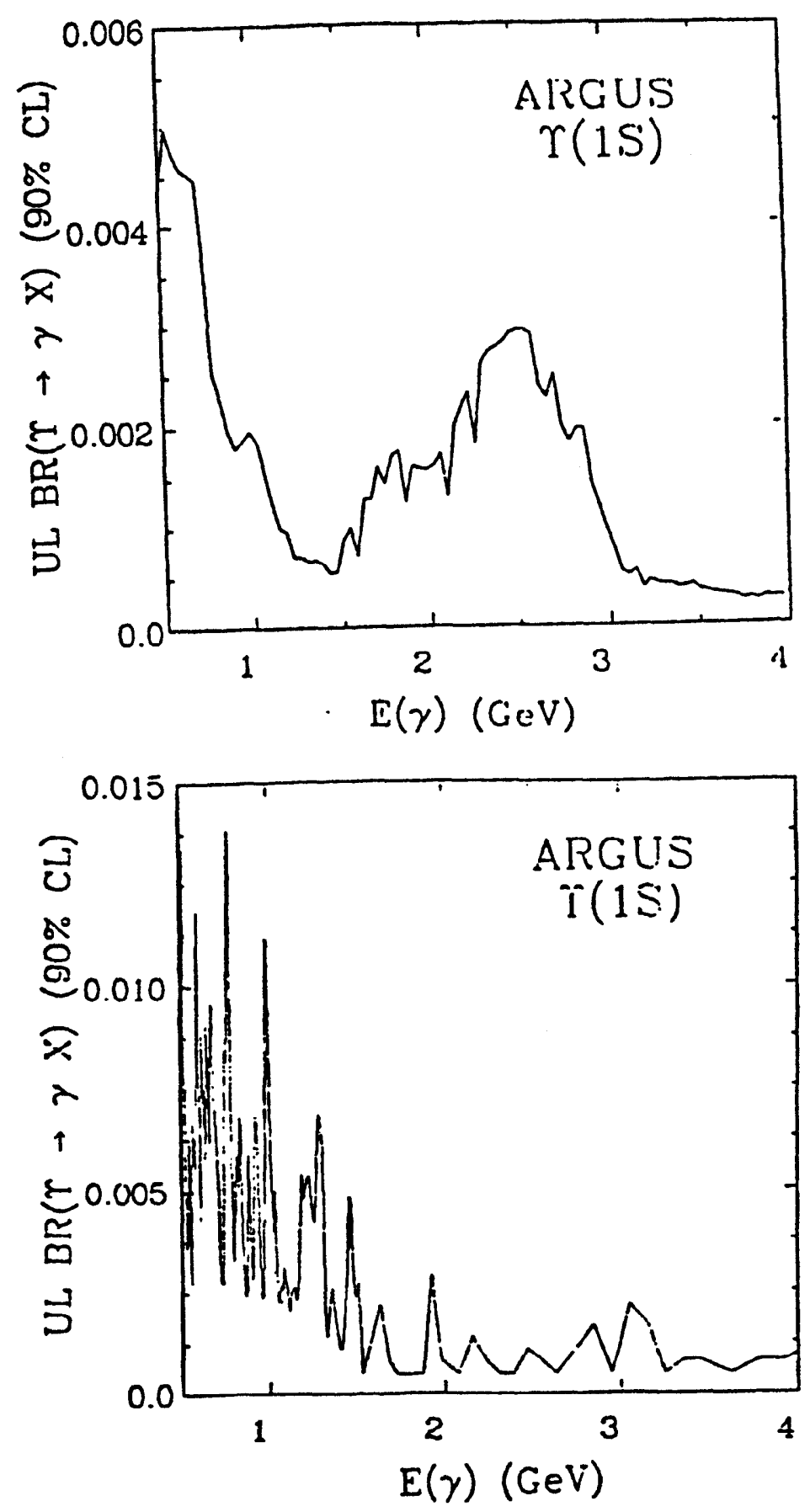

Fig. 1.8 The upper limit for $B R(\Upsilon \rightarrow \gamma X)$ as a function of photon energy reported by the ARGUS collaboration. The top plot is derived from measuring photons directly in their shower counters. The lower plot requires the photons to first convert to $e^{+} e^{-}$pairs in the beam pipe which are then detected. 


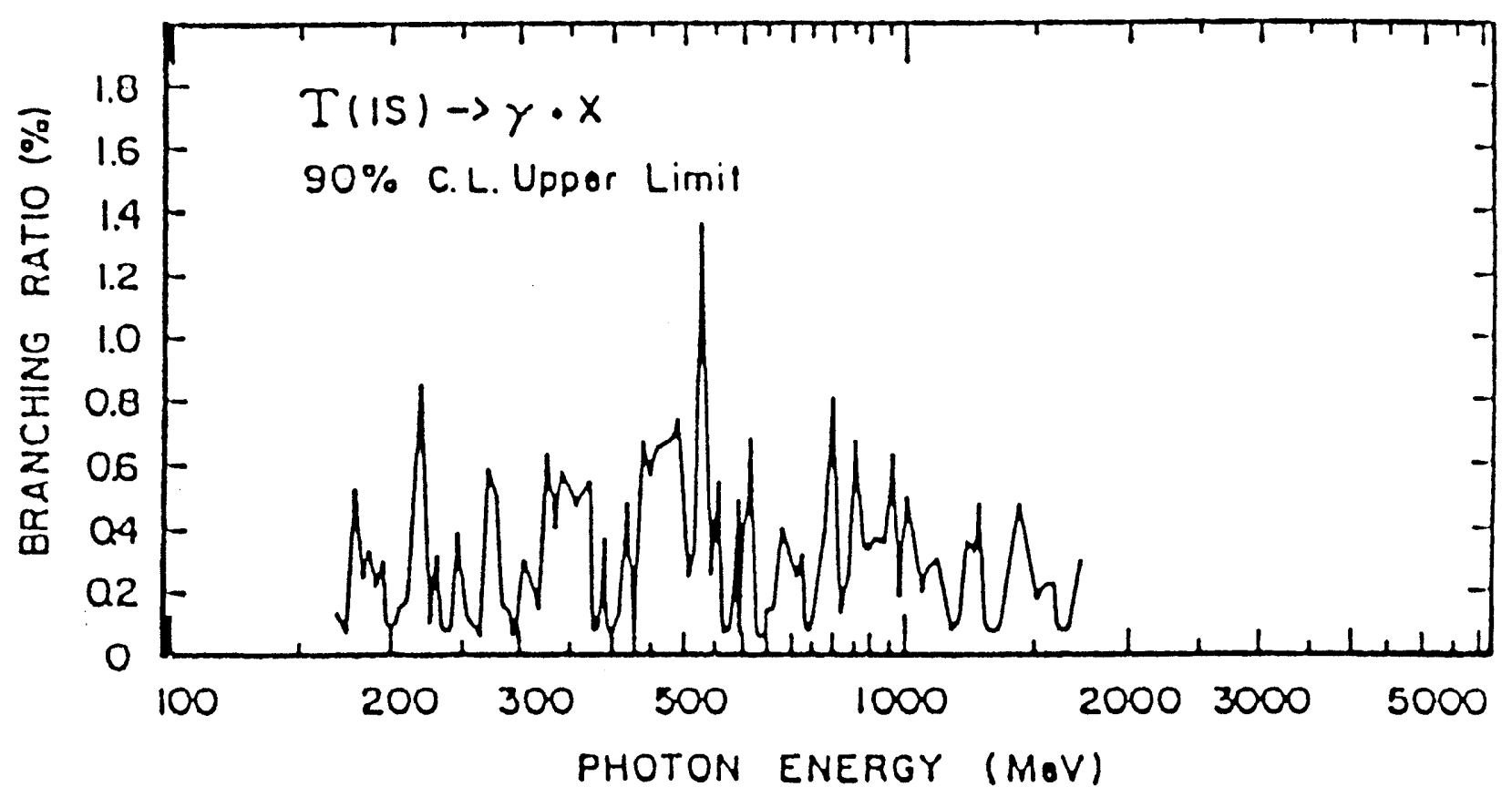

Fig. 1.9 The upper limit for $B R(\Upsilon \rightarrow \gamma X)$ as a function of photon energy reported by the CLEO collaboration. This analysis requires photons to first convert to $e^{+} e^{-}$pairs in the beam pipe which are then detected.

very different model for their efficiency calculation. Thus, for a Higgs' mass below $4 \mathrm{GeV} / \mathrm{c}^{2}$, the comparison between this analysis and CUSB's is not possible.

\subsection{Summary}

The search for the Higgs boson in radiative $\Upsilon$ decays appears the most direct method available for discovering a light Higgs. Unfortunately, no Higgs' masses have been experimentally ruled out by this method. To reach the current theoretical estimates for a Higgs' mass above $8 \mathrm{GeV} / \mathrm{c}^{2}$ would require a sample of about 10 million $\Upsilon(1 \mathrm{~S})$ decays gathered with a detector having a sensitivity 5 times that of the Crystal Ball. This would be an enormous undertaking spanning many years of data-taking. Thus, it will be difficult to substantially improve the result presented here without an enormous increase in the num- 


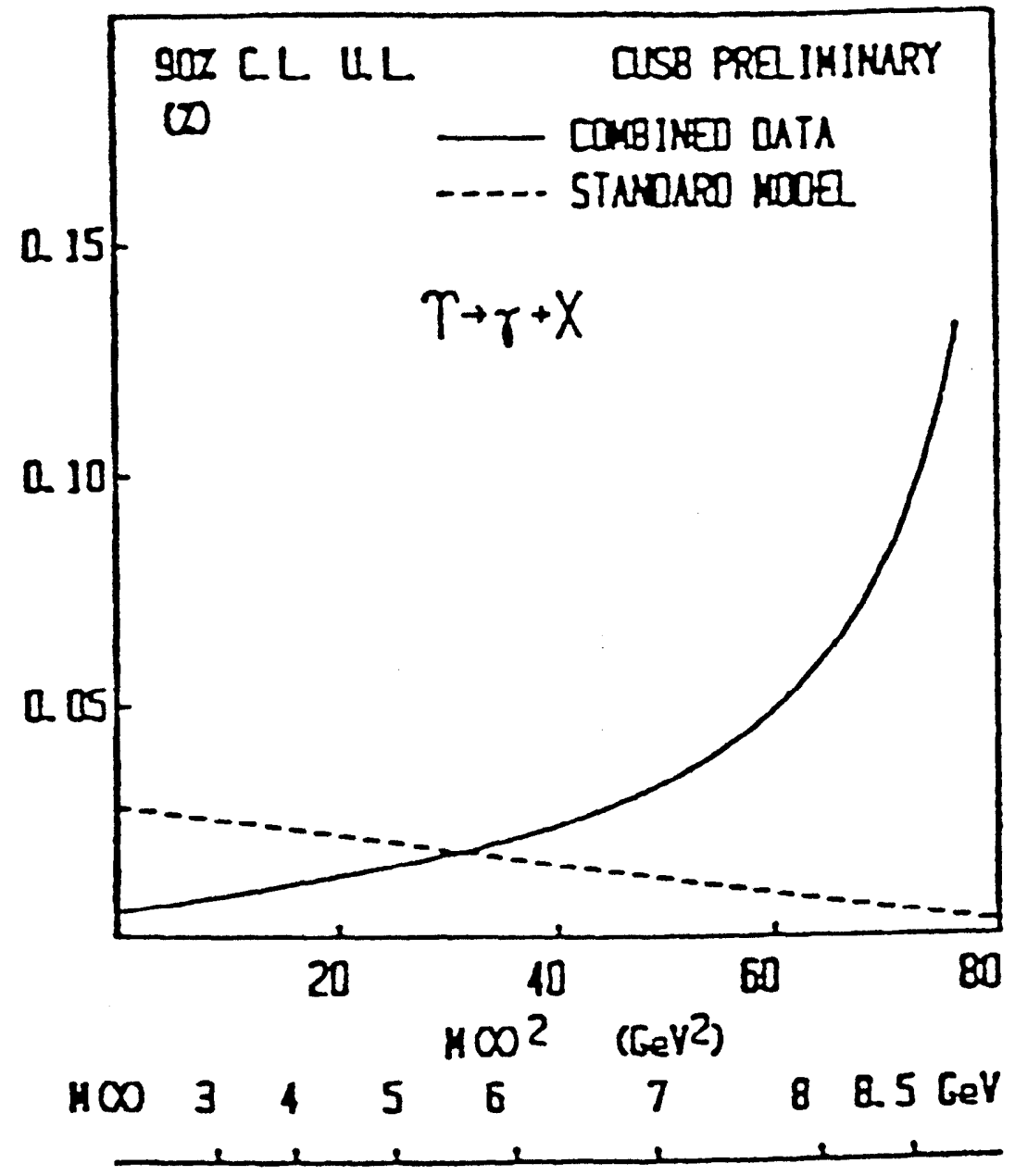

Fig. 1.10 The upper limit for $B R(\Upsilon \rightarrow \gamma X)$ as a function of recoil mass squared reported by the CUSB collaboration. The dashed line shows the lowest order Wilczek calculation.

ber of $\Upsilon$ decays or a significantly better detector than any in existence today. 
1) P. W. Higgs, Phys. Rev. Lett. $\underline{13}$ (1964) 321; Phys. Rev. $\underline{145}$ (1966) 1156; T.W.B. Kibble, Phys. Rev. 155 (1967) 1554.

2) S. Weinberg, Phys. Rev. Lett. 19 (1967) 1264; S. L. Glashow, Nucl. Phys. 22 (1961) 579; A. Salam, Proceedings, 8th Nobel Symposium, edited by N. Svartholm, Stockholm 1968, p367.

3) Numerical constants taken from Particle Data Group, Rev. Mod. Phys. $\underline{56}$ (1984) S31.

4) William J. Marciano and A. Sirlin, Phys. Rev. D29 (1984) 945, Erratumibid D31 (1985) 213.

5) A. Sirlin and W. J. Marciano, Nucl. Phys. B189 (1981) 442.

6) M. Spiro (UA1) and A. Clark (UA2), Proceedings of the 1983 International Symposium on Lepton and Photon Interactions at High Energy, Cornell University, 1983.

7) L. DiLella, Invited talk given at 1985 Int. Symp. on Lepton and Photon Interactions at High Energies, Kyoto, Japan, Aug 19-24, 1985, P279, edited by $\mathrm{M}$. Konuma and $\mathrm{K}$. Takahashi.

8) Paul Langacker, Invited talk given at 1985 Int. Symp. on Lepton and Photon Interactions at High Energies, Kyoto, Japan, Aug 19-24, 1985, and at 4th Symp. on Theoretical Physics, Mt. Sorak, Korea, Aug 1985.

9) William J. Marciano, Phys. Rev. D20 (1979) 274.

10) For a thorough experimental and theoretical review of the Higg's mass, see Ricardo A. Flores and Marc Sher, Ann. Phys. 148 (1983) 95.

11) S. Weinberg, Phys. Rev. Lett. $\underline{36}$ (1976) 294.

12) K.T. Mahanthappa and Marc A. Sher, Phys. Rev. D22 (1980) 1711; Ricardo A. Flores and Marc Sher, Ann. Phys. 148 (1983) 95. 
13) This was calculated by P. H. Frampton, Phys. Rev. Lett. $\underline{37}$ (1976) 1378 and improved by Ricardo A. Flores and Marc Sher, Ann. Phys. $\underline{148}$ (1983) 95.

14) R. S. Willey and H. L. Yu, Phys. Rev. D22 (1982) 3287.

15) John Ellis, Mary K. Gaillard and D.V. Nanopoulos, Nucl. Phys. B106 (1976) 292.

16) Ernest Ma, Phys. Rev. D31 (1985) 1143.

17) Barbara Schrempp and Fridger Schrempp, Phys. Lett. 168B (1986) 259.

18) S. Coleman and E. Weinberg, Phys. Rev. D7 (1973) 1888.

19) L.B. Okun, Closing talk given at 10th Int. Symp. on Lepton and Photon Interactions at High Energy, Bonn, West Germany, Aug 24-28, 1981.

20) J. D. Bjorken, SLAC-198 (1976); J. Finjord, Phys. Scr. 21 (1980) 143.

21) Andreas S. Schwarz, SLAC-PUB-3665, May, 1985.

22) Frank Wilczek, Phys. Rev. Lett. $\underline{39}$ (1977) 1304.

23) M. I. Vysotskii, Phys. Lett. 97B (1980) 159.

24) J. Pantaleone, M. Peskin and S.-H. Tye, SLAC-PUB-3439.

25) John Ellis, K. Enqvist and D. V. Nanopoulos, Phys. Lett. 158B (1985) 417.

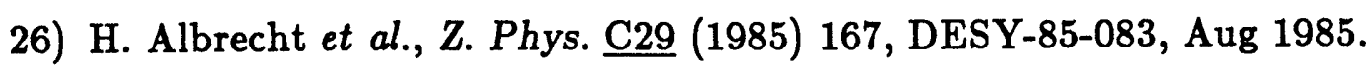

27) D. Besson et al., Phys. Rev. D33 (1986) 300, CLNS-85-701.

28) J. Franzini, Print-85-0764, July 1985, invited talk given at 5th International Conference on Physics in Collision, Autun, France, July 3-5, 1985. 


\section{THE CRYSTAL BALL EXPERIMENT}

\subsection{Introduction}

The Crystal Ball experiment has been officially in progress for eleven years; it is therefore an old-timer as high-energy experiments go. Thus, detailed descriptions covering several aspects of the Crystal Ball have already been written. To avoid unnecessary repetition, detector components described in this chapter and the standard off-line data analysis found in Chapter 3 will be detailed enough to make clear the experimental techniques discussed in this thesis. Reference will be made to the appropriate source(s) for the reader interested in greater detail. Any relevant modifications beyond the referenced descriptions will of course be included here.

\subsection{The DORIS II Storage Ring}

The original DORIS $e^{+} e^{-}$storage ring operated with electrons and positrons circulating in two separate rings, one over the other. These beams crossed at 2 experimental interaction regions to provide $e^{+} e^{-}$collisions up to $7 \mathrm{GeV}$ in the center of mass ${ }^{[1]}$ In order to study the $\Upsilon$ region, the maximum energy was raised to $10.2 \mathrm{GeV}$ in 1978 using the upper of the two rings, converting DORIS to a single bunch, single ring machine. From November 1981 to June 1982, extensive modifications were undertaken to upgrade the machine to DORIS II!2] 


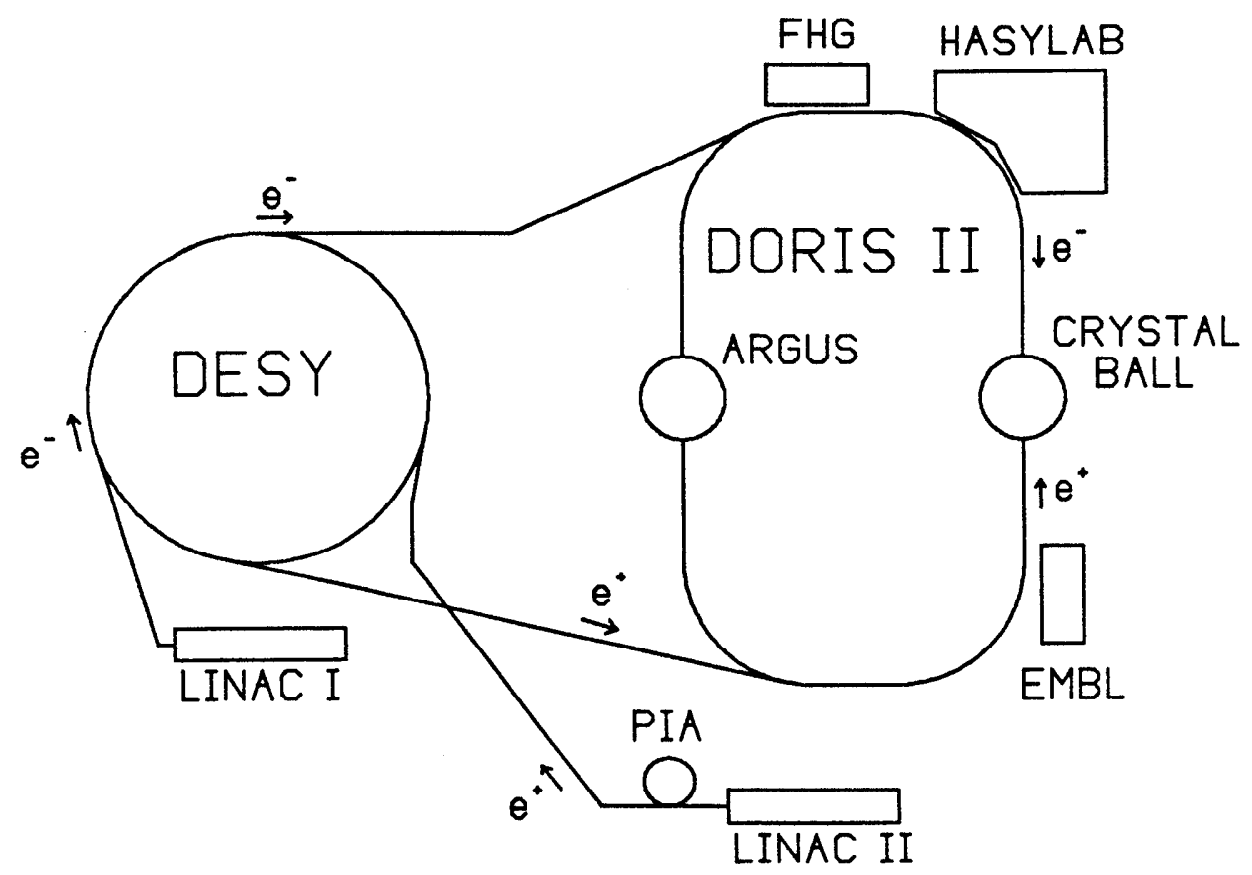

Fig. 2.1 Layout of the DORIS II storage ring. Electrons and positrons from Linac I and II are stored and accelerated at the DESY synchrotron before being transmitted to DESY II.

Bending magnets and RF power supplies were modified or replaced, the lower ring was removed and mini- $\beta$ focusing magnets were installed 1.2 meters from the interaction regions to boost the specific luminosity. The maximum center of mass energy was raised to $11.2 \mathrm{GeV}$ with a decrease in total power consumption.

Figure 2.1 shows the DORIS II injection system. Bunches of $40 \mathrm{MeV}$ electrons from Linac I and $400 \mathrm{MeV}$ positrons, collected from Linac II in the PIA accumulator ring, are transferred to the DESY synchrotron where they are accelerated to the DORIS II beam energy. The electrons and positrons from DESY can then be injected into DORIS II. Because beams do not have to be dumped before injection at DORIS II, the beam currents can be topped off in about 1-2 minutes during normal operation. When DORIS II began operation in 1982, an $8 \mathrm{~cm}$ optic $\left(\beta_{y}^{*}\right)$ was used. This was soon reduced to a $4 \mathrm{~cm}$ optic and resulted in peak luminosities between $(1.5-2.0) \times 10^{31} \mathrm{~cm}^{-2} \mathrm{sec}^{-1}$. 


\section{CRYSTAL BALL}

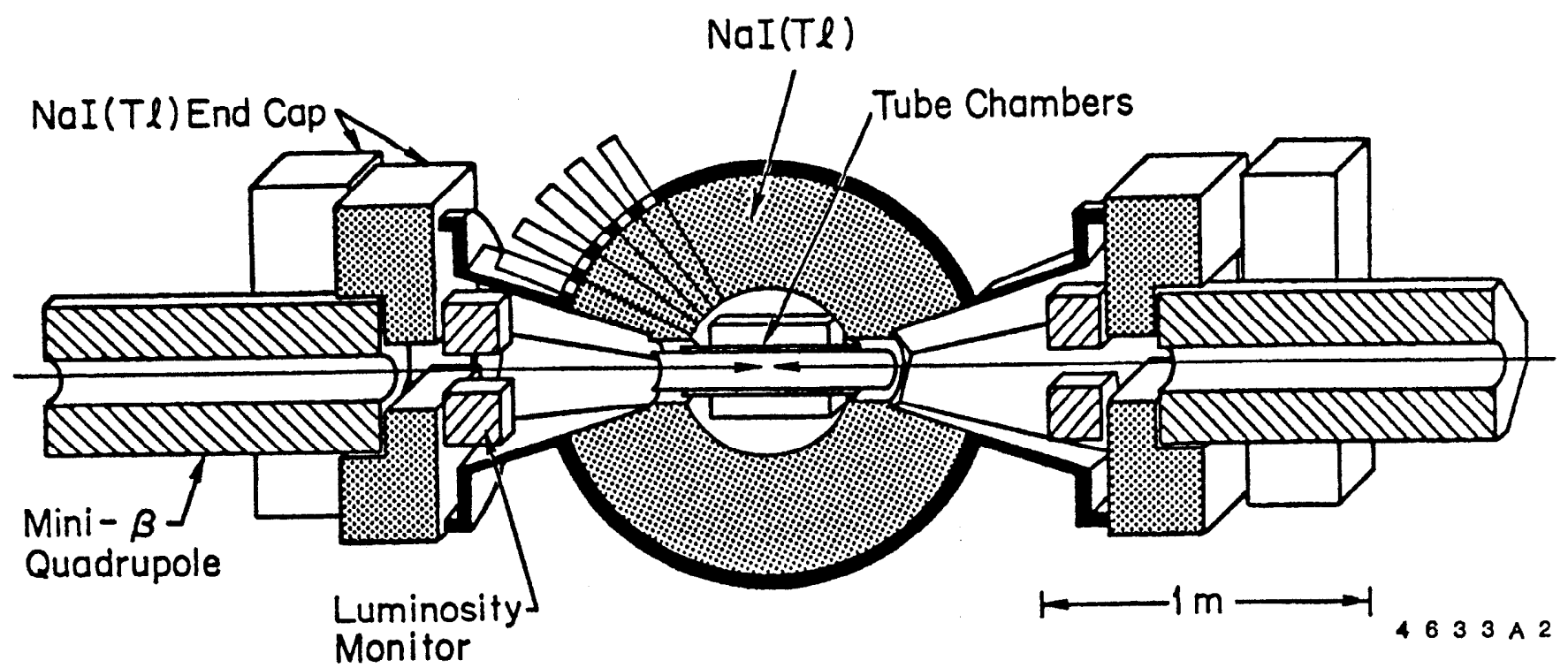

Fig. 2.2 The major components of the Crystal Ball at DORIS II. Timeof-Flight counters, signal processing electronics and data acquisition system are not shown.

Typical $\Upsilon(1 \mathrm{~S})$ running involves injecting to $40 \mathrm{~mA}$ per beam and running down to 20-25 $\mathrm{mA}$ after about $1 \frac{1}{2}$ hours. DORIS II frequently delivers 800 $1000 \mathrm{nb}^{-1}$ per day at the $\Upsilon(1 \mathrm{~S})$, but downtime for start-up and intermittent breakdowns reduce this to about $500 \mathrm{nb}^{-1}$ per day, averaged over a long run.

\subsection{Experimental Overview}

The Crystal Ball detector, shown in Figure 2.2, consists of several major hardware components:
1) The Main Ball
2) Endcap Arrays
3) Central Tracking Chambers
4) Luminosity Counters
5) Time of Flight System 
6) Signal Processing Electronics

7) Online Data Acquisition System

The first 4 components are housed in a $4 \times 4 \times 6$ meter "dry house" which provides a stable temperature and humidity environment for reasons described below. Above and around the sides of the dry house lies the Time of Flight system which is itself surrounded by large concrete blocks for radiation shielding of the experimental hall. The signal processing electronics and the on-line data acquisition system reside in the Crystal Ball control room located about 15 meters above the DORIS ring.

\subsection{The Main Ball}

The primary component of the Crystal Ball detector is the main ball, or Crystal Ball proper. It consists of 672 Thallium doped NaI crystals stacked in a spherical array. $\mathrm{NaI}(\mathrm{Tl})$ is used because of its good energy resolution for electromagnetically showering particles; photons, electrons and positrons. The energy resolution for these particles, determined from studies at SPEAR $?^{[3]}$ was found to be

$$
\frac{\sigma_{E}}{E}=\frac{2.7 \pm 0.2 \%}{\sqrt[4]{E}}
$$

The high segmentation provides an angular resolution of $1-2^{\circ}$ depending on energy; higher energy photons and electrons have the better angular resolution. This segmentation is needed to spatially resolve showering particles from other nearby particles in the $e^{+} e^{-}$interactions studied here.

The geometry of the Crystal Ball is based on the icosahedron as shown in Figure 2.3 $3^{[4]}$ Each of the icosahedron's 20 faces, called "major triangles," are divided into 4 "minor triangles," each of which is itself divided into 9 "modules", or crystals. All crystals are projected onto a 26 inch radius sphere and are truncated 10 inches from the origin. This results in a 10 inch radius spherical cavity 
CRYSTAL BALL

GEOMETRY AND JARGON

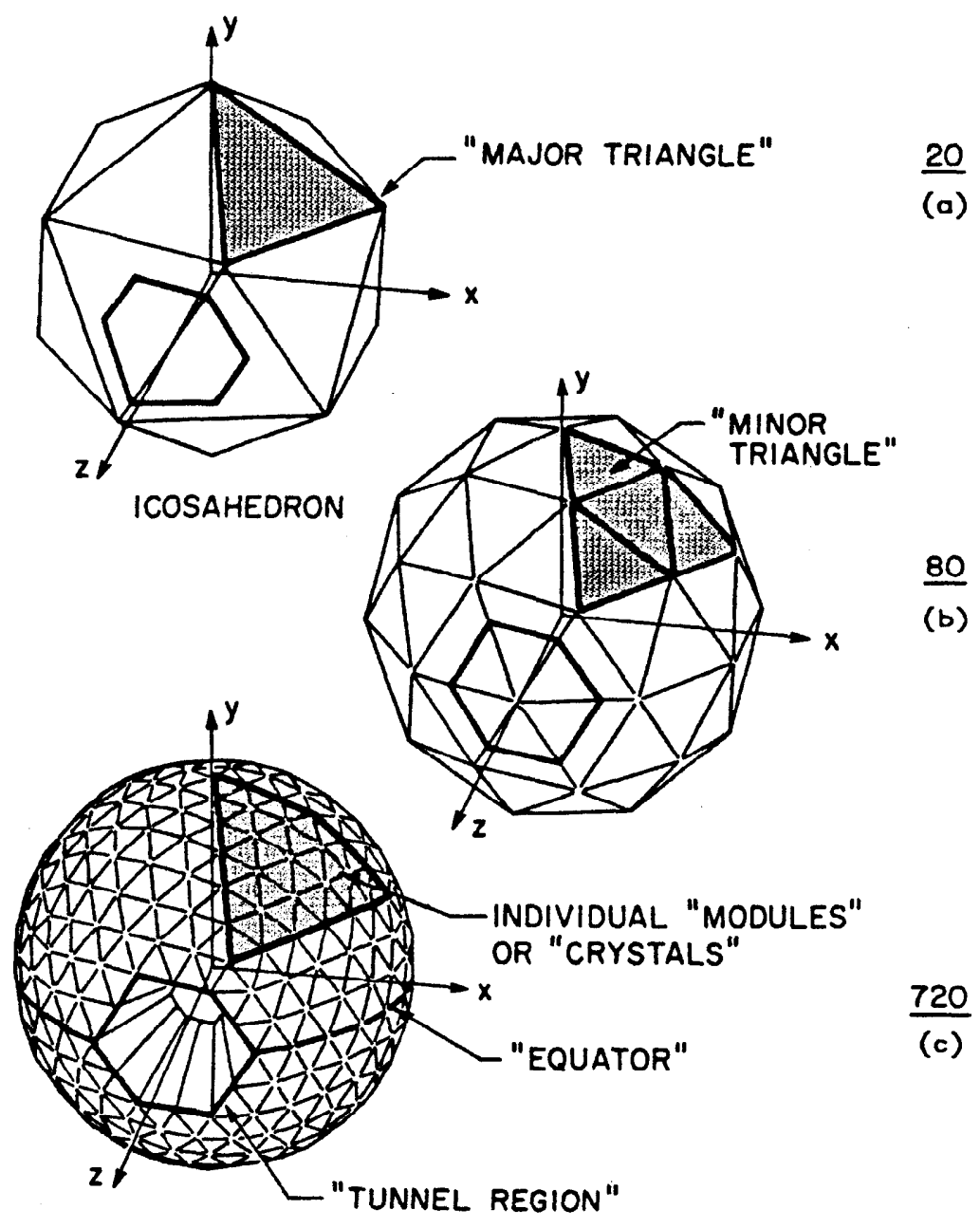

Fig. 2.9 The underlying structure of the Crystal Ball. The top figure shows an icosahedron where each face is termed a major triangle. In the middle figure, each major triangle is divided into 4 minor triangles. The bottom figure shows the geometry of the individual crystals resulting from dividing minor triangles into 9 crystals each. 
inside the ball which provides space for both the beampipe and tracking chambers. Because each crystal is 16 inches long it represents roughly 16 radiation lengths or one nuclear absorption length of $\mathrm{NaI}$.

Of the 720 possible crystals in the geometry described, 48 which would normally lie near the $\pm \mathrm{z}$-axis do not actually exist to allow entry of the beampipe which transports the $e^{+} e^{-}$beams. These holes, or "tunnels", reduce the ball's solid angle coverage to $93 \%$ of $4 \pi$ steradians. The first layer of crystals surrounding the tunnels are called tunnel modules. In the actual ball, crystals are stacked in two hemispheres, split horizontally, so the detector can be opened vertically. This is necessary to allow access to the interaction region and tracking chambers. A photomultiplier tube is mounted on the outside of every crystal to measure the scintillator light produced by particles traversing the NaI. The photomultiplier output is then directed to the signal processing electronics. All signals are digitized and stored for readout by the on-line computer ${ }^{[4]}$

$\mathrm{NaI}$ is susceptible to two environmental dangers which can degrade its optical properties; water and radiation. $\mathrm{NaI}$ is hygroscopic and can be damaged by even a small exposure to atmospheric moisture. For protection against crystal hydration, each hemisphere is hermetically sealed. Additional safety is provided by the dry house surrounding the detector which provides a dehumidified atmosphere and temperature regulation.

Radiation levels at DORIS are much higher than at SPEAR so several techniques were introduced at DORIS and are presently utilized to reduce the crystal's radiation exposure to a tolerable level. The ball is opened remotely during injection of the $e^{+} e^{-}$beams to distance it from the high radiation levels near the beampipe. In addition, lead shielding is inserted remotely between the opened ball and the interaction region. After injection, the lead is removed and the ball closed. These precautions reduce the injection radiation dose by about a factor of 80. Averaged over a long running period, the ball receives about 1 rad per $\mathrm{pb}^{-1}$ of luminosity. When no data is being taken, during machine studies or 
synchrotron radiation running, a more substantial lead shield is stacked around the interaction region to provide additional protection for the opened ball. Up to this point the total accumulated radiation in the ball, mainly in the regions of the tunnel modules, is 850 rads; approximately 300 rads were received at SPEAR and about 550 rads at DORIS. Significant resolution degradation is expected for doses greater than about 1000 rads, however, no significant performance degradation has been observed at the present time.

\subsection{Endcaps}

To further improve the Crystal Ball's solid angle coverage, NaI endcap arrays are placed away from the main ball along the \pm z-axis. Both NaI endcap arrays use 20 crystals each to provide about 10 radiation lengths over their portion of the solid angle, making the total solid angle coverage about $98 \%$ of $4 \pi$ steradians ${ }^{[5]}$ Many exclusive analyses use energy deposition in the endcaps as an event veto but the inclusive analysis presented here uses them only when calculating the detector's total energy deposition.

\subsection{Tracking Chambers}

The Crystal Ball tracking chambers at DESY consist of proportional tube chambers placed cylindrically in several layers around the interaction region, as shown if Figure 2.4. Each chamber consists of a thin aluminum tube with a $50 \mu$ cathode sense wire stretched across the tube's long axis. Pulse heights were first obtained by using "magic gas" (75\% Argon, 20\% Isobutane, 4\% Methynal and $0.25 \%$ Freon) and later with an Argon- $\mathrm{CO}_{2}$-methane mixture (79\% Argon, $20 \% \mathrm{CO}_{2}$ and $1 \%$ Methane). Pulses from each tube above a software threshold are termed hits. The $\rho$ and $\varphi$ coordinates for each hit are determined by the tube while the $\mathrm{z}$ information is obtained by charge-division readout. For charged particles traversing the chambers, the average $\varphi$ resolution is $1-2 \%$ of $2 \pi$ and the $\mathrm{z}$ resolution is about $1-2 \%$ of the tube length[e] 


\section{2-1983 CONFIGURATION}

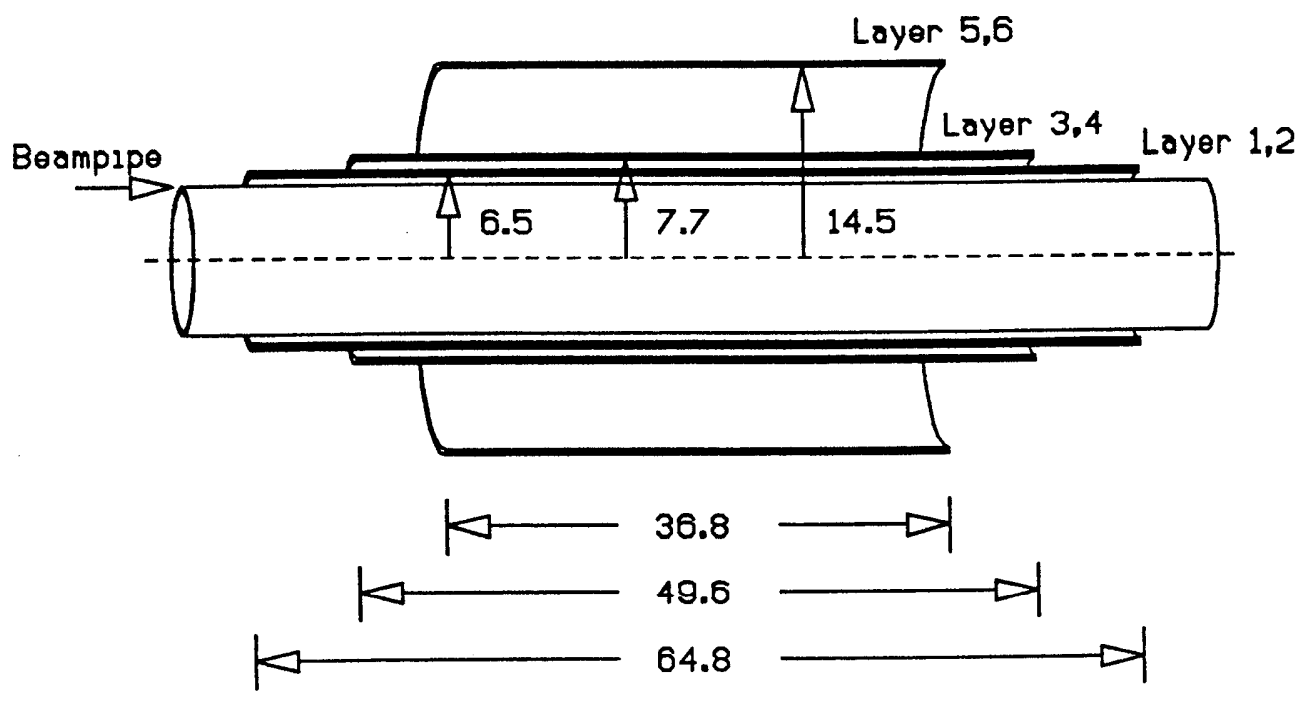

\section{4-1986 CONFIGURATION}

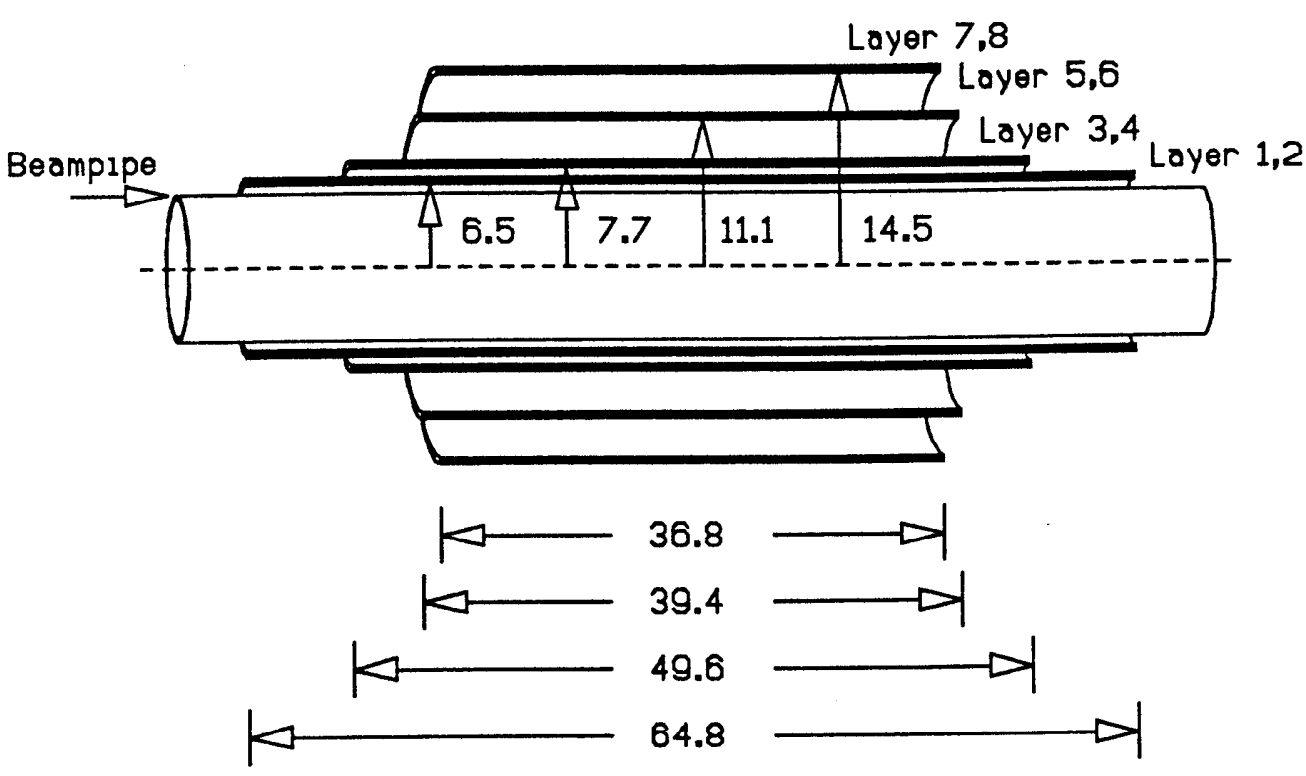

Fig. 2.4 The Crystal Ball tube chambers. The upper figure shows the chamber configuration used from June 1982 until April 1984. The lower figure shows the chambers as they have been from April 1984 to the present. Dimensions are given in centimeters. 
The chambers initially installed at DORIS II ran with magic gas and consisted of three double layers of 3 mil wall aluminum tubes. The inner 4 layers had 80 tubes each while the outer two layers had 160 tubes each. The solid angle coverage from the inner to the outer double layers was 98,96 and $78 \%$ of $4 \pi$ steradians. The magic gas and high radiation environment combined to produce organic compounds on the tube's sense wires. This caused the efficiency to steadily declined until June 1983 when the inner two double layers were replaced. The gas mixture for these chambers was switched to the Argon- $\mathrm{CO}_{2}-\mathrm{Methane}$ mixture $^{|7|}$ and the tube wall thickness was increased to $7 \mathrm{mil}$ to increase mechanical stability. The tube diameter for the inner two layers was also enlarged so each layer contained 64 tubes. In April 1984, the outer double layer was replaced by two double layers, with 112 and 148 tubes per layer respectively, making eight layers in all. This was done to replace the deteriorating layers still using the magic gas mixture and to increase the chamber's tracking ability. The Argon- $\mathrm{CO}_{2}$-methane mixture was then used for all tubes. No degradation of the chamber performance has been seen since changing to the Argon-CO $\mathrm{O}_{2}-$ Methane gas mixture. The solid angle coverage for this configuration was then $98,96,87$ and $78 \%$ of $4 \pi$ steradians respectively. Table 2.1 lists the dimensions and radiation lengths at normal incidence for each double layer of the tracking chambers.

\subsection{Luminosity Counters}

The luminosity monitor is used to cross-check the luminosity measurement made with large-angle Bhabhas in the main ball[ ${ }^{[8]}$ This is accomplished by detecting small angle Bhabhas in the luminosity counters and scaling with the known QED cross-section integrated over the counter's acceptance. The luminosity counters also check the machine performance over short time periods by ensuring the luminosity is delivered properly. Data from the luminosity monitor were not directly used in this analysis. 


\begin{tabular}{|cccccc|}
\hline \multicolumn{6}{|c}{ TRACKING CHAMBER DIMENSIONS AND RADIATION LENGTHS } \\
Youble & $\begin{array}{c}\text { Length } \\
\text { Year }\end{array}$ & Layer & Radius & & $\begin{array}{c}\text { Radiation } \\
\text { (cm) }\end{array}$ \\
\hline \multirow{2}{*}{$1982-83$} & 1 & 64.8 & 6.5 & 0.980 & $4.7 \%$ \\
& 2 & 49.6 & 7.7 & 0.955 & (total) \\
& 3 & 36.8 & 14.5 & 0.785 & \\
\hline \multirow{2}{*}{$1984-86$} & 1 & 64.8 & 6.5 & 0.980 & $7.5 \%$ \\
& 2 & 49.6 & 7.7 & 0.955 & (total) \\
& 3 & 39.4 & 11.1 & 0.871 & \\
& 4 & 36.8 & 14.5 & 0.785 & \\
\hline
\end{tabular}

Table 2.1 A list of dimensions and Radiation Lengths for the Crystal Ball tracking chambers. The angle $\theta_{\min }$ measures the minimum angle from the beam pipe which intersects the chamber. The radiation lengths are calculated for normal incidence and do not include the $1.7 \%$ contribution from the beam pipe.

\subsection{Time of Flight System}

Surrounding the upper portion of the dry house are 94 plastic scintillation counters used to measure the time-of-flight for particles leaving the detector and for identifying cosmic muons. About $50 \%$ of $4 \pi$ steradians are covered by these counters. Because the $\theta$ distribution for cosmic radiation is peaked in the vertical direction, the counters have an effective solid angle coverage of about $80 \%$ for such backgrounds. These counters are primarily used to reject cosmic backgrounds for low multiplicity final states and are not used in this analysis $[?]$

\subsection{On-line Data Acquisition System}

The on-line data acquisition system is responsible for reading data from the detector electronics for each event, providing important statistics about the de- 


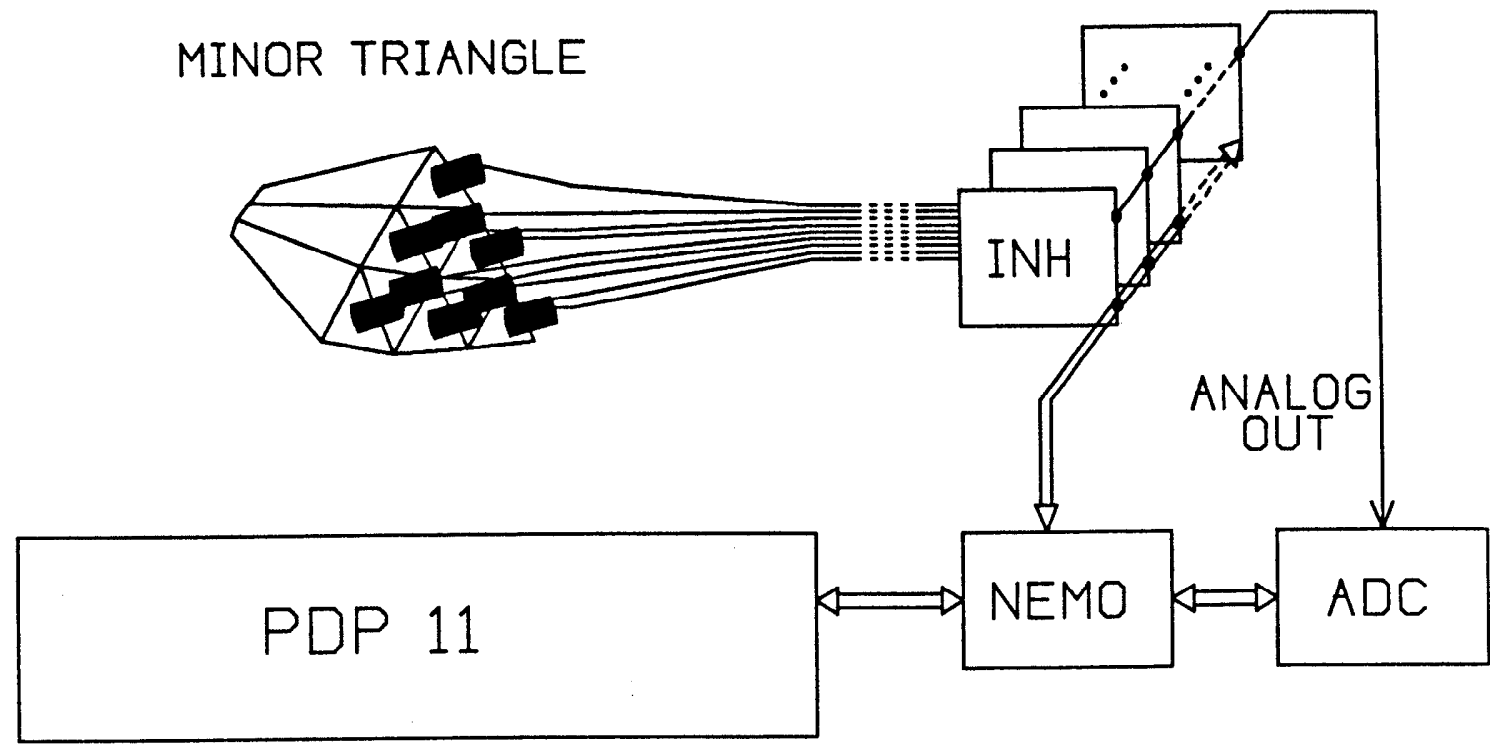

Fig. 2.5 The Crystal Ball on-line data acquisition system. Information from the Crystal Ball detector is read by the PDP-11 on-line computer. The data are collected and sent to the DESY mainframe computer (not shown).

tector performance for physicists on shift, and transferring the data to the DESY mainframe computer, and to magnetic tape if necessary. Part of this system is shown schematically in Figure 2.5. This process must be initiated by a "trigger" in at least one of two independent and somewhat redundant trigger systems. The various classes of physics events desired are chosen and the set of hardware requirements needed to select these events are determined. These trigger systems contain logic circuits which ensure the various hardware requirements are met before "triggering" the on-line computer to read in the data relevant for that event!10] The only trigger requirement needed to select events for this analysis is the "total energy" trigger. This trigger is implemented in hardware by summing the crystal pulse heights from the main ball, minus the tunnel modules, and asserting the trigger if their sum is greater than the trigger threshold. In terms of energy, this trigger begins turning on around $1800 \mathrm{MeV}$ and is $100 \%$ efficient at around $2000 \mathrm{MeV}$ in the main ball. For the hadronic events used in this analysis, 
the trigger is approximately $100 \%$ efficient.

A special trigger needed to simulate machine-related backgrounds is the "DBM" (DORIS Bunch-Marker) trigger. This trigger is asserted every $10^{7}$ beam crossings regardless of other hardware conditions. These DBM events are used to more realistically model beam-related backgrounds in Monte Carlo simulations.

Once an event has triggered the experiment, the PDP-11/T55 on-line computer directs the reading of the ADC's, TDC's, scalars and other information relevant to the event: These data are compressed and written to a large buffer on disk or tape, if necessary, as a backup. The disk buffer is periodically transferred via a data link to DESY's central computer where the data are collected and copied to tape. These tapes are packaged and sent to SLAC for the detailed off-line analysis. Randomly chosen events are analyzed on-line by the Crystal Ball "pipeline." The pipeline generates statistics from these events so those on shift can identify and correct machine, detector and hardware problems as they occur. More subtle problems can be found with the BOL (Bicycle On-Line) job. This job runs on DESY's mainframe computer and checks data sent on the data link from the PDP-11. BOL does a more sophisticated analysis of the data's quality so problems not seen on-line at data taking time can still be found with less than a 24-hour turnaround time.

In order to run user analysis jobs as soon a possible after data taking, special "fast" samples, usually hadrons and special low multiplicity events, are selected and processed when the data arrives on DESY's mainframe computer. This allows users to look for any problems in the data, debug analysis software and get preliminary physics results.

\footnotetext{
* Greater detail on the crystal electronics and signal processing can be found in Appendix
} $A$ in relation to a hardware failure affecting part of the data used in this analysis. 


\begin{tabular}{|cccc|}
\hline Channel & MeV/ADC Count & Energy Range & Pedestal \\
\hline Low & $\sim 0.04$ & $0-325 \mathrm{MeV}$ & $\sim 45$ counts \\
High & $\sim 0.80$ & $0-6500 \mathrm{MeV}$ & $\sim 45$ counts \\
\hline
\end{tabular}

Table 2.2 The nominal values of constants used in the crystal energy calculation. The actual values for each crystal differ somewhat from these nominal numbers.

\subsection{Off-line Crystal Calibration}

The NaI crystal calibration is important for both setting the energy scale and optimizing the resolution of the Crystal Ball. The calibration procedure at DORIS is essentially the same as that at SPEAR!4] This calibration process depends heavily on how the photomultiplier tube (PMT) outputs are converted to energy. To increase the electronic's dynamic range, the PMT pulse height is split into two channels termed the "low channel" and the "high channel". The gain of the low channel is about a factor of 20 higher than the high channel. To determine the high and low channel gains, the ratio of low-channel gain to high-channel gain is found first by comparing ADC values which are common to both channels. The high channel gain, and thus the low channel gain by the relation

$$
\text { low-channel gain }=\text { high-channel gain } \times \text { gain ratio, }
$$

is derived from Bhabha samples where each electron's energy is constrained to the beam energy. One important change made for DORIS data taking was the lowering of the phototube gains by about a factor of two. This was done so that the maximum expected energy in a single crystal at DORIS, taken to be about $6500 \mathrm{MeV}$, would be in range of the on-line electronics. Table 2.2 summarizes the nominal gains and pedestals for the low and high channels along with the corresponding energy ranges. 


\subsection{Crystal Ball Jargon}

Some simple jargon, based on the Crystal Ball geometry, has evolved to describe a class of commonly used cut parameters. Figure 2.6 shows a projection of the Crystal Ball with the deposited energy in each crystal indicated in MeV. The quantity $\mathrm{E} 1$ is defined as the energy in the center crystal with the highest energy deposition; this crystal is always a "bump" as described in Chapter 3. E2 is the sum of E1 and the highest crystal energy in the 12 neighboring crystals (in some cases the central crystal lies in the corner of a major triangle, resulting in only 11 neighbors). E4 is the sum of E1 and the energy in three nearest neighboring crystals. The sum of the energies in the nearest 12(11) neighboring crystals with $\mathrm{E} 1$ is called E13. E13, with small corrections described later, is defined as the energy of an electromagnetically showering particle in the Crystal Ball. 


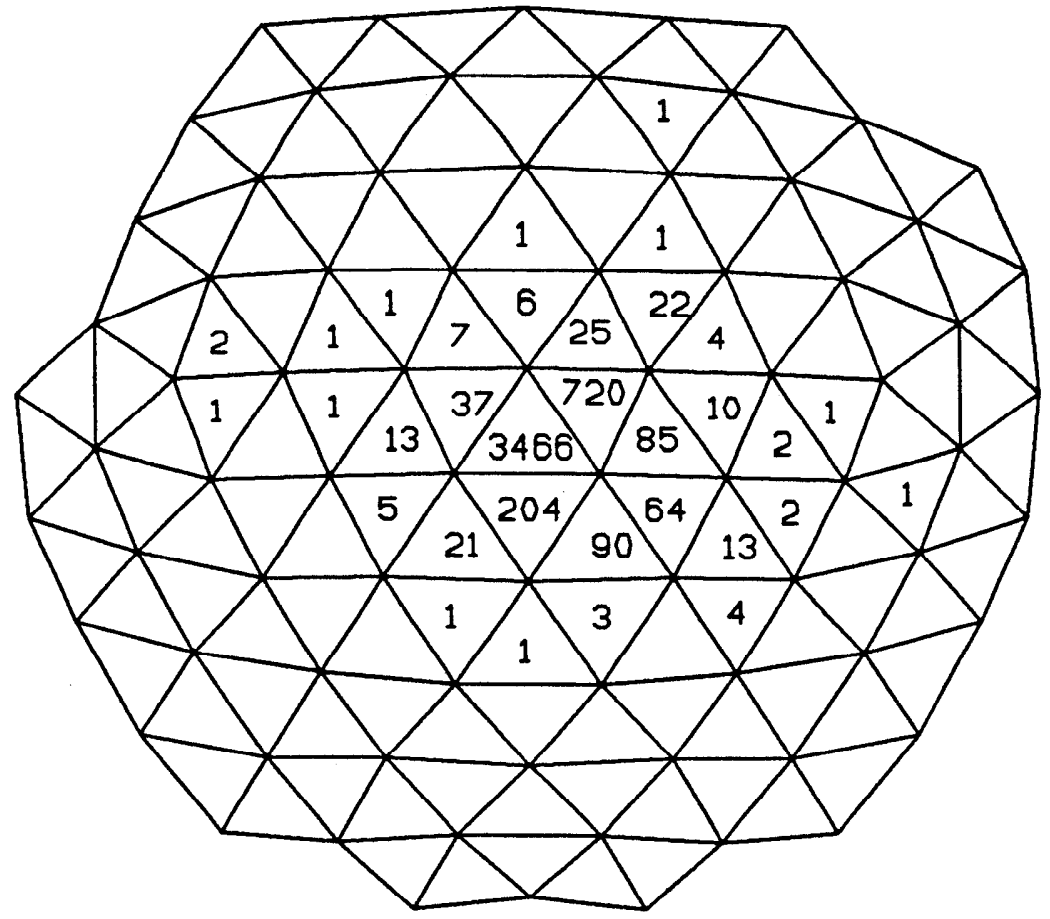

E1

(3466 MeV)

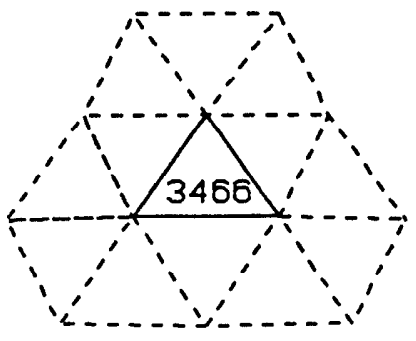

E4

$(4427 \mathrm{MeV})$

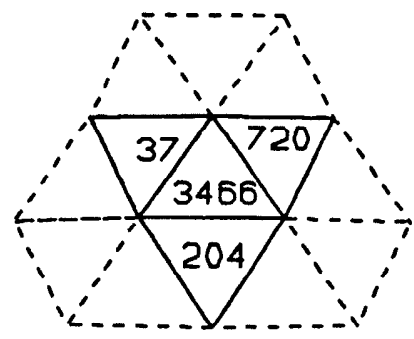

E13

(4743 MeV)

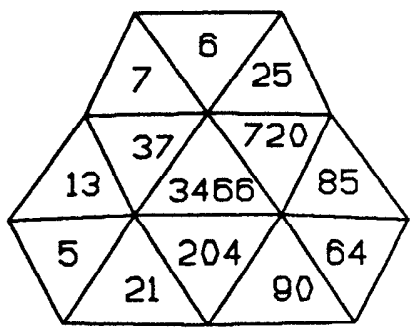

Fig. 2.6 A close-up projection of the Crystal Ball for a typical EM shower. The numbers represent $\mathrm{MeV}$ deposited in each crystal. The upper figure shows a typical electromagnetic shower. The lower figures show the geometrical definitions of E1, E4 and E13. 


\section{References}

1) K. Wille, DESY report number DESY M-81-047 August, 1981.

2) H. Nesemann, K. Wille, DESY report number DESY M-83-026 March, 1983 and DESY M-83-026 August, 1983.

3) J. Gaiser, Ph.D. Thesis, "Charmonium Spectroscopy from Radiative Decays of the $J / \psi$ and $\psi^{\prime}$," Stanford University, 1982, SLAC Report 255 (unpublished).

4) M. Oreglia, Ph.D. Thesis, "A Study of the Reaction $\psi^{\prime} \rightarrow \gamma \gamma \psi$," Stanford University, 1980, SLAC Report 236 (unpublished).

5) A detailed description of the Crystal Ball endcaps can be found in J. S. Leffler, Ph.D. Thesis, "A Search for Primarily Non-Interacting Decay Modes of the Upsilon" Stanford University, 1986, SLAC Report 293 (unpublished).

6) R. Horisberger, Ph.D. Thesis, "Search for Charmed F Mesons in $e^{+} e^{-}$ Collisions with the Crystal Ball," Stanford University, 1984. SLACReport-266 (unpublished).

7) The Argon- $\mathrm{CO}_{2}$-methane mixture was chosen because SLAC's HRS collaboration found it to be satisfactory in high radiation environments.

8) For details on the luminosity monitor see D. Andreasyan, J. Irion and A. Weinstein, Crystal Ball note 317, 1982; J. Irion, Crystal Ball note 322, 1982. Details on the large angle luminosity measurement from Bhabhas can be found in G. Folger, J. Irion and B. Lockman Crystal Ball note 600, 1984.

9) D. Prindle, Ph.D. thesis, "Measurement of the Resonance Parameters of the $\Upsilon$ and $\Upsilon^{\prime}$ Mesons," Carnegie-Mellon Univsity, 1985 (unpublished).

10) G. Godfrey, Crystal Ball note 131, 1978; J. S. Leffler, Ph.D. Thesis, "A Search for Primarily Non-Interacting Decay Modes of the Upsilon" Stanford University, 1986, SLAC Report 293 (unpublished). 


\section{DATA PROCESSING}

\subsection{Off-line Analysis}

After ADC and TDC information and other data are written to tape, the job of reconstructing the event falls on the off-line analysis! ${ }^{[1,2]}$ The Crystal Ball offline analysis software consists of six major production steps shown in Table 3.1. In general, each step extracts progressively more sophisticated information from the raw data and the results of previous steps. The major goal is to identify particles and correctly measure their energy, direction and charge. Because the Crystal Ball is a non-magnetic calorimeter, charged hadron momenta cannot be measured. Thus, particle identification is accomplished by observing the shower distribution in several neighboring crystals.

The first step in the analysis, called ENERGY, determines the energy deposited in each of the $672 \mathrm{NaI}$ crystals. The analog signals from the crystal phototubes are processed and held on capacitors for each electronic channel. These channels are split into a high and low channel, as explained in Chapter 2, and sequentially digitized by a 13-bit ADC (8191 counts). The high channel is used to calculate the crystal energy if either the high channel ADC counts are above 350 or the low channel counts are above 7000 (both of these values nominally correspond to $280 \mathrm{MeV}$ ), otherwise the low channel is used. The energy is then calculated by multiplying the pedestal-subtracted ADC counts by the gain 


\begin{tabular}{|l|c|}
\hline \multicolumn{2}{|c|}{ OFFLINE PRODUCTION STEPS } \\
\hline 1) ENERGY & Convert crystal ADC values to energy in MeV \\
\hline 2) CONREG & Find connected regions \\
\hline 3) BUMPS & Find bumps \\
\hline (EOTAP) & Software trigger and hadron selectors \\
\hline 4) CHGTRK & Charged particle tracking and tagging \\
\hline 5) ESORT & Calculate track energies and directions \\
\hline 6) TOFANL & Time of Flight hits and timing \\
\hline
\end{tabular}

Table 9.1 The major off-line production steps.

for that channel.

Once the crystal energies are known, locating groups of neighboring crystals with deposited energy gives information on particle directions and energies. All crystals with at least $10 \mathrm{MeV}$ belong to exactly one energy cluster called a "connected region." Two crystals with at least $10 \mathrm{MeV}$ each, and which share an edge or corner, belong to the same connected region. Thus, connected regions are analogous to $10 \mathrm{MeV}$ contour lines and help separate nearby energy deposition and suppress low energy noise. The task of locating connected regions in an event is performed by the routine CONREG.

The BUMPS routine finds local maxima in a connected region. The crystal with the greatest energy in a connected region is called a "bump" and is associated with a particle as shown in Figure 3.1. Each bump is placed as an entry in the "track bank," a Fortran common block used to store particle information. The term "track" refers to any entry in the track bank. To determine whether more than one particle may have created the given energy deposition pattern, an empirically determined algorithm ${ }^{[9]}$ decides which crystals have energy consistent with the bump(s) already found in that connected region. If a crystal 


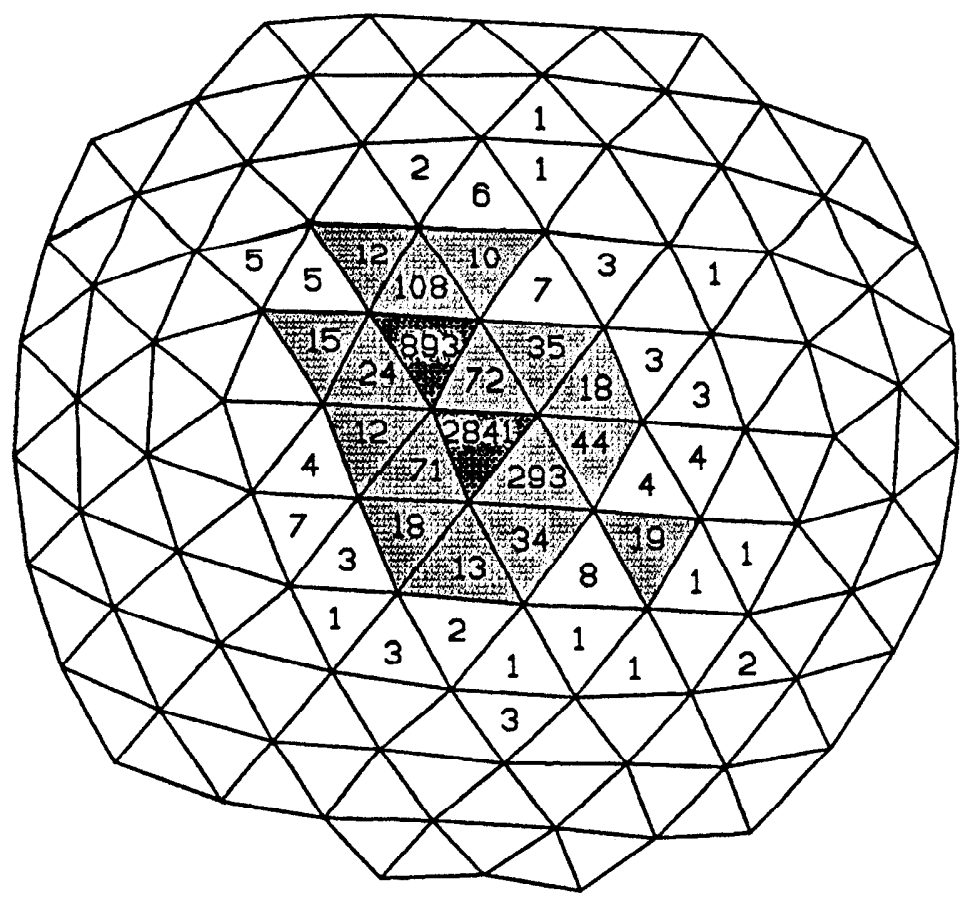

Fig. 3.1 Definition of Connected Regions and Bumps. The numbers indicate energy deposited in each crystal in $\mathrm{MeV}$. Light grey regions show the crystals in the connected region. The dark grey crystals show bumps.

has more energy than can be accounted for from the bump(s) already found, it too is labeled a bump. This process is repeated until all crystals are accounted for. This "bump discriminator" was designed so that shower fluctuations rarely produce extra bumps while the most closely lying particles remain separable.

The tube chamber pulse height information is examined next by CHGTRK in a two-step process. In the first step, charged particle candidates are identified by finding at least three colinear chamber hits. The best candidates based on straight-line fits which pass near the interaction vertex are called "IR tracks." Chamber hits used to find IR tracks are flagged and are not used in subsequent analysis. A fit to all IR tracks is then preformed to determine the event's Zvertex. In the second step, IR tracks and the remaining hits are correlated with 
crystal information, specifically bumps. Bumps correlated with an.IR track are considered charged. IR tracks not associated with a bump are added to the track bank as zero energy tracks. Bumps not correlated with an IR track are compared with the remaining hits. If a correlation is found, the bump is considered charged and termed a "tagged" track, otherwise the bump is neutral.

Another routine used to identify charged particles, called CONTAG, is also used in this analysis. CONTAG calculates the probability that a bump is charged by first calculating the probability that a given chamber hit is correlated with the bump direction, for all hits. The probability the bump is charged is then given by

$$
P_{\text {charged }}=1-\prod_{i=1}^{\# \text { hits }}\left(1-P_{i}\right)
$$

where $P_{i}$ is the probability that the $i^{\text {th }}$ chamber hit is associated with the bump direction. The probability $P_{i}$ is calculated from the measurement errors associated with the bump and hit directions. Before the inner two double layers were replaced in June 1983, CONTAG's tagging efficiency was much better than the production tagging described above. This will be elaborated in greater detail in Chapter 4.

The next step in the off-line analysis, ESORT, determines the energies and directions of all tracks. The track energy is calculated in two ways. The first energy estimation, called ESORT, associates all deposited energy with some track. Deposited energy near more than one track is shared in the manner most consistent with the energy deposition pattern expected from Monte Carlo studies. The direction of neutral tracks is also adjusted in this optimization procedure. The direction of charged tracks is taken as the center of the bump crystal. The second method for energy estimation, called ENER13, involves summing the energy of the bump crystal with its 12(11) neighbors. This sum, called E13, is corrected upward by $2.25 \%$ to compensate for lateral leakage outside the group of 13(12) crystals. A further correction based on the track's direction relative 
to the center of the bump crystal is also applied ${ }^{[4]}$ Because energy is not shared between two nearby tracks in the calculation of ENER13, it should only be used with well separated tracks to avoid double counting the energy in crystals which border two bumps. In this case, ENER13 gives a better energy resolution than ESORT for electromagnetically showering particles, and will be used throughout this analysis. Although ENER13 is a corrected E13, the term E13 will imply that the corrections have been applied.

The last step in the off-line analysis deals with the Time of Flight system (TOF) and is implemented by the routine TOFANL. Again, TOF information is not used in this thesis.

\subsection{Data Reduction and Compression}

Along with the large data samples obtained at DORIS came the practical problem of data handling. If the data on the $\sim 140 \Upsilon(1 \mathrm{~S})$ raw data tapes were not reduced in some manner, the number of analyzed tapes would be inconveniently large to efficiently extract physics. Several techniques have been employed to decrease the tape requirements while retaining information necessary for physics analyses. The first reduction technique the data encounters is a software trigger, called EOTAP, performed after the bumps stage in the off-line analysis. Each event must pass at least one of many EOTAP selection criterion!5] Each criterion corresponds to some desired physics analysis and loose cuts are invoked to select events that may be useful to that analysis. If an event fails all selection cuts, no further analysis is done and the event is rejected, with the exception that every tenth rejected event is kept for background studies. The only cut of importance for this study is the total energy requirement, that all events with greater than $1200 \mathrm{MeV}$ be kept. This requirement is $100 \%$ efficient for this analysis.

After the off-line analysis, events which pass EOTAP are written to tape. Because crystal ADC pulse heights are rarely needed after the crystal energies have been calculated, these pulse heights are dropped from the event record. 
Simple word packing is also done on some data. With EOTAP cuts and this simple word packing, the number of data tapes written is roughly a factor of two more than the number of input tapes. For inclusive analyses, this would entail spinning $\sim 250$ tapes for each pass through the data.

A more drastic approach to data compression is the use of the CRUSH format. CRUSH achieves about a 5 to 1 reduction in data storage requirements. This is done by dropping the tube chamber ADC and TDC pulse heights, which are not needed once tracking and/or tagging has been done, and by integerizing all data and storing it in bit patterns rather than in 32-bit words. The penalty for this dense data structure is that more CPU time is needed to read and write CRUSH tapes. Roughly $50 \%$ more CPU time is needed to write or read an event in CRUSH format, but because data analyses often require many more CPU cycles than these $\mathrm{I} / \mathrm{O}$ routines, the effective increase is usually much smaller than $50 \%$, depending on the analysis. Details about the CRUSH format can be found in Reference 1.

\subsection{Additional Data Processing}

Many important physical quantities are not calculated by the standard offline production code, but rather by the individual user's code. These quantities are continually recalculated each time a physics analysis is executed. The program SLED was developed to make many common calculations not in the standard production, and to have the results saved in the event record. SLED provided the user with all the commonly used topological variables such as thrust, sphericity, acoplanarity, etc. In addition SLED calls a pattern recognition routine called PIFIT. PIFIT is used to separate one and two photon connected regions and has been described in detail elsewhere[6] The important features will be summarized here as it is used to separate photons in this analysis.

For a given neutral connected region, PIFIT first assumes the region is consistent with one photon and adjusts the photon direction to maximize a likelihood 
function. This likelihood function is derived from Monte Carlo studies and gives the probability a photon could create the deposition pattern as a function of the photon direction. PIFIT then assumes the connected region is consistent with two photons and again maximizes the likelihood function by adjusting both the angles and energies of the two photons, constraining the sum of the photon energies to the connected region energy. PIFIT returns the mass of the two photons in this later case and the logarithm of the ratio of these two likelihoods. Because PIFIT is very CPU intensive, these quantities are also stored in the event record by SLED.

\subsection{Hadron Selection}

Each event passes through two software hadron selection routines in EOTAP. These routines, called NEWHAD and HJTHAD, efficiently select hadrons by rejecting QED, beam-gas and cosmic ray backgrounds. Although the detailed cuts for each routine differ, the selection criteria and program philosophy are quite similar. QED events are eliminated by requiring the events to have a minimum particle multiplicity and placing an upper bound on the energy deposition in the two most energetic connected regions. Beam-gas and cosmic backgrounds are rejected with cuts in event asymmetry and transverse momentum to the beam axis. A software flag for each selector is set in the event record if the respective routine identified the event as a hadron. This makes for easy hadron identification in later analyses.

\subsection{Monte Carlo Simulations}

Simulation of the physics events relevant to this analysis are used to estimate the detection efficiency for these processes. The event simulation is performed in three stages. In the first stage, the energy and direction of each particle in the primary vertex is generated. Each generated particle is allowed to decay through the appropriate decay channel. Each decay product is in turn allowed to decay 
until all particles in the final state are stable on the time scale needed to traverse the Crystal Ball detector.

The second stage traces each particle through the detector to simulate the energy deposition process. Electromagnetically showering particles are simulated with the Electron Gamma Shower (EGS) routines ${ }^{[7]}$ The geometry of the Crystal Ball detector, including the DORIS endcap configuration, the material between each crystal and the material between the hemispheres is modeled by this code. The pattern of energy deposition simulated by these routines has been compared with that found in real data; ${ }^{[4,6]}$ the two agree very well for the distributions used in this analysis. The High Energy Transport Code (HETC) ${ }^{[8]}$ is used to simulate charged pions and kaons. Comparisons sensitive to this code ${ }^{[0,10]}$ find the Monte Carlo in reasonable agreement with the data.

The third step in the event simulation involves adding the energy deposition from randomly selected DBM events, described in Chapter 2, with the output from the second step. This is done so that the simulated events also model the machine related backgrounds inherent in the data. More information about the Monte Carlo data sets used in this analysis can be found in Chapter 4. 
1) S. Lowe, Introduction to the CRUSH Format, Crystal Ball Off-line Workshop, SLAC, August 29-September 2, 1983.

2) M. Oreglia, Ph.D. thesis, "A Study of the Reactions $\psi^{\prime} \rightarrow \gamma \gamma \psi$," Stanford University, 1980, and SLAC Report 236 (unpublished).

3) R. Partridge, Crystal Ball Memo CB-6 (1979).

4) R. Lee, Ph.D. thesis, "Radiative Decays of the Psi Prime to All-Photon Final States," Stanford University, 1985. SLAC Report 282 (unpublished).

5) J. Gaiser, D. Gelphman, J. Irion, W. Lockman and J. Tomkins, Crystal Ball note 167, 1983.

6) R. Partridge, Ph.D. thesis, "A Study of the $\psi$ "(3770) Using the Crystal Ball Detector," California Institute of Technology, 1984 (unpublished).

7) R. Ford and W. Nelson, SLAC report number SLAC-0210, 1978.

8) T. Gabriel et al., Oak Ridge National Laboratory Report, ORNL/TM7123, January, 1981.

9) J. Gaiser, Ph.D. Thesis, "Charmonium Spectroscopy from Radiative Decays of the $J / \psi$ and $\psi^{\prime}$," Stanford University, 1982, SLAC Report 255 (unpublished).

10) W. J. Metzger, "Comparisons of Lund Monte Carlo with Crystal Ball Data On and Near the Upsilon", Nijmegen internal report HEN-278. 


\subsection{The $\Upsilon(1 S)$ Data Set}

The bulk of the $\Upsilon(1 S)$ data set was accumulated over 3 short runs taken in 1983, a much longer run collected in the Fall of 1984 and 2 runs taken in the Spring of 1986. Figure 4.1 shows the integrated luminosity per week for the $\Upsilon(1 \mathrm{~S})$ running with the total luminosity for each year indicated. The total data sample of $51.2 \mathrm{pb}^{-1}$ contains $14,079,847$ triggers and 599,414 selected hadrons. The small amount of luminosity collected in 1982 was obtained while debugging the DORIS II machine and the Crystal Ball detector. For this reason, and because of the high noise levels present in this data, the 1982 running is not used in this analysis.

Figure 4.2 shows the number of selected hadrons per selected Bhabha event for each $\Upsilon(1 S)$ run. The number of hadrons per Bhabha is proportional to $R_{\text {had }}$ defined as

$$
R_{\text {had }}=\frac{\sigma\left(e^{+} e^{-} \rightarrow \text { hadrons }\right)}{\sigma\left(e^{+} e^{-} \rightarrow \mu^{+} \mu^{-}\right)}
$$

Runs below 0.5 on this scale, indicated with a dashed line, are rejected to eliminate data taken significantly off resonance. The solid horizontal lines are the luminosity weighted averages for each year's running. The reduced hadronic cross section seen in 1983 indicates that, on average, the DORIS machine was running 


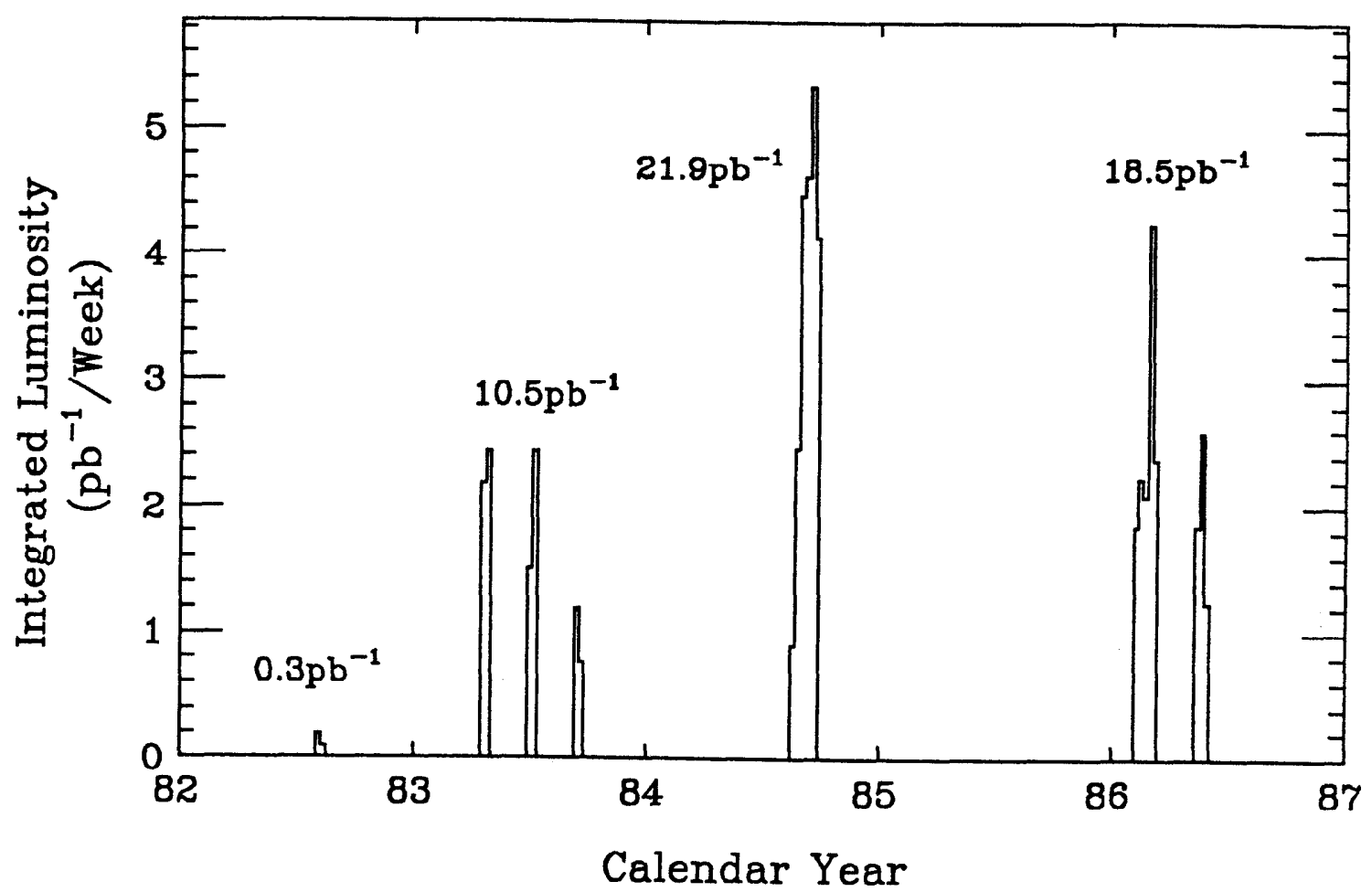

Fig. 4.1 The integrated luminosity collected on the $\Upsilon(1 \mathrm{~S})$ by the Crystal Ball. The total luminosity is $51.2 \pm 5.0 \mathrm{pb}^{-1}$. Subtotals for each year are also indicated.

slightly off the $\Upsilon(1 \mathrm{~S})$ resonance.

The total number of produced $\Upsilon(1 \mathrm{~S})$ events is calculated by subtracting the number of hadrons observed in off-resonance running from those observed from the on-resonance data, correcting for the hadron selection efficiency, the leptonic branching fractions of the $\Upsilon$, and background processes. This can be written as

$$
N_{\Upsilon(1 \mathrm{~S})}^{\text {prod }}=\frac{N^{o b s}(9.46)-N_{c o n t}^{o b s}(9.46)-N^{B . G .}(9.46)}{\epsilon_{\Upsilon(\mathrm{IS})}^{\text {had }} \cdot f^{\text {had }}+\epsilon_{\Upsilon(\mathrm{IS})}^{e^{+} e^{-}} \cdot f^{\mathrm{e}^{+} e^{-}}+\epsilon_{\Upsilon(\mathrm{IS})}^{\mu^{+} \mu^{-}} \cdot f^{\mu^{+} \mu^{-}}+\epsilon_{\Upsilon(\mathrm{IS})}^{\tau^{+} \tau^{-}} \cdot f^{\tau^{+} \tau^{-}}}
$$

where $N_{\Upsilon(1 \mathrm{~S})}^{\text {prod }}$ is the number of produced $\Upsilon(1 \mathrm{~S})$ events, $N^{\text {obs }}(9.46)$ is the number of hadrons observed in the on-resonance data, $N_{c o n t}^{o b s}(9.46)$ is the number of hadrons from continuum production at the $\Upsilon$ center of mass energy, $N^{B . G .}(9.46)$ counts 


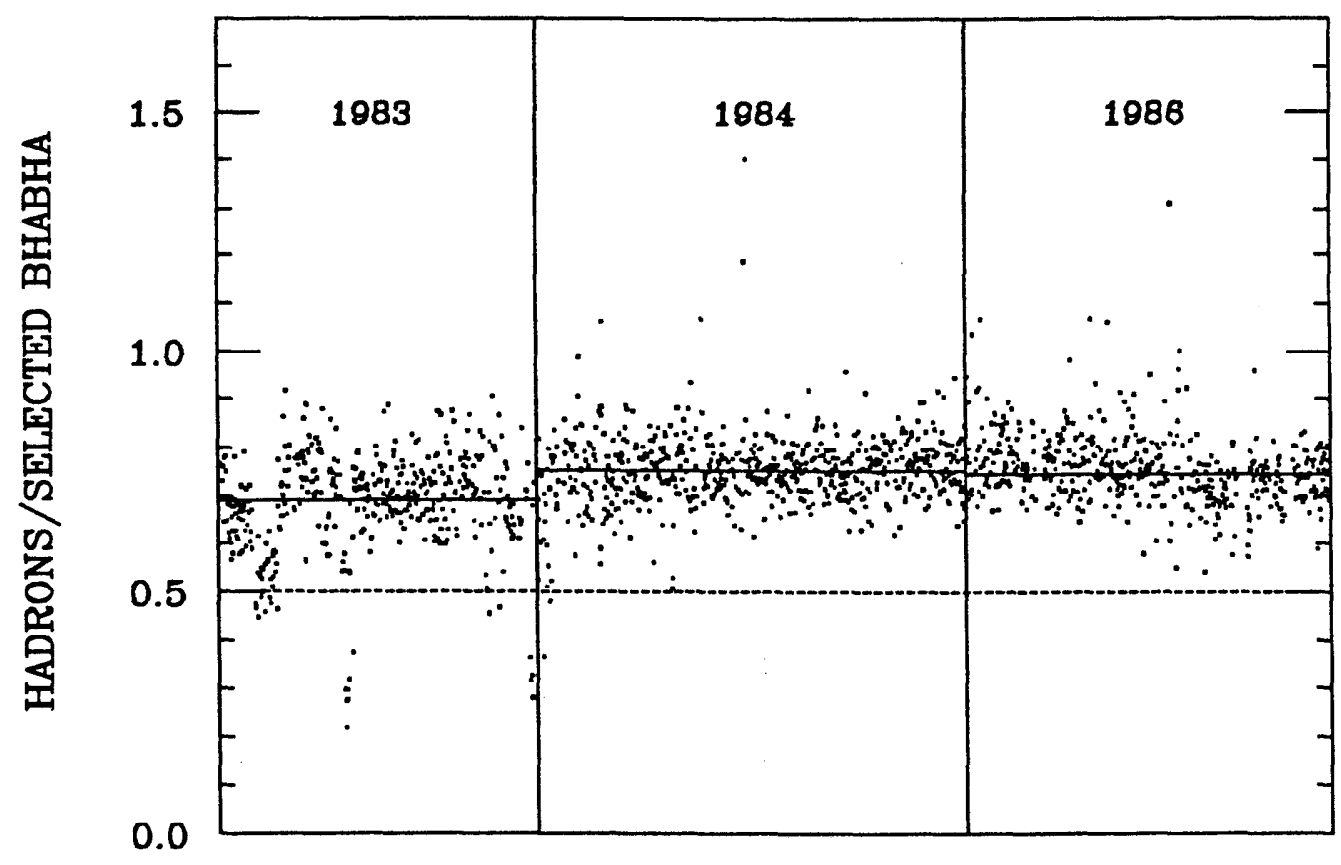

SEQUENTIAL 1S RUNS

Fig. 4.2 The number of selected hadrons per selected Bhabha. This ratio is proportional to $R_{\text {had }}$. Runs below 0.5 on this scale, shown as a dashed line, are significantly off resonance and are not used. The solid horizontal lines show the luminosity weighted averages for each year's running.

the number of beam-gas and beam-wall events which pass the hadron selection cuts, $\epsilon_{\Upsilon(1 S)}^{h a d}$ is the hadron selection efficiency, $\epsilon_{\Upsilon(1 S)}^{e^{+}}, \epsilon_{\Upsilon(1 S)}^{\mu^{+} \mu^{-}}$and $\epsilon_{\Upsilon(1 S)}^{\tau^{+} \tau^{-}}$are the efficiencies of the hadron selection routine on $\Upsilon(1 \mathrm{~S})$ leptonic decays, and fhad, $f^{e^{+} e^{-}}, f^{\mu^{+} \mu^{-}}$and $f^{\tau^{+} \tau^{-}}$are the branching fractions of the $\Upsilon(1 \mathrm{~S})$ to hadrons, $e^{+} e^{-}, \mu^{+} \mu^{-}$and $\tau^{+} \tau^{-}$, respectively. Note that $f^{\text {had }}+f^{e^{+} e^{-}}+f^{\mu^{+} \mu^{-}}+f^{\tau^{+} \tau^{-}}=1$. The quantity $N_{\text {cont }}^{o b s}(9.46)$ is obtained by counting the hadrons in off-resonance data and scaling for the $\frac{1}{S}$ dependence of the hadronic cross section and the luminosity. For data taken at $9.36 \mathrm{GeV}$ center of mass energy, this becomes

$$
N_{\text {cont }}^{\text {obs }}(9.46)=\left(N^{\text {obs }}(9.36)-N^{B . G .}(9.36)\right) \frac{L_{9.46}}{L_{9.36}} \frac{(9.36)^{2}}{(9.46)^{2}}
$$

where $N^{o b s}(9.36)$ is the number of observed hadrons in the off-resonance running 
at $9.36 \mathrm{GeV}$ and $N^{B . G .}(9.36)$ is the number of beam-gas and beam-wall events in this sample. The luminosities, $L_{9.36}$ and $L_{9.46}$, are obtained by counting large angle Bhabhas and scaling with the known cross section. Because the luminosities enter as a ratio, systematic scaling errors in these quantities cancel to lowest order.

The background from two photon production of hadrons is a negligible contribution here; the minimum total energy requirement of $2 \mathrm{GeV}$ and cuts on the transverse momentum eliminates essentially all such events. The continuum subtraction also statistically removes any remaining two photon contamination.

The hadron efficiency, $\epsilon_{\Upsilon(1 S)}^{\text {had }}$ is estimated from LUND Monte Carlo studies of hadronic decays and found to be $94 \pm 4 \%$. The only lepton pairs to have a non-zero efficiency are $\tau$ pairs. LUND Monte Carlo calculations based on $\tau$ decays of the $\Upsilon$ give $\epsilon_{\Upsilon(1 S)}^{\tau^{+} \tau^{-}}=18 \pm 3 \%$. The measured branching fractions of the $\Upsilon(1 S)$ to electron, muon and $\tau$ pairs $^{[1]}$ used in the calculation of the number of produced $\Upsilon(1 S)$ events, along with the number of hadrons and the corresponding luminosities for the on and off-resonance data, are shown in Table 4.1.

The beam-gas backgrounds, $N^{B . G .}(9.36)$ and $N^{B . G .}(9.46)$, are estimated from single-beam running. The number of hadronic events found in single-beam $e^{+}$ and $e^{-}$running are scaled to (pressure $\times$current $\times$live time) and summed to give the contamination in the $e^{+} e^{-}$colliding-beam data. The current and pressure are taken to be roughly equal for the single-beam and colliding-beam running. The large errors assigned to $N^{B . G .(9.36)}$ and $N^{B . G .}(9.46)$ reflect this assumption. The result of this scaling gives $N^{B \cdot G \cdot(9.36)}=1100 \pm 1100$ and $N^{B . G} \cdot(9.46)=15000 \pm 15000$. Substituting the values shown in Table 4.1 into equations $[4.1]$ and $[4.2]$ gives

$$
N_{\Upsilon(1 S)}^{p r o d}=(4.36 \pm 0.28) \times 10^{5}
$$

The error is dominated by systematic uncertainties in the Monte Carlo simulations and the beam-gas background subtraction. 


\begin{tabular}{|cc|cc|}
\hline$N^{\text {obs }}(9.46)$ & 596521 & $N^{\text {obs }}(9.36)$ & 51980 \\
\hline$N^{B . G .}(9.46)$ & $15000 \pm 15000$ & $N^{B . G .}(9.36)$ & $1100 \pm 1100$ \\
\hline$L_{9.46}$ & $50.7 \mathrm{pb}^{-1}$ & $L_{9.36}$ & $12.3 \mathrm{pb}^{-1}$ \\
\hline$B R\left(\Upsilon \rightarrow e^{+} e^{-}\right)$ & $2.8 \pm 0.3 \%$ & $\epsilon_{\Upsilon(1 \mathrm{~S})}^{\text {had }}$ & $0.94 \pm 0.04$ \\
\hline$B R\left(\Upsilon \rightarrow \mu^{+} \mu^{-}\right)$ & $2.8 \pm 0.2 \%$ & $\epsilon_{\Upsilon(1 \mathrm{~S})}^{e^{+}} \epsilon_{\Upsilon(1 \mathrm{~S})}^{\mu^{+} \mu^{-}}$ & 0.0 \\
\hline$B R\left(\Upsilon \rightarrow \tau^{+} \tau^{-}\right)$ & $3.2 \pm 0.4 \%$ & $\epsilon_{\Upsilon(1 \mathrm{~S})}^{\tau^{+} \tau^{-}}$ & $0.18 \pm 0.03$ \\
\hline
\end{tabular}

Table 4.1 Numerical quantities used in the calculation of produced hadrons.

Some machine and detector malfunctions occurred while collecting the $\Upsilon(1 \mathrm{~S})$ data set which require special treatment. During the 1984 run, about $15 \%$ of the crystal channels exhibited a non-linear response to energy deposition. This problem was studied in detail and is fully described in Appendix A. This problem caused an energy dependent inefficiency in the 1984 data which ranges from $10 \%-15 \%$ for photon energies between $500 \mathrm{MeV}$ and $2000 \mathrm{MeV}$. Figure A.13 in Appendix A shows the photon efficiency as a function of energy caused by the non-linear response. In addition, a malfunctioning ADC was used to convert the analog pulse heights from the tube chambers for roughly the first half of the 1984 running. This caused a non-linearity in the pulse-height response which was partially corrected for in the off-line software. Finally, as seen in Figure 4.2, the number of hadrons per Bhabha collected during the 1983 data taking was lower than that found in later running. As stated above, this is indicative of DORIS II running at energies slightly off the $\Upsilon$ (1S) resonance. The intrinsic width of a single beam at DORIS II is about $5.5 \mathrm{MeV}$. Because the hadronic cross section for the 1983 running was about $93 \%$ of the 1984 and 1986 running, the 1983 data was presumably taken about $\pm 3 \mathrm{MeV}$ off resonance, on average. 


\subsection{Determining Cuts Which Optimize Sensitivity}

Beginning with the $\Upsilon(1 S)$ data set defined in the previous section, a set of cuts is applied to enhance the radiative $\Upsilon$ events desired for this analysis. The underlying idea in selecting cuts is to reduce backgrounds while keeping the efficiency for radiative $\Upsilon$ decays as high as possible. This section is devoted to making this idea more quantitative and deriving a rule for determining cuts which maximize the sensitivity for the physical process studied here.

The terms "cut variable" or "cut parameter" describe the physical quantities being studied and can have a discrete or continuous distribution, e.g. "event multiplicity" or "total energy". The terms "cut value" or "cut requirement" indicate the range a cut variable must fall within in order to pass the cut.

The motivation for cutting background and keeping efficiency high is that in doing so, a real signal will have a higher statistical significance or, if no signal is within the detector's sensitivity, the resulting upper limit will be lower or more constraining. Typically, Monte Carlo simulations are used to model the decay of signal events in the detector. Although cuts tuned on such Monte Carlo events are acceptable, the cut values chosen will be ambiguous without knowledge of the background behavior; loose cuts that are $100 \%$ efficient for the Monte Carlo signal events may allow an overwhelming background into the final sample. Therefore, backgrounds must be well modeled before optimal cut values are chosen. If the major background sources are understood, a Monte Carlo simulation can also be used to model them. In the inclusive photon analysis presented here, however, the various background processes are not well enough understood to develop an accurate Monte Carlo simulation. Fortunately, the best source of background events is the data itself, provided the number of signal events is small compared to the total number of events. In this analysis, the number of signal events are

indeed small for the decays of interest. By using the actual data to model the backgrounds and a Monte Carlo simulation of the signal events, one can choose cut values which will maximize the sensitivity; the significance of any real signal 
will be the greatest or, if no such signals exist, the upper limit will be lowest. A rule that relates the sensitivity to the number of signal and background events is needed. As derived in Figure 4.3, the significance of any real signal which may be present is

$$
\sigma=\frac{S}{\sqrt{2 B+S}}=\frac{S}{\sqrt{2 B} \sqrt{1+\frac{1}{2}\left(\frac{S}{B}\right)}}
$$

assuming the total number of events is large enough to justify Gaussian statistics, where $S$ is the number of signal events and $B$ is the number of background events. The $\left(\frac{S}{B}\right)$ term in this equation is the signal to noise. If the signal is small compared to the background, and constants are dropped to derive a scaling relation then

$$
\sigma=\frac{S}{\sqrt{2 B} \sqrt{1+\frac{1}{2}\left(\frac{S}{B}\right)}} \simeq \frac{S}{\sqrt{2 B}} \propto \frac{S}{\sqrt{B}} \propto \frac{M}{\sqrt{B}}
$$

where the last term is useful when $M$ Monte Carlo events, used to simulate the signal process, pass the analysis cuts. Note that $M, B$ and $S$ are all functions of the cut values.

If no signals are apparent, an upper limit is derived by first calculating the detection error, $\Delta S$, for the signal process under study. The upper limit is then given by

$$
\text { U.L. }=\frac{k \cdot \Delta S}{N \epsilon}=\frac{k \cdot \sqrt{2 B}}{N \epsilon}
$$

where $\epsilon$ is the detection efficiency for the desired events, $k$ is a constant used to convert the $\Delta S$ to an upper limit, and $N$ is the number of $\Upsilon(1 S)$ events in the data sample. If $\mathrm{G}$ Monte Carlo events which simulate the signal are generated and $M$ events pass all the cuts then the efficiency is $\epsilon=M / G$. Dropping constants again leads to

$$
U . L .=\frac{k \cdot G \sqrt{2 B}}{M} \propto \frac{\sqrt{B}}{M} .
$$

Note $G$ can be dropped because it is not a function of the cut values. 


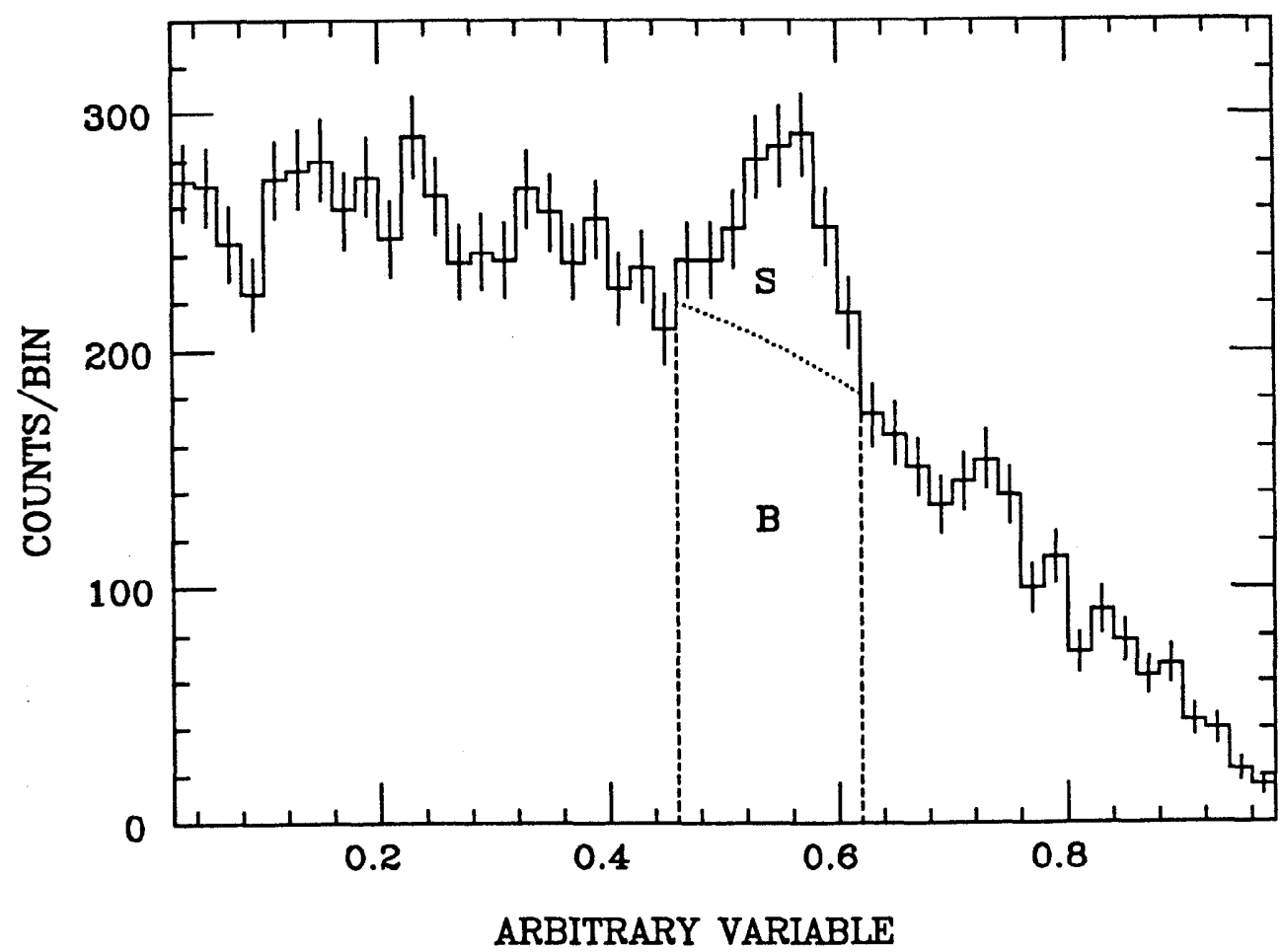

Fig. 4.9 A peak in an arbitrary spectrum. The significance of such a signal can be estimated by defining $B$, the number of background events, and $S$, the number of signal events. If the total number of events in the large bin, shown by dashed lines, is $\mathrm{N}$, and gaussian errors are assumed, then $S \pm \Delta S=(N \pm \Delta N)-(B \pm \Delta B)=$ $(N-B) \pm \sqrt{N+B}=(N-B) \pm \sqrt{S+2 B}$. The significance is then given by $\sigma=S / \sqrt{S}=S / \sqrt{S+2 B}$.

The last term in equations [4.4] and [4.5] indicate that the criteria used to maximize the significance of a small signal, if one exists, is equivalent to that which minimizes the upper limit when no signal is present. Thus, $R$ should be minimized as a function of cut values, where

$$
R \equiv \frac{\sqrt{B}}{M}
$$

to determine the most sensitive cuts. 


\subsection{The Cuts}

The ideas discussed in the last section are now used to determine the cut values for a variety of cut parameters. As stated above, the optimal cut values are those which minimize $R=\sqrt{B} / M$, where $\mathrm{B}$ is the number of background events and is calculated from the actual data, and $M$ is proportional to the efficiency which is simulated by Monte Carlo events. The Monte Carlo events in this case model the process

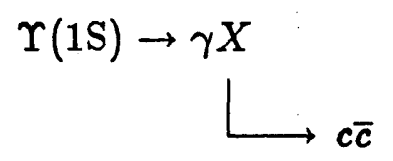

for photon energies ranging from $400 \mathrm{MeV}$ to about $3500 \mathrm{MeV}$ or equivalently for $\mathrm{X}$ mass between about $9051 \mathrm{MeV}$ and $4824 \mathrm{MeV}$, respectively. Approximately 2000 Monte Carlo events for each of 10 different photon energies are used for this optimization. The data used to model the background, B, consists of about $\frac{1}{2}$ of the 1986 data, taken uniformly over the sample. The only events used are those having at least one photon whose energy is within $4.0 \%$ of the nominal photon energy for one of the 10 Monte Carlo simulations. The 1986 data set was chosen because it is the most pristine; the 1983 data were presumably taken slightly offresonance and the 1984 data had the electronics problems discussed in section 4.1 and Appendix A. The 1983 and 1984 data sets were used in a similar analysis and the results were qualitatively the same.

The first cuts, listed in Table 4.2, are only used to define the initial data sample so no minimization of $R$ is done in this case. These cuts were derived from Monte Carlo studies; the tightest cuts which pass approximately $100 \%$ of the simulated events are used. 


\begin{tabular}{|c|c|}
\hline \multicolumn{2}{|c|}{ CUTS USED TO DEFINE DATA SET } \\
Cut Variable & Range to Pass Cut \\
\hline Hadron selector & NEWHAD or HJTHAD \\
\hline Total Multiplicity & $5 \leq N_{\text {mult }} \leq 22$ \\
\hline Total Energy in Ball & $2000 \mathrm{MeV} \leq E_{\text {total }} \leq 8500 \mathrm{MeV}$ \\
\hline
\end{tabular}

Table 4.2 Initial cuts used to define the $\Upsilon(1 \mathrm{~S})$ data set.

\subsubsection{Charge/Neutral Separation}

Because this analysis depends on the inclusive photon spectrum from the $\Upsilon(1 \mathrm{~S})$, the majority of the cuts involve defining clean photons. The first cut along this direction rejects particles which appear charged. As stated in Chapter 3, two methods exist for determining a particle's charge. The first method, offline production tagging, decides whether a particle is most probably charged or neutral. The second method, called CONTAG, assigns a probability that a given particle is charged. In order to calculate the photon efficiency using production tagging, some cut on the CONTAG probability, or some combination of these, pairs of particles in an event are matched and their invariant mass calculated. For example, Figure 4.4 shows the invariant mass plot for pairs of particles where both are tagged neutral by the production tagging. The number of $\pi^{0} \mathrm{~s}$ are estimated by fitting the histogram with a gaussian line shape over a polynomial background, with the mean and width fixed to nominal values. If $\epsilon_{N}$ is the efficiency for a truly neutral particle being called neutral by production tagging, then

$$
\epsilon_{N}=1-\frac{C \pm \Delta C}{2(N \pm \Delta N)+(C \pm \Delta C)}
$$

where $\mathrm{N}$ is the number of $\pi^{0} \mathrm{~s}$ in the neutral-neutral plot shown, and $\mathrm{C}$ is the number of $\pi^{0} \mathrm{~s}$ in a similar plot where the mass of charge-neutral pairs are plotted. 


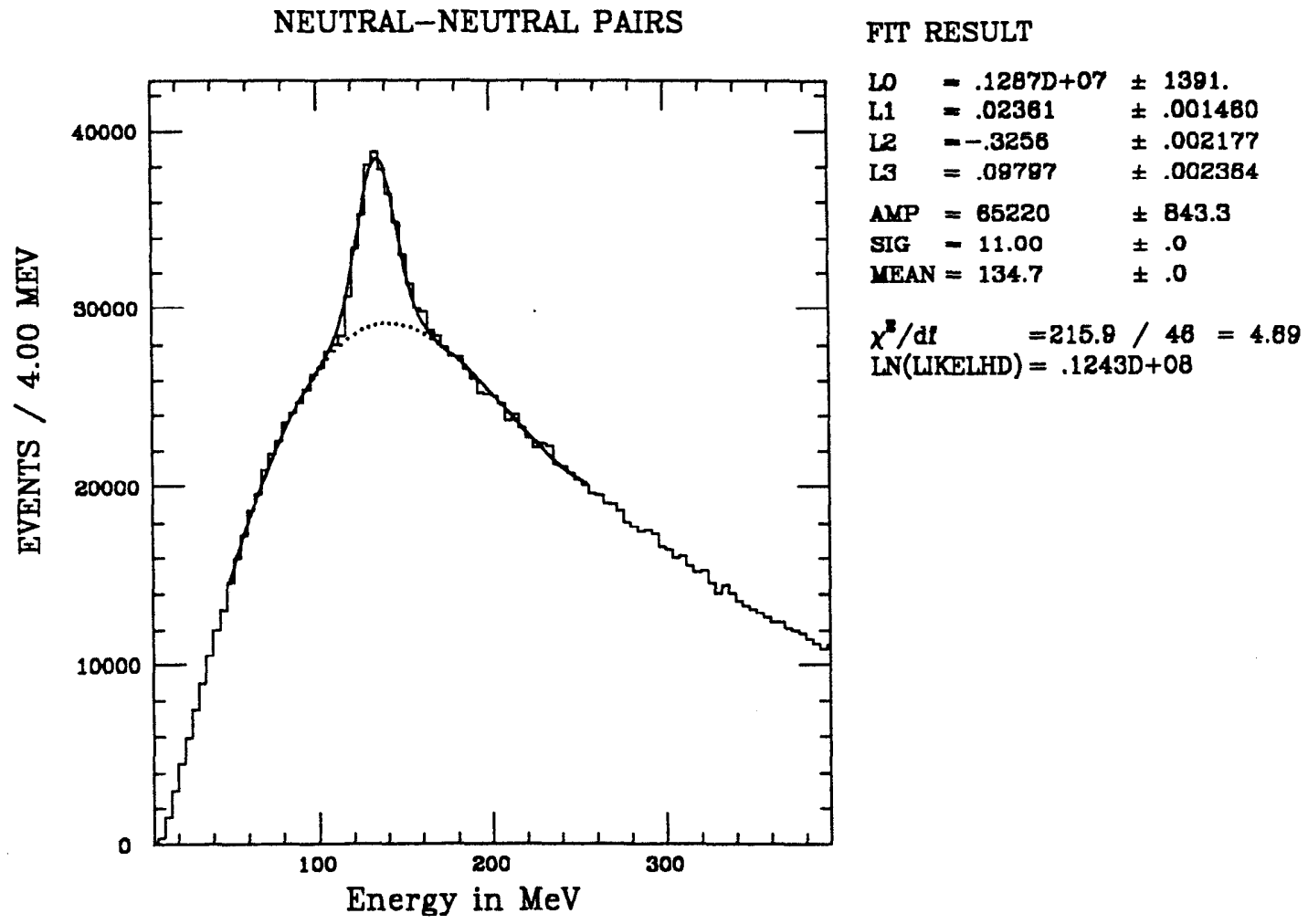

Fig. 4.4 The invariant mass plot for neutral-neutral combinations. A fit with fixed mean and width gives the total number of $\pi^{0} \mathrm{~s}$ in each case. This, along with a similar calculation for charge-neutral pairs, can be used to calculate the neutral efficiency.

Table 4.3 shows the efficiency for 17 possible charge/neutral requirements; production tagging alone, and 8 different cuts on the CONTAG charge probability both alone in conjunction with production tagging. In this latter case, if either CONTAG or production tagging finds the particle charged, it is considered charged. As shown earlier, it is not correct to pick the charge criterion with the highest efficiency; no cut would be $100 \%$ efficient but would also allow all charged particles to enter the photon sample. The quantity $R=\sqrt{B} / \epsilon_{N}$ should be minimized, where $\epsilon_{N}$ is calculated as in equation [4.7] above and $\mathrm{B}$ is modeled by the total number of neutral particles passing the particular charge/neutral requirement. The modeling of $\mathrm{B}$ by the total number of neutrals is appropriate because, for the same efficiency, a smaller number of particles assigned as neutral 


\begin{tabular}{|c|c|c|c|c|}
\hline CONTAG Cut & Prod. Tagging? & $\epsilon_{N} \pm \Delta \epsilon_{N}$ & $N_{\text {neutral }}$ & $R=\sqrt{N_{\text {neutral }}} / \epsilon_{N}$ \\
\hline none & yes & $95.15 \pm 0.70$ & 407612 & 6.70988 \\
\hline 0.00 & no & $86.86 \pm 0.48$ & 335654 & 6.67000 \\
\hline 0.05 & no & $90.86 \pm 0.74$ & 366660 & 6.66437 \\
\hline 0.10 & no & $93.28 \pm 0.75$ & 387712 & 6.67523 \\
\hline 0.15 & no & $94.90 \pm 0.70$ & 402585 & 6.68594 \\
\hline 0.20 & no & $95.73 \pm 0.70$ & 414886 & 6.72847 \\
\hline 0.25 & no & $95.77 \pm 0.68$ & 425485 & 6.81103 \\
\hline 0.30 & no & $95.65 \pm 0.57$ & 435449 & 6.89896 \\
\hline 0.40 & no & $96.20 \pm 0.66$ & 454637 & 7.00902 \\
\hline 0.00 & yes & $85.88 \pm 0.79$ & 325730 & 6.64564 \\
\hline 0.05 & yes & $89.45 \pm 0.75$ & 350719 & 6.62063 \\
\hline 0.10 & yes & $91.71 \pm 0.73$ & 365033 & 6.58794 \\
\hline 0.15 & yes & $92.92 \pm 0.72$ & 373512 & $6.57723(*)$ \\
\hline 0.20 & yes & $93.47 \pm 0.72$ & 379228 & 6.58837 \\
\hline 0.25 & yes & $93.80 \pm 0.71$ & 383495 & 6.60202 \\
\hline 0.30 & yes & $94.01 \pm 0.73$ & 386813 & 6.61571 \\
\hline 0.40 & yes & $94.48 \pm 0.70$ & 391855 & 6.62556 \\
\hline
\end{tabular}

Table 4.9 The results of several possible charge criteria on the minimization of $R$. The line in the right column labeled with an asterisk $(*)$ corresponds to the charge criteria giving the minimum $R$. Thus, throughout this analysis, charged particles are defined to be those having either a CONTAG probability greater than 0.15 or which are considered charged by production tagging. 
implies a smaller charge particle contamination. Table 4.3 lists the number of neutrals and $R$ for each combination. The minimum value of $R$ occurs when the CONTAG probability is greater than 0.15 and production tagging is used. Thus, throughout this analysis, charged particles are defined as tracks having either a CONTAG probability greater than 0.15 or being tagged charged by the production software; all other tracks are considered neutral.

\subsubsection{Photon Pattern Cuts}

Photon pattern cuts are used to separate quality photons from backgrounds resulting from the interaction of hadrons in the detector, overlapping particles, shower split-offs, and other sources. Several parameters are traditionally used as cut variables for Crystal Ball analyses. These are the ratios $E 1 / E 4, E 4 / E 13$, $E 2 / E 4, E 13 / E_{C R}$ where $E 1, E 2, E 4$ and $E 13$ are the geometrical energy sums defined in Chapter 2 and $E_{C R}$ is the energy sum for all crystals in the photon's connected region. Another common cut parameter is the log-likelihood difference from PIFIT, termed LLD, as defined in Chapter 3. The optimal cuts as defined above are derived for each of these 5 parameters as a function of photon energy. Again, the Monte Carlo simulations used here model the decay shown in equation [4.6] for 10 different photon energies, and the corresponding data sample used to model the background has at least one photon with energy within $4 \%$ of one of these values.

Figure 4.5 shows the result of minimizing $\mathrm{R}$ for the variable $E 1 / E 4$ for 3 different photon energies. The solid histogram shows the Monte Carlo distribution and the dotted histogram shows the data. It is important to note that for the Monte Carlo distributions, the candidate photon is required to be the prompt photon in the simulated decay $\Upsilon \rightarrow \gamma X$, while the data distributions correspond to any neutral track inside the proper energy bin. The value of $R$ is calculated for all combinations of lower and upper cut values for each plot. The vertical dashed lines indicate the cut values which minimize $R$ in each case. Note that 

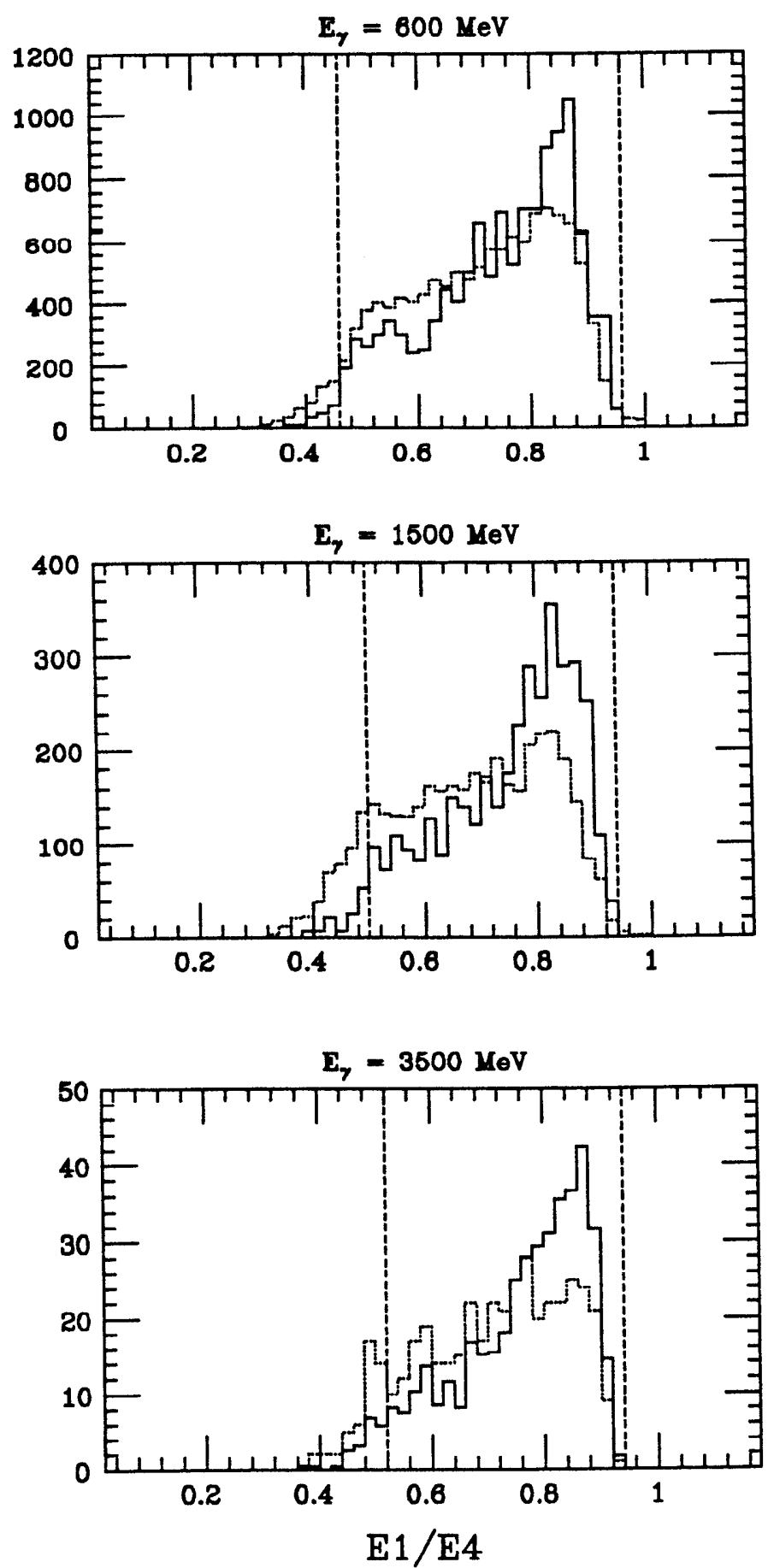

Fig. 4.5 The result of minimizing $R$ for the variable $E 1 / E 4$ shown for 3 different photon energies. The solid histogram is the Monte Carlo distribution and the dotted histogram is from the small data sample. The vertical dashed lines show the optimal cuts. 


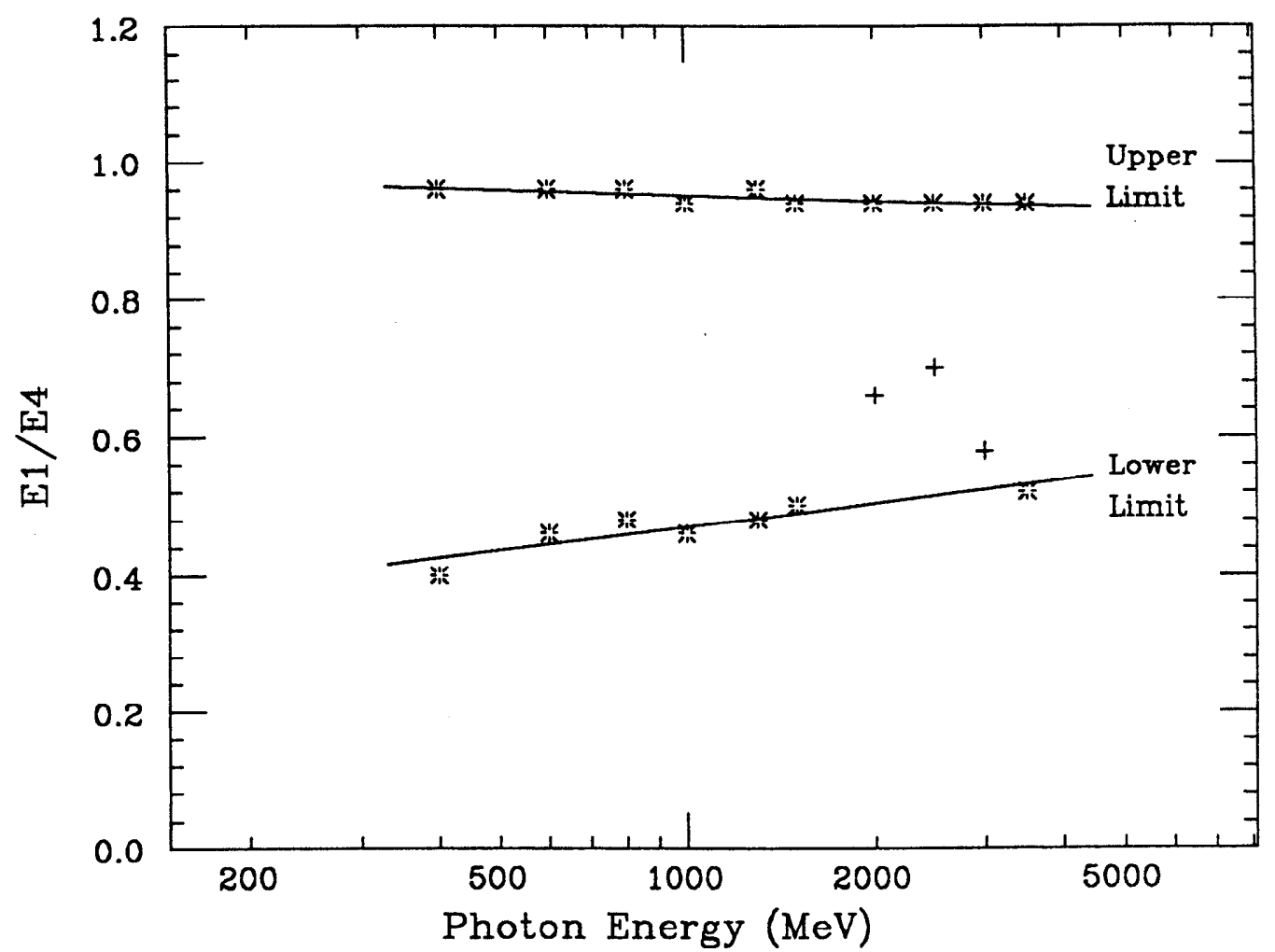

Fig. 4.6 The results from minimizing $R$ for $E 1 / E 4$ for all 10 photon energies. The straight-line fits represent the actual cuts used. The points labeled with crosses are not used in the fit to reduce possible systematic errors.

possible cut values are quantized because they must lie between bins. Figure 4.6 summarizes the results from such calculations for all 10 photon energies. To get a better behaved cut as a function of energy, the points for both the upper and lower cut values are fit to straight lines. The resulting fits are also plotted in Figure 4.6.

The three points labeled by crosses in the lower limit case are not used in the fit. These points deviate from the others because the Monte Carlo distributions and the data distributions differ significantly. This is illustrated by the distributions corresponding to the middle crossed point at $2500 \mathrm{MeV}$ and is shown in Figure 4.7. Because the data distribution corresponds to any neutral track while the Monte Carlo distribution corresponds only to the prompt photon from the $\Upsilon$ 


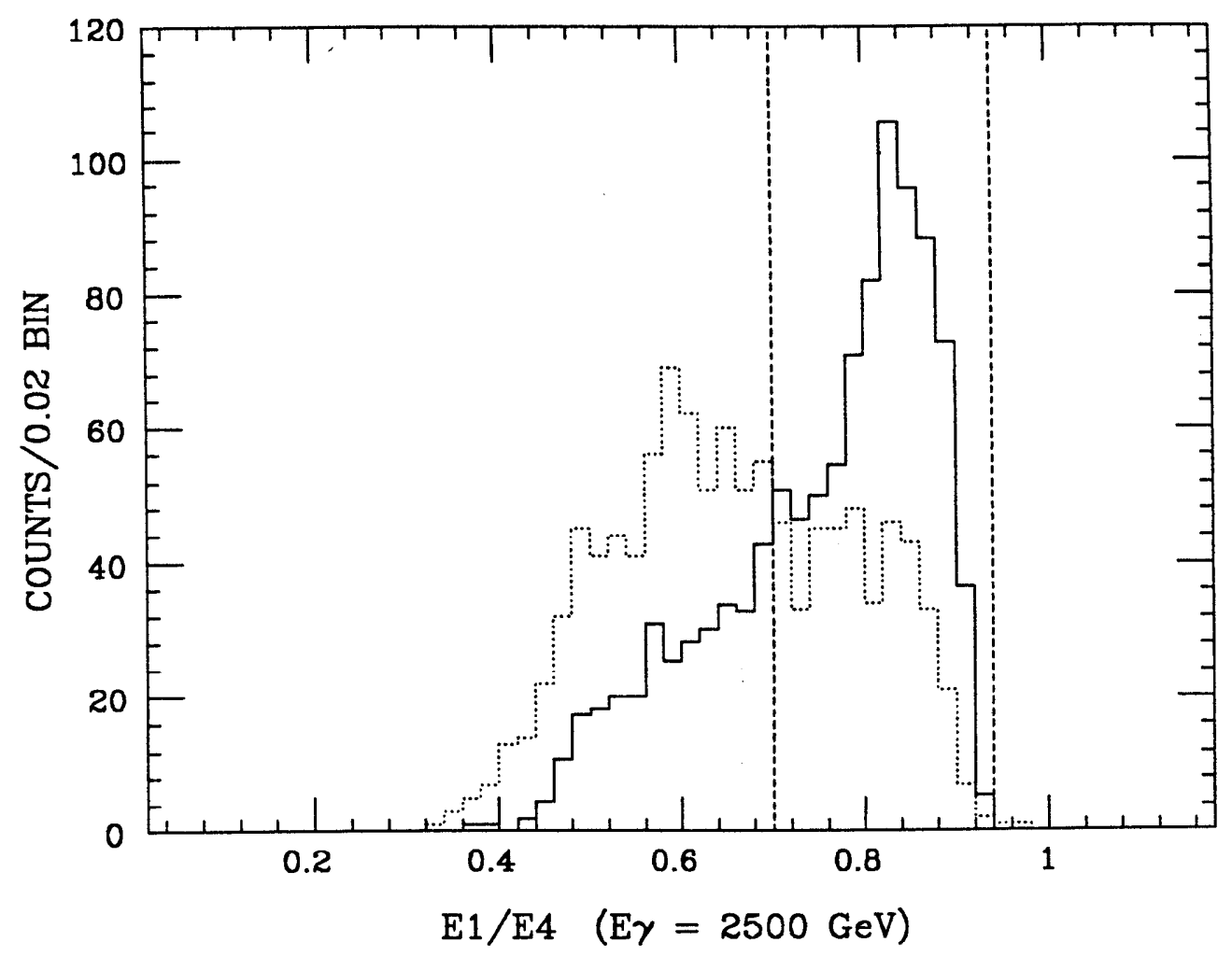

Fig. 4.7 The result of minimizing $R$ for $E 1 / E 4$ with photon energy of $2500 \mathrm{GeV}$. This shows the difference in the Monte Carlo and data distributions. The difference is due to the requirement that the Monte Carlo distribution correspond to the simulated prompt photon while no such requirement can be made with the data.

decay, the data distribution can have a large contamination. In this case, overlapping photons from $\pi^{0}$ decays and energy deposition from interacting hadrons which appear as a single track by the Crystal Ball software, enter the data distribution. The best cut in this case is that shown in Figure 4.7, and the overall, energy-dependent cut shown in Figure 4.6 should somehow incorporate the three crossed points. However, such a cut would not be a monotonic function of energy and could produce a systematically biased result. For this reason, these points were not used in the straight-line fit; the resulting sensitivity may be lower but the efficiency is higher and the systematic errors will not be as great. This approach is also used for other cut variables and always in a direction that softens 


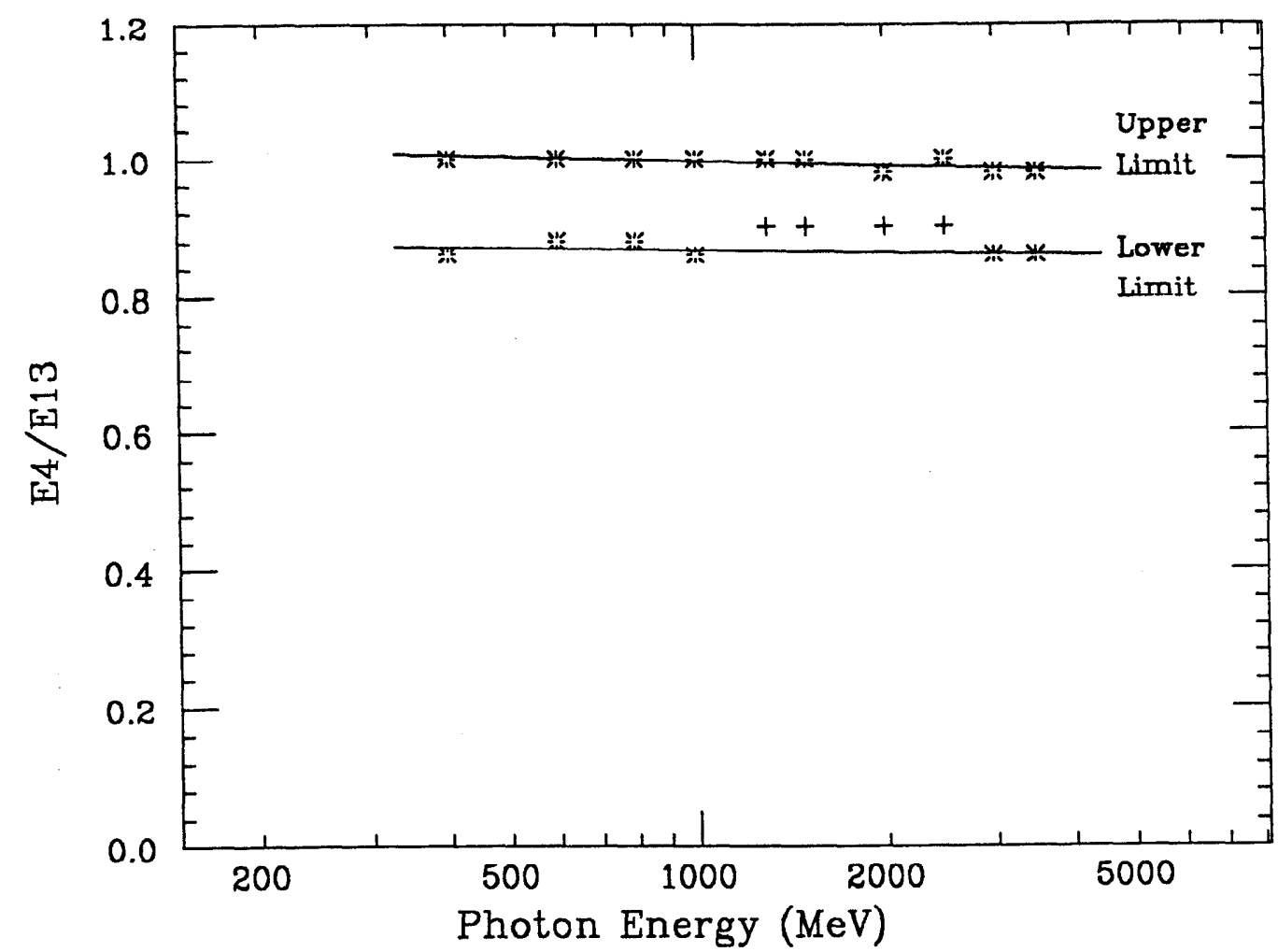

Fig. 4.8 The results from minimizing $R$ for $E 4 / E 13$. The straight-line fits represent the actual cuts used. As in Figure 4.6, the points labeled with crosses are not used in the fit

the cut on the data.

The next cut for which $R$ is minimized is $E 4 / E 13$. The analysis of this cut is analogous to the $E 1 / E 4$ case. Figure 4.8 shows the upper and lower cut boundaries for each of the photon energies where $R$ is minimized. Here again, a few points indicate a stronger cut should be used than the surrounding points, and are not included in the straight-line fits in order to reduce systematic errors.

The next cut attempted is the ratio E2/E4. Figure 4.9 shows the optimal cuts for 3 different photon energies. These plots show that this cut has little discriminating power at this stage of the analysis; distributions for the Monte Carlo and data are too similar. For this reason, no cut on this variable is used. This is also the situation for the variable $E 13 / E_{C R}$. Figure 4.10 shows the 

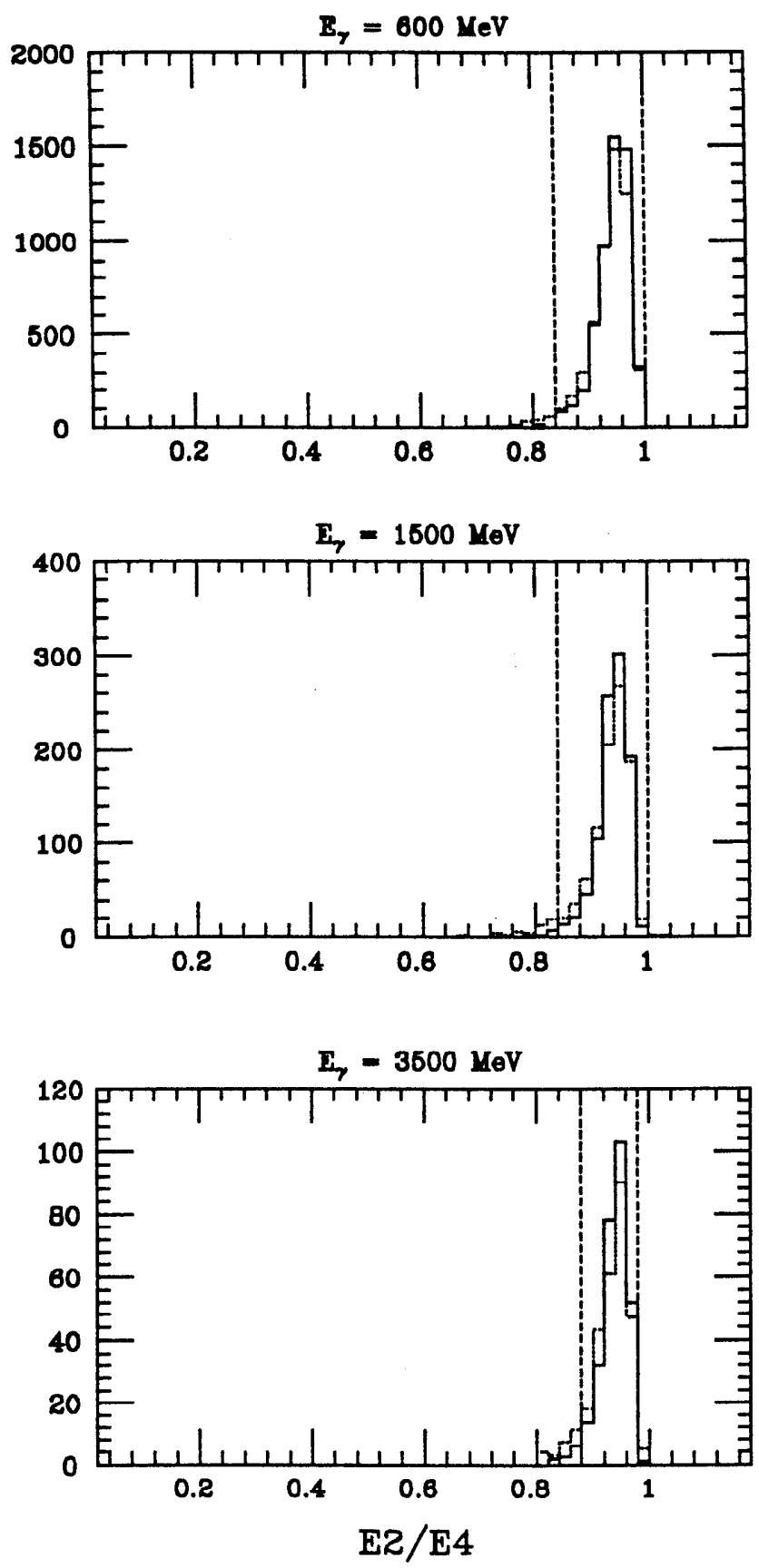

Fig. 4.9 The result of minimizing $R$ for the variable $E 2 / E 4$ shown for 3 different photon energies. The solid histogram is the Monte Carlo distribution and the dotted histogram is from the small data sample. The vertical dashed lines show the optimal cuts. Because the Monte Carlo events and data have such similar distributions, this cut has a negligible effect, and is not used in this analysis. 

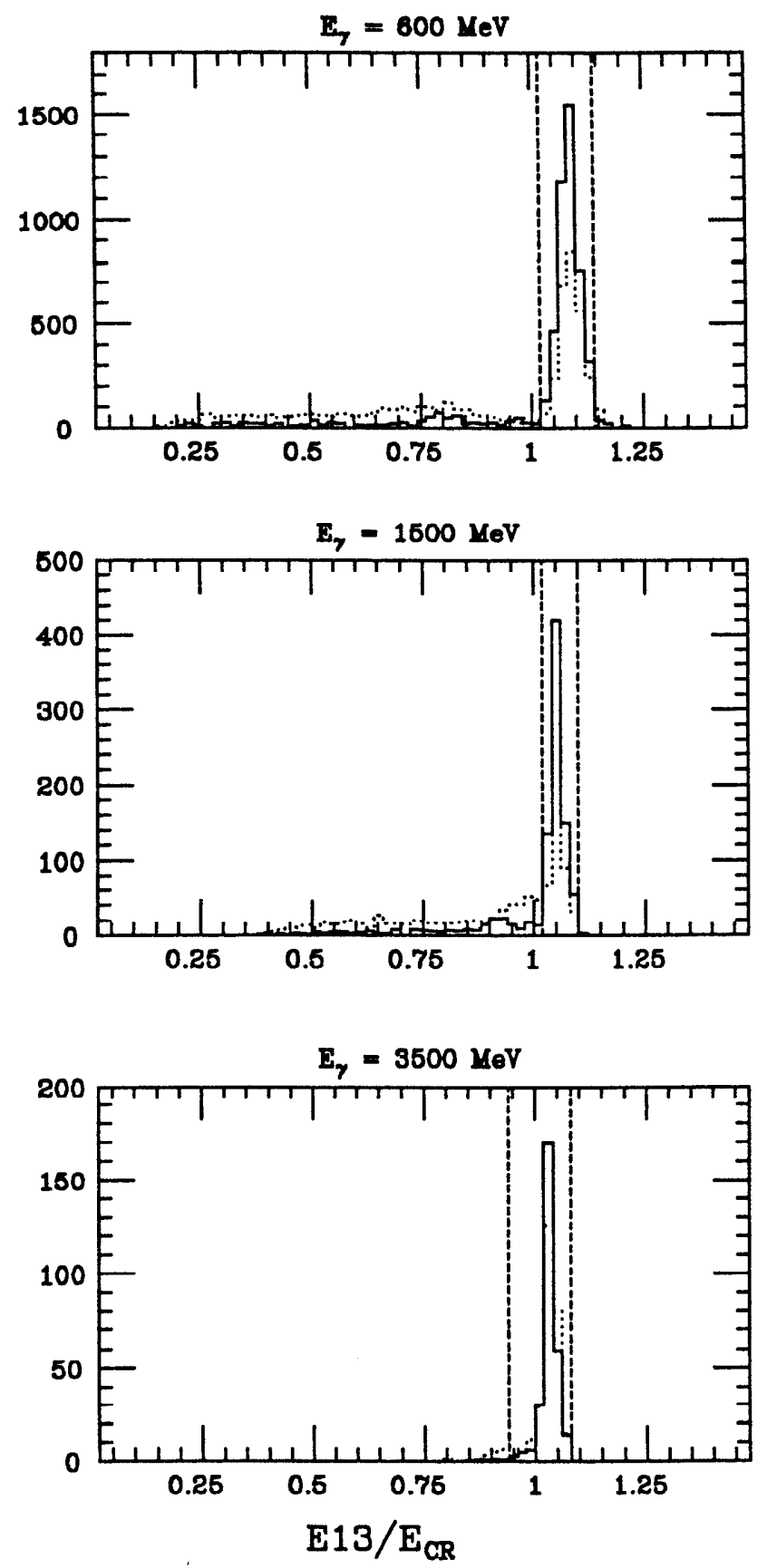

Fig. 4.10 The result of minimizing $R$ for the variable $E 13 / E_{C R}$ shown for 3 different photon energies. The solid histogram is the Monte Carlo distribution and the dotted histogram is from the small data sample. The vertical dashed lines show the optimal cuts. Because the Monte Carlo events and data have such similar distributions, this cut has a negligible effect, and is not used in this analysis. 


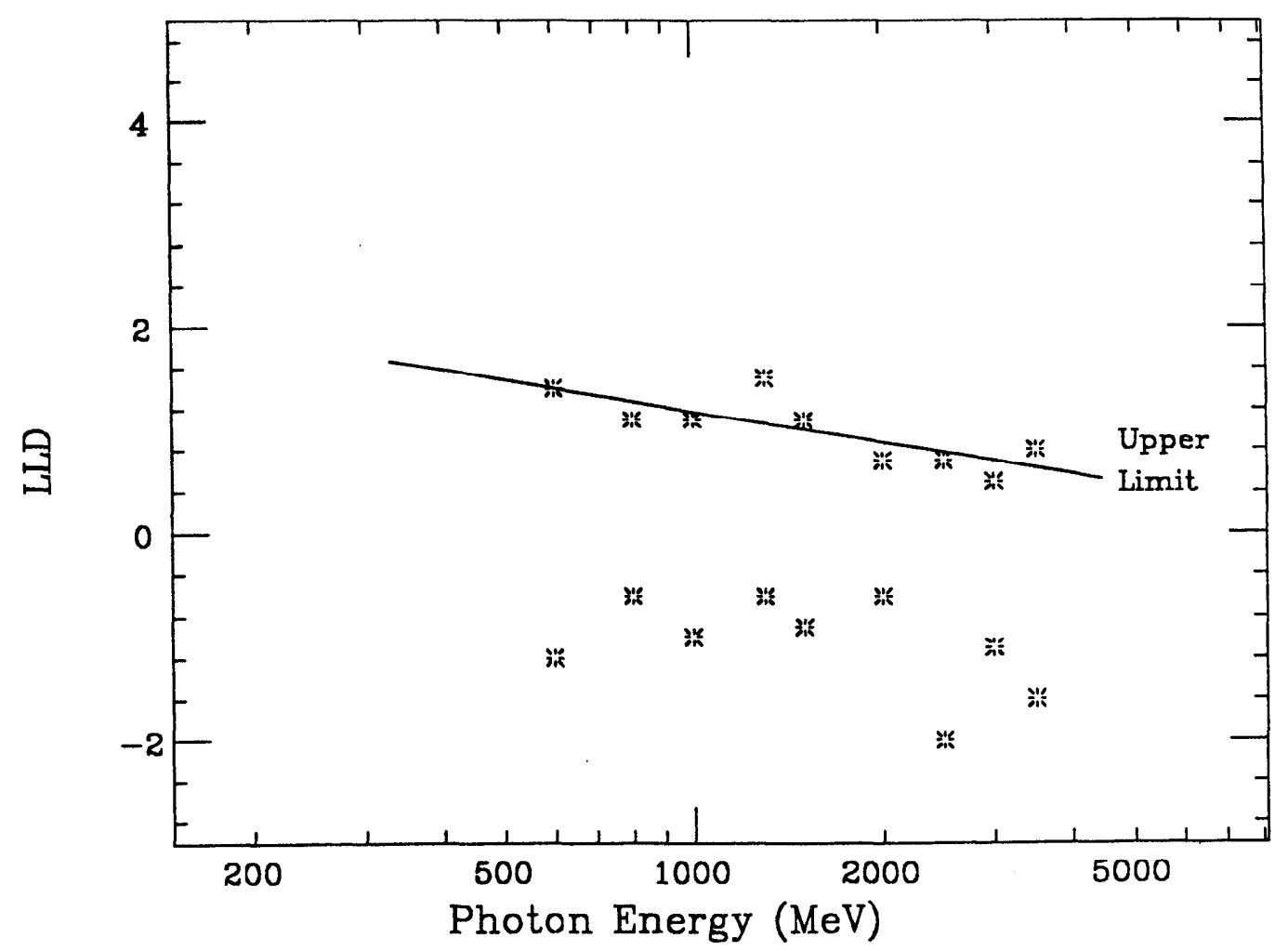

Fig. 4.11 The results from minimizing LLD from PIFIT for 9 photon energies. The straight-line fit for the upper cut limit represents the actual cut used. The lower points are not used because they are dictated by small fluctuations in the tail of the distributions and produce a negligible effect (see Figure 4.12).

Monte Carlo and data distributions along with the optimal cuts. Because the discriminating power is also small for this variable, no cut on this parameter is used. Note that this does not necessarily mean these cut variables are not useful; if these cuts were placed first, they might have a much greater discriminating power.

The final photon pattern cut attempted is LLD from the routine PIFIT. Figure 4.11 shows the results from minimizing $R$ for all photon energies. The point at $400 \mathrm{MeV}$ is not used because PIFIT does not work well at this energy or for lower energies. The points corresponding to the lower cut limit show a scattered behavior. The plots in Figure 4.12 indicate that this scattering is due 

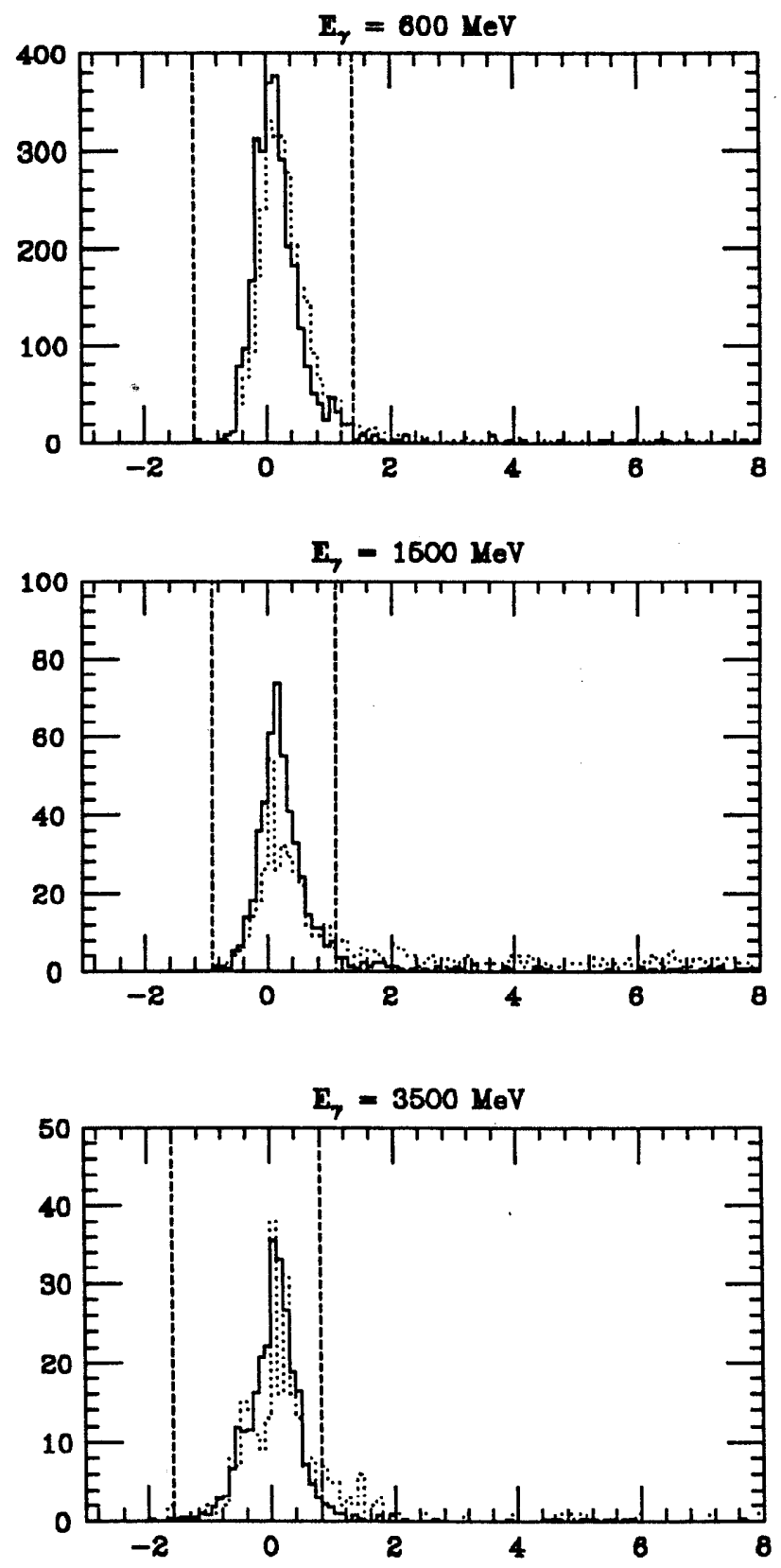

Lل

Fig. 4.12 The result of minimizing $R$ for LLD from PIFIT shown for 3 different photon energies. The solid histogram is the Monte Carlo distribution and the dotted histogram is from the small data sample. The vertical dashed lines show the optimal cuts. Note the lower cut limit is governed by small fluctuations in the lower tail of the distribution. Because of this and because the lower cut has a negligible effect, it is not used in this analysis. 
to random counts in the lower tails of the distributions. Because of this behavior and because the lower cut produces a negligible effect, only the upper cut is used.

\subsubsection{Particle Overlap Cut}

An important cut used to isolate photons from other neighboring particles is the overlap cut. This is simply a lower limit on the minimum angle between the photon in question and any other detected particle. The upper plot in Figure 4.13 shows the minimization of $R$ for $2000 \mathrm{MeV}$ photons while the lower plot summarizes the results for the various different photon energies. The slope of the straight-line fit shows that for high-energy photons a very loose cut is used. This is expected because the recoil system is highly boosted away from the photon direction so that there is little probability of another particle overlapping the photon. For low energy photons, there is a high probability of an overlap so the cut becomes tighter.

\subsubsection{Low-Energy $\pi^{0}$ Subtraction}

Intermediate-energy $\pi^{0} \mathrm{~s}$, in the $500 \mathrm{MeV}$ to $2500 \mathrm{MeV}$ range, have already been cut with the $E 4 / E 13$ and LLD cuts. The two decay photons from highenergy $\pi^{0} \mathrm{~s}$, above about $2500 \mathrm{MeV}$, are so close that they cannot be resolved and appear as a single photon; a small background which cannot be easily eliminated. Photons from low-energy $\pi^{0} \mathrm{~s}$, however, can be subtracted. This is accomplished by pairing the photon candidate with other neutral particles in the event and calculating the invariant mass of the pair. The photon candidate is rejected if the mass of any pair is sufficiently close to the $\pi^{0}$ mass.

This-low energy $\pi^{0}$ subtraction depends on several criteria such as the requirements to select the other neutrals paired with the photon or the mass range of the pair which constitutes a $\pi^{0}$. The method used here is similar to that used to select a charge criteria; to try several possible definitions of $\pi^{0}$ candidates and to choose the one giving the best value of $R$. There are 4 possible criteria 

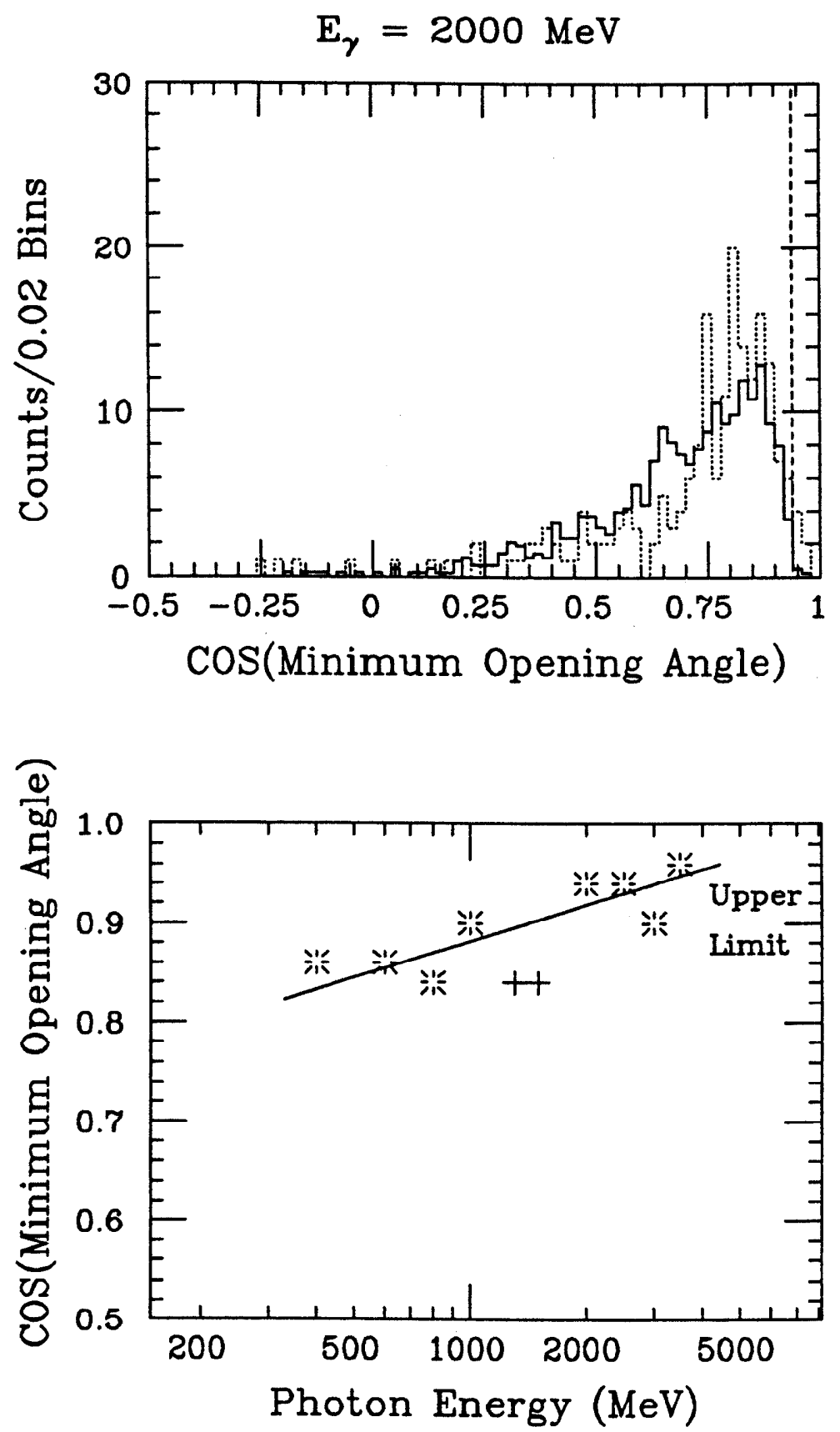

Fig. 4.13 The results of minimizing $R$ for the minimum opening angle cut. The top plot shows an example of the minimization of $R$ for a photon energy of $2000 \mathrm{MeV}$. The bottom plot summarizes results from all 9 photon energies. As in Figure 4.6, the points labeled with crosses are not used in the fit. 
for the neutral to be paired with the candidate photon. Each neutral particle to be paired with the photon must have an energy of at least $20 \mathrm{MeV}$ and its angle from the beam pipe, $\theta_{Z}$, must satisfy $\left|\cos \theta_{Z}\right| \leq 0.85$. In addition to these criteria, each of these particles may pass up to 3 progressively tighter cuts:

1) $0.40 \leq E 1 / E 4 \leq 0.98$ and $0.70 \leq E 4 / E 13 \leq 0.995$

2) $0.50 \leq E 1 / E 4 \leq 0.96$ and $0.78 \leq E 4 / E 13 \leq 0.985$

3) $\left|\cos \theta_{Z}\right| \leq 0.80$,

making 4 possibilities in all.

The invariant mass of the photon and the other neutral to be considered must satisfy the relation

$$
\left|\frac{\left(M_{\text {pair }}-M_{\pi^{0}}\right)}{M_{\pi^{0}}}\right| \leq K
$$

where 3 possible values of $K$ are tested; $K=0.22,0.20$ and 0.18 . For each of the 4 possible cuts on the paired particle and the 3 possible values of $K$, and for each of the 9 photon energies, $R$ is calculated. The value of $R$ is also calculated for no $\pi^{0}$ subtraction and for a global $\pi^{0}$ subtraction implemented by the routine PHYSAC. This routine looks at all possible pairings of candidate photons and finds the best pairing for the event by maximizing a global likelihood.

The results of the $R$ calculations show that, for photon energies higher than 1500-2000 MeV, no cut gives the lowest value of $R$. This is expected, because the two photons from the $\pi^{0}$ decay are more likely to be folded over and appear as a single particle in the Crystal Ball detector at such energies. For the lower photon energies, the set of cuts labeled 2) above is clearly favored, with $K$ being either 0.20 or 0.18 , depending on the photon energy. Because the value of $R$ is almost identical in these two cases, $K=0.20$ is chosen. Note that this cut is applied even for high energy photons to avoid a discrete change in the cuts at a particular energy. This is reasonable because the efficiency remains high for this cut at higher photon energies. 


\begin{tabular}{|c|c|}
\hline \multicolumn{2}{|c|}{ FINAL SET OF ANALYSIS CUTS } \\
Cut Variable & Range to Pass Cut \\
\hline Hadron selector & $5 \leq N_{\text {mult }} \leq 22$ \\
\hline Total Multiplicity & $2000 \mathrm{MeV} \leq E_{\text {total }} \leq 8500 \mathrm{MeV}$ \\
\hline Total Energy in Ball & Neutral by Production Tagging \\
\hline Photon Selection & CONTAG Bump Probability $\leq 0.15$ \\
& $\left|\cos \theta_{Z}\right| \leq 0.90$ \\
& $0.13136-0.04896 X \leq E 1 / E 4 \leq 1.02893-0.01123 X$ \\
& $0.90203-0.00503 X \leq E 4 / E 13 \leq 1.06149-0.00937 X$ \\
& $L L D \leq 4.20414-0.43810 X$ \\
\hline Overlap Cut & $\cos \theta_{A B} \leq 0.51470-0.05306 X$ \\
\hline$\pi^{0}$ Subtraction & $\left|M_{\text {pair }}-M_{\pi^{0}}\right| / M_{\pi^{0}} \leq 0.20$ \\
\hline
\end{tabular}

Table 4.4 The final set of cuts used in this analysis. $X$ is the natural $\log$ of the photon energy, $\theta_{A B}$ is the angle between the photon and the nearest particle, and $M_{\text {pair }}$ is defined in the text.

\subsubsection{Notes on Final Cuts}

This completes the set of cut parameters used in this analysis. Several comments should be made here. First, the set of cut variables considered above are based on historical prejudice; they are found in most Crystal Ball analyses. Improved cuts, based on parameters which better separate potential signal events from background events, surely exist but whether the increase in sensitivity is great enough to warrant the large effort in searching for such variables is questionable. Because the cut parameters are highly correlated, recalculating the cut values for the first cut after the other cuts are applied might be justified. In other words, because $R$ is a function of many cut variables, it should be mini- 
mized globally in a many-dimensional space, rather than only once in each single dimension. Minimizing $R$ iteratively for each cut variable was attempted but resulted in essentially identical cuts to those shown here with only one iteration.

The final set of cuts are shown in Table 4.4. In comparison to other similar analyses, these cuts are very loose. The background here is higher than would otherwise be expected for an analysis such as this, but the efficiency is also higher. Typically, Crystal Ball analyses utilize cuts which increase the signal to noise because signals look more obvious ${ }^{[2]}$ However, the significance of any signal, along with the sensitivity, does not usually increase with such cuts.

\subsection{The $\Upsilon(1 S)$ Photon Spectrum}

Applying the cuts derived in the last section to the $\Upsilon(1 S)$ data set leads to the sequence of plots shown in Figure 4.14. The last plot in Figure 4.14 is the final $\Upsilon(1 S)$ photon spectrum and shown in Figure 4.15 at an enlarged scale. There are no obvious indications of a narrow state resulting from the decay $\Upsilon(1 \mathrm{~S}) \rightarrow \gamma X$. Thus, the result of this analysis will be an upper limit on the branching ratio for this process.

There is however, an obvious, broad enhancement of photons in Figure 4.15 from 2 to $5 \mathrm{GeV}$. Direct photons from the $\Upsilon(1 \mathrm{~S})$ via the decay $\Upsilon \rightarrow \gamma g g$ contribute to this plot, but without a full analysis, including subtraction of nonresonant hadron production, the electromagnetic decay $\Upsilon(1 \mathrm{~S}) \rightarrow q \bar{q}$, photons from high energy $\pi^{0}$ decays, and feeddown from QED processes, it is difficult to determine what fraction of this enhancement is from $\Upsilon \rightarrow \gamma g g$. Comparison with measurements of the direct $\Upsilon(1 S)$ photon spectrum from the CUSB $^{[3]}$ and $\mathrm{CLEO}^{[4]}$ collaborations indicates that roughly $\frac{1}{2}$ or more of this enhancement is due to the $\Upsilon(1 \mathrm{~S}) \rightarrow \gamma g g$ decay mode. Figure 4.16 shows the inclusive photon spectrum plotted as a function of $x=E_{\gamma} / E_{\text {beam }}$. 

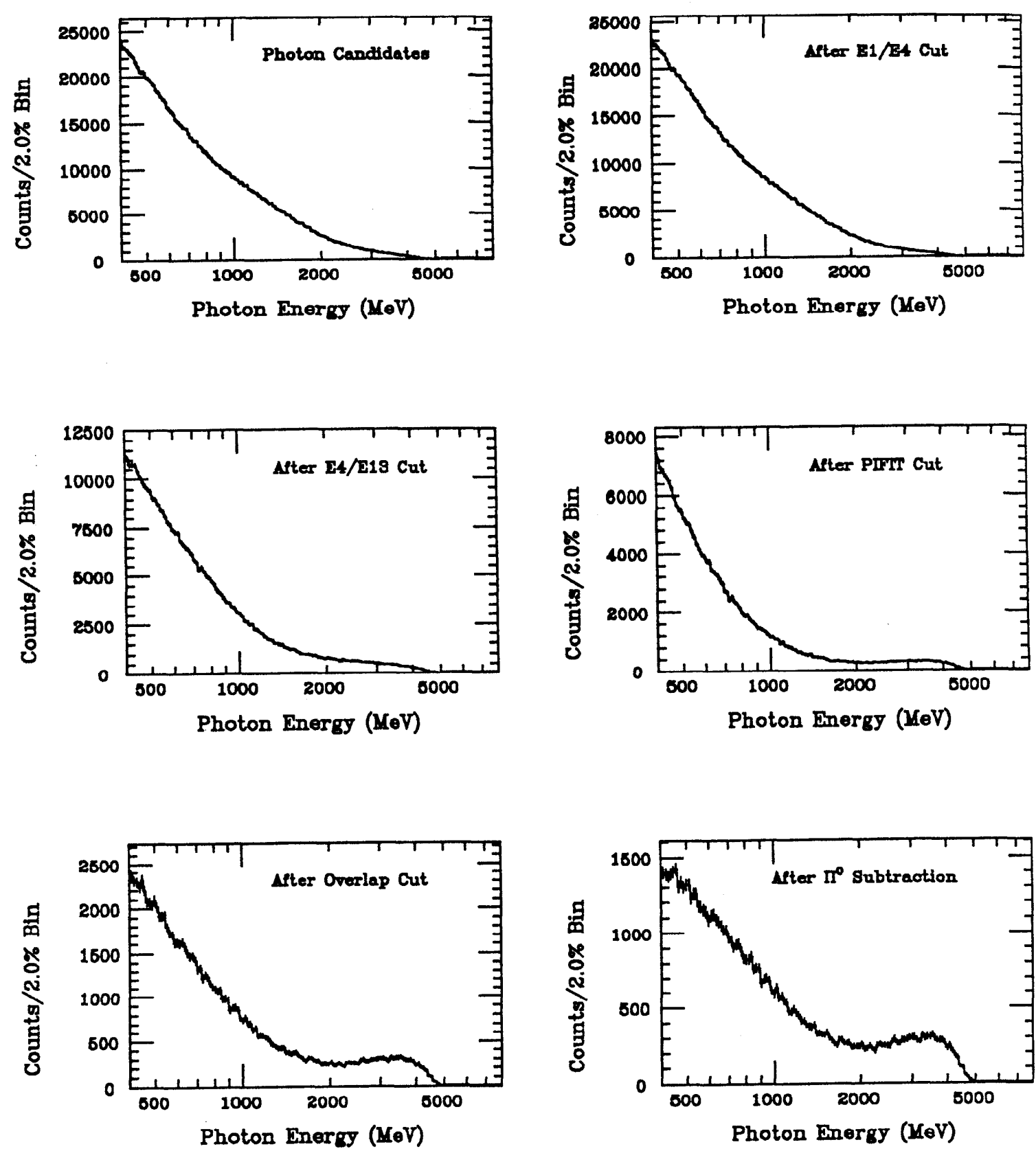

Fig. 4.14 The inclusive photon spectrum after each cut in the analysis. The cuts are taken in the same order as shown in Table 4.4. The final spectrum, labeled "After $\pi^{0}$ Subtraction" is the final inclusive photon spectrum. 


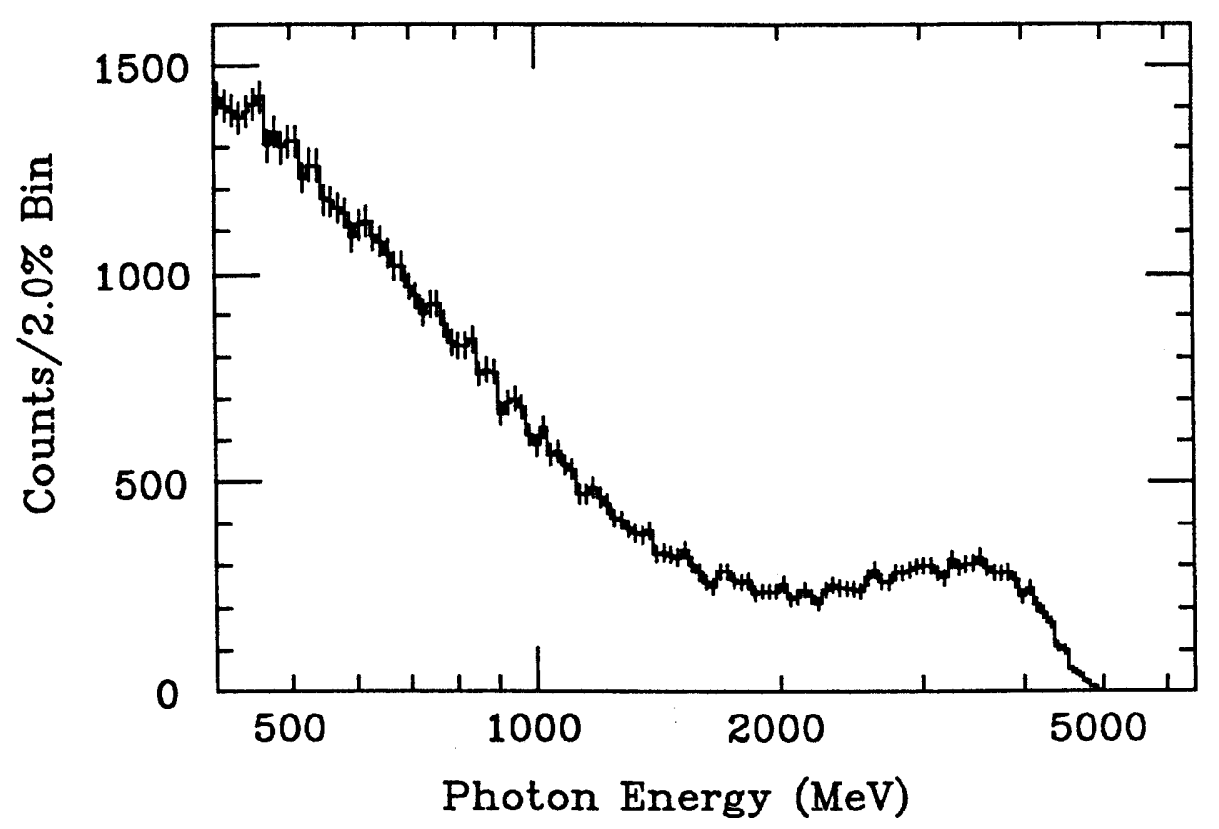

Fig. 4.15 The final $\Upsilon(1 \mathrm{~S})$ inclusive photon spectrum. No obvious narrow structures consistent with the decay $\Upsilon \rightarrow \gamma X$ are indicated.

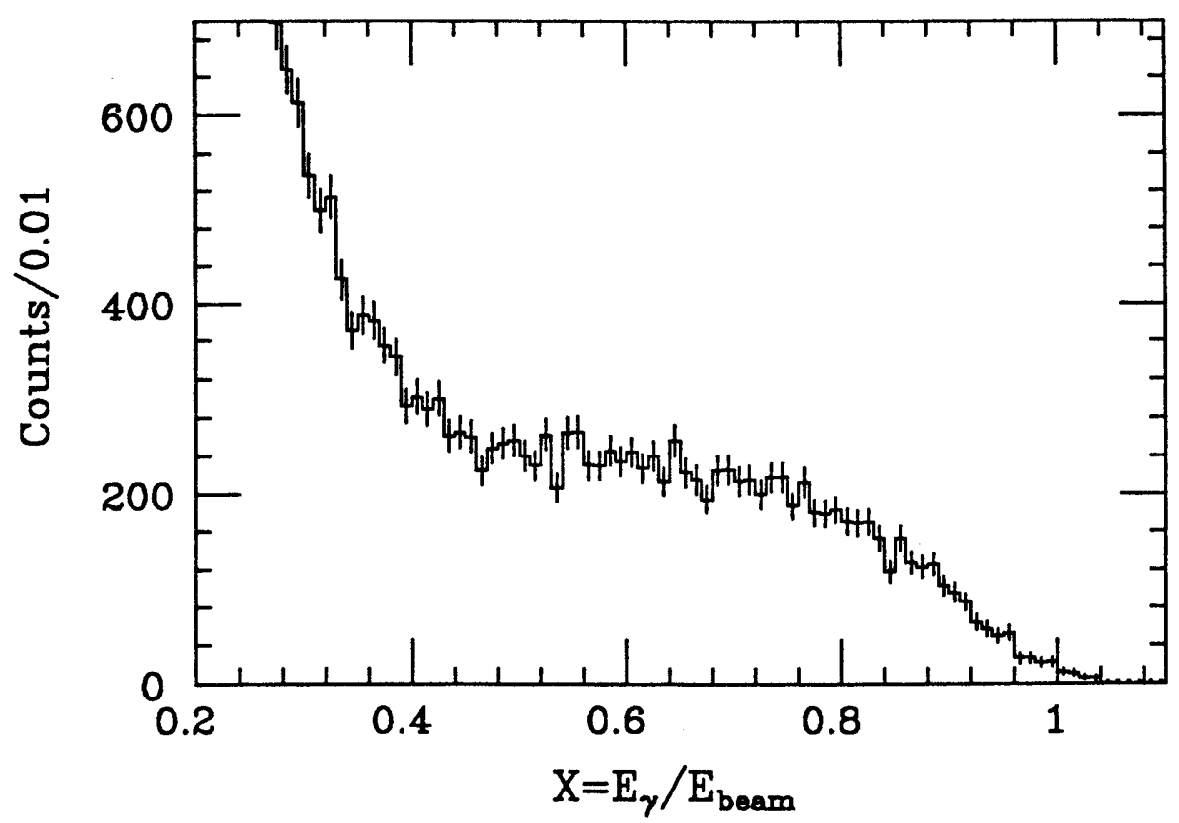

Fig. 4.16 The final $\Upsilon(1 \mathrm{~S})$ inclusive photon spectrum plotted as a function of $x=E_{\gamma} / E_{\text {beam }}$. The broad enhancement from $x=0.5$ to $x=1.0$ is due in part to the decay $\Upsilon(1 S) \rightarrow \gamma g g$. 


\subsection{Photon Efficiency Calculation}

An essential element needed to extract upper limits for the process $\Upsilon(1 \mathrm{~S}) \rightarrow$ $\gamma X$ from Figure 4.15 is the photon efficiency as a function of energy. Because the efficiency for this process depends on properties of the state $X$, some assumptions about its decay modes must be made. In this analysis, $X$ is assumed to decay to all possible fermion-antifermion pairs energetically accessible, where the coupling between $X$ and the $f \bar{f}$ pairs is assumed to be proportional to the fermion mass. These assumptions are those expected for a minimal Higgs particle.

The Monte Carlo events modeling the process $\Upsilon(1 \mathrm{~S}) \rightarrow \gamma c \bar{c}$ which were used to determine the cut values described above are also used to determine the photon efficiency for this process. Because the cuts are optimized on this Monte Carlo data set, systematic errors in the modeling of these decays will result in an overestimate of the efficiency. As discussed above, this effect is reduced by ensuring the cut is a monotonic function of energy and by ignoring points which would make the cut much tighter in a localized energy range (see Figure 4.6 and 4.7, for example). Because the optimization procedure causes such cuts to become tighter, ignoring them in the straight-line fit for an overall, energy-dependent cut, moves the actual cut used in the analysis out to a more conservative value. By examining the distribution for each cut variable at each photon energy and comparing with the actual energy-dependent cut value used, the over-estimate of the efficiency is determined to be not more than about $10 \%$ of the efficiency. Therefore, the final efficiency values are reduced by $10 \%$ to eliminate this bias.

For photon energies above about $4.00 \mathrm{GeV}$, the decay $\Upsilon(1 \mathrm{~S}) \rightarrow \gamma X \rightarrow \gamma c \bar{c}$ is not energetically possible. In this case, the decay $X \rightarrow \tau \bar{\tau}$ is the dominant decay mode. Similarly, above photon energies of about $4.06 \mathrm{GeV}$, the $\tau$ decay mode become inaccessible and the dominant decay mode of $X$ is into strange quark pairs, $X \rightarrow s \bar{s}$. Finally, for photon energies above about $4.68 \mathrm{GeV}$, the dominant decay modes of $X$ are into light quark pairs and $\mu$ pairs. The photon efficiency as a function of photon energy for each of these decay modes is calculated from 
Monte Carlo simulations. The $10 \%$ reduction in efficiency is also applied for each of these processes. The final photon efficiency takes into account the $c \bar{c}, \tau \bar{\tau}$ and $s \bar{s}$ threshold effects and is given by

$$
\epsilon\left(E_{\gamma}\right)=\frac{\sum_{i} \epsilon\left(E_{\gamma}\right)_{f \bar{f}}^{i} C_{f}^{i}\left[M_{f}^{i}\right]^{2}}{\sum_{j} C_{f}^{j}\left[M_{f}^{j}\right]^{2}}\left(\frac{L_{1983}+L_{1984} \times \epsilon\left(E_{\gamma}\right)_{C I S}+L_{1986}}{L_{1983}+L_{1984}+L_{1986}}\right)
$$

where the sums run over the energetically possible fermionic decay modes of $X$, $\epsilon\left(E_{\gamma}\right)_{f \bar{f}}^{i}$ is the efficiency as a function of photon energy of the $i^{\text {th }}$ decay mode, $M_{f}^{i}$ is the mass of the $i^{\text {th }}$ fermion, $C_{f}^{i}$ is the color factor of the $i^{\text {th }}$ fermion, $\epsilon\left(E_{\gamma}\right)_{C I S}$ is the efficiency correction for the 1984 electronics non-linearity described in Appendix A, and $L_{1983}$ is the luminosity obtained in 1983 , and similarly for other years. In other words, the efficiency is the average of efficiencies of the possible decay modes, weighted by the mass squared and the color factor of the decay fermion, and corrected by the electronics induced inefficiency in the 1984 data.

The error on $\epsilon$ is dominated by systematic errors in the Monte Carlo event generation process. The photon pattern cuts depend primarily on the accuracy of the EGS ${ }^{[5]}$ code used to generate electromagnetic showers. As stated in Chapter 3, the EGS code produces shower patterns in close agreement with the data; systematic errors introduced by the loose pattern cuts used in this analysis are therefore small compared to those introduced by other cuts. The HETC ${ }^{|6|}$ code does a reasonable job of simulating the data for the purposes needed here, but is probably the dominant source of systematic errors. The distributions most dependent on HETC code are those dependent on global aspects of the event; the hadron selection routines, the total multiplicity, total energy and particle overlap distributions, and the low energy $\pi^{0}$ multiplicity and momentum distributions. The systematic errors resulting from the hadron selection routines cancel to some extent with those in $\epsilon_{\Upsilon(\mathrm{IS})}^{\text {had }}$ used in section 4.1 to calculate the number of produced $\Upsilon(1 S)$ events. Systematic errors in relation to the total en- 
ergy and event multiplicity distributions have been studied ${ }^{[7,8]}$ and found to be well within $\pm 5 \%$. Only very loose cuts were applied directly to these distributions to define the initial data set, so small differences between the Monte Carlo and data will have a negligible effect for such cuts. The overlap and low-energy $\pi^{0}$ cuts depend indirectly on the event's total multiplicity, and directly on the details of how the Monte Carlo geometrically distributes the energy deposition relative to the photon. The systematic error introduced by these two cuts is estimated to be within $\pm 5 \%$. Finally, the overall systematic error on $\epsilon\left(E_{\gamma}\right)$ is estimated to be $\pm 10 \%$ based on the combination of the above sources of error and on the previous work cited. Note that this systematic error is distinct from the $10 \%$ decrease (which increases the upper limit) already applied to this number.

The Monte Carlo simulations used in the efficiency calculation model the inefficiency caused by photon conversion in the beam pipe or inner chamber layers which results in the photon being detected as a charged particle. The neutral efficiency implicit in the Monte Carlo is about $92 \%$ which is in good agreement with the value of $\mathbf{9 2 . 9 \%}$ from Table $\mathbf{4 . 3}$ for the charge criterion used in this analysis, so no further corrections are applied. Figure 4.17 shows the final photon efficiency as a function of photon energy. The dashed vertical lines indicate the $c \bar{c}, \tau \bar{\tau}$ and $s \bar{s}$ thresholds. The thresholds are assumed to turn on very quickly in this plot; phase-space effects will actually smooth this result.

\subsection{Results}

With the photon efficiency and the number of produced $\Upsilon(1 S)$ in hand, only the number of signal events as a function of photon energy is required to calculate the branching ratio $B R(\Upsilon(1 S) \rightarrow \gamma X)$, with the assumptions on $X$ 's decay modes noted above. The number of signal events is obtained by fitting the inclusive photon spectrum, shown in Figure 4.15, with the detector's response function over a polynomial background. The fit is done in a two-stage process. In the first stage, only a polynomial background, covering about $40 \%$ of the spectrum, 


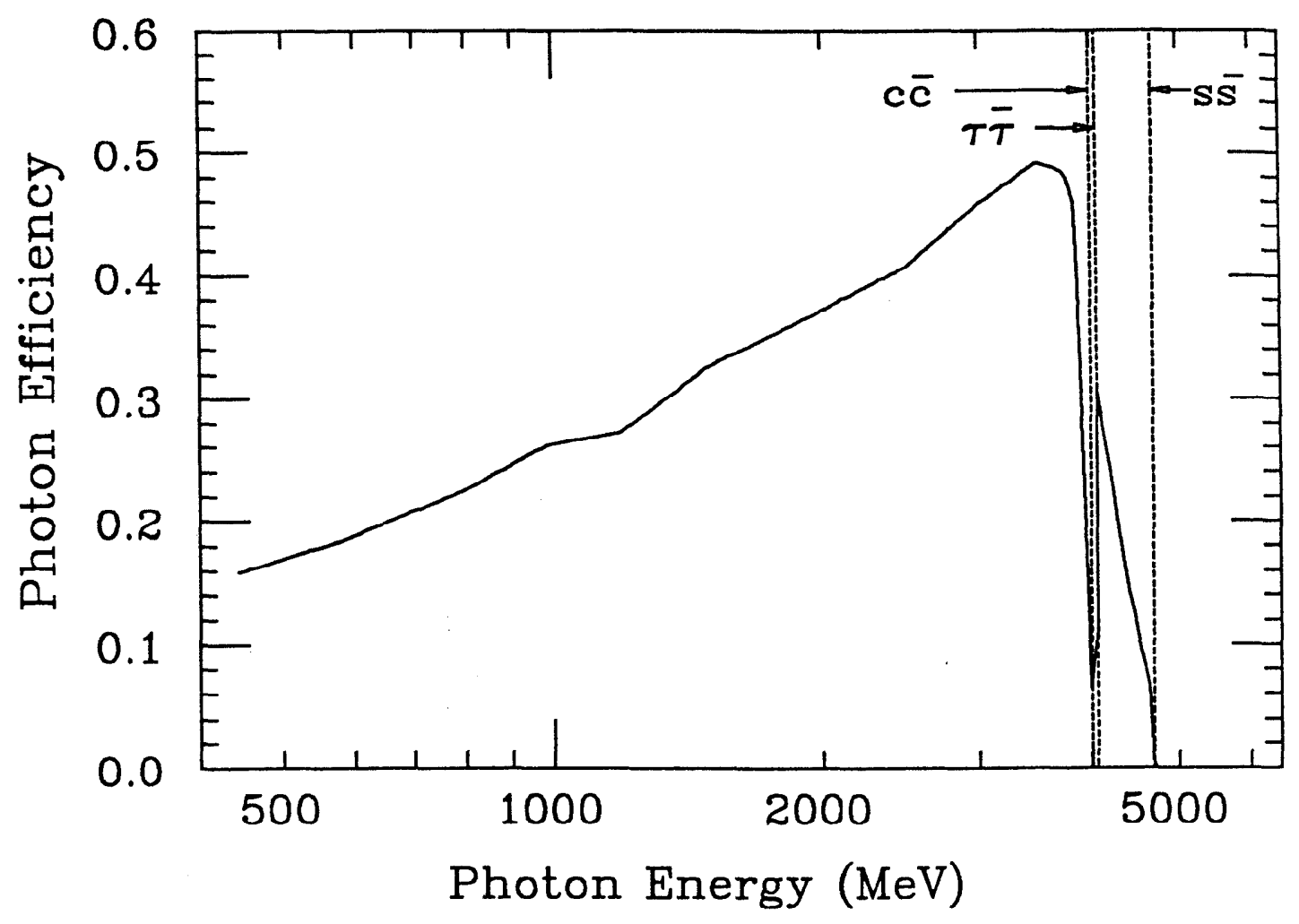

Fig. 4.17 The photon efficiency for the process $\Upsilon(1 \mathrm{~S}) \rightarrow \gamma X$ as a function of photon energy. The state $X$ is assumed to decay into fermion pairs with a coupling proportional to the mass of the fermion. The vertical dashed lines show the kinematic thresholds for the relevant fermions. The thresholds are assumed to turn on very quickly in this plot; phase-space effects will actually smooth this result.

is used in the fit. In the second stage, the detector response function is fixed at a particular energy, with width fixed at $\sigma_{E} / E=2.7 \% / \sqrt[4]{E}$. The background determined from the first stage is added to this response function so that the fit determines only the amplitude of the response function above the fixed background. The energy of the response function is then moved 1\% (each move covers $\frac{1}{2}$ of a $2 \%$ bin) and the fit repeated. This process is repeated over the range that the background is determined from the first stage; approximately $40 \%$ of the spectrum. The background is then successively moved and the above procedure repeated. Each successive background overlaps with the previous one so that 


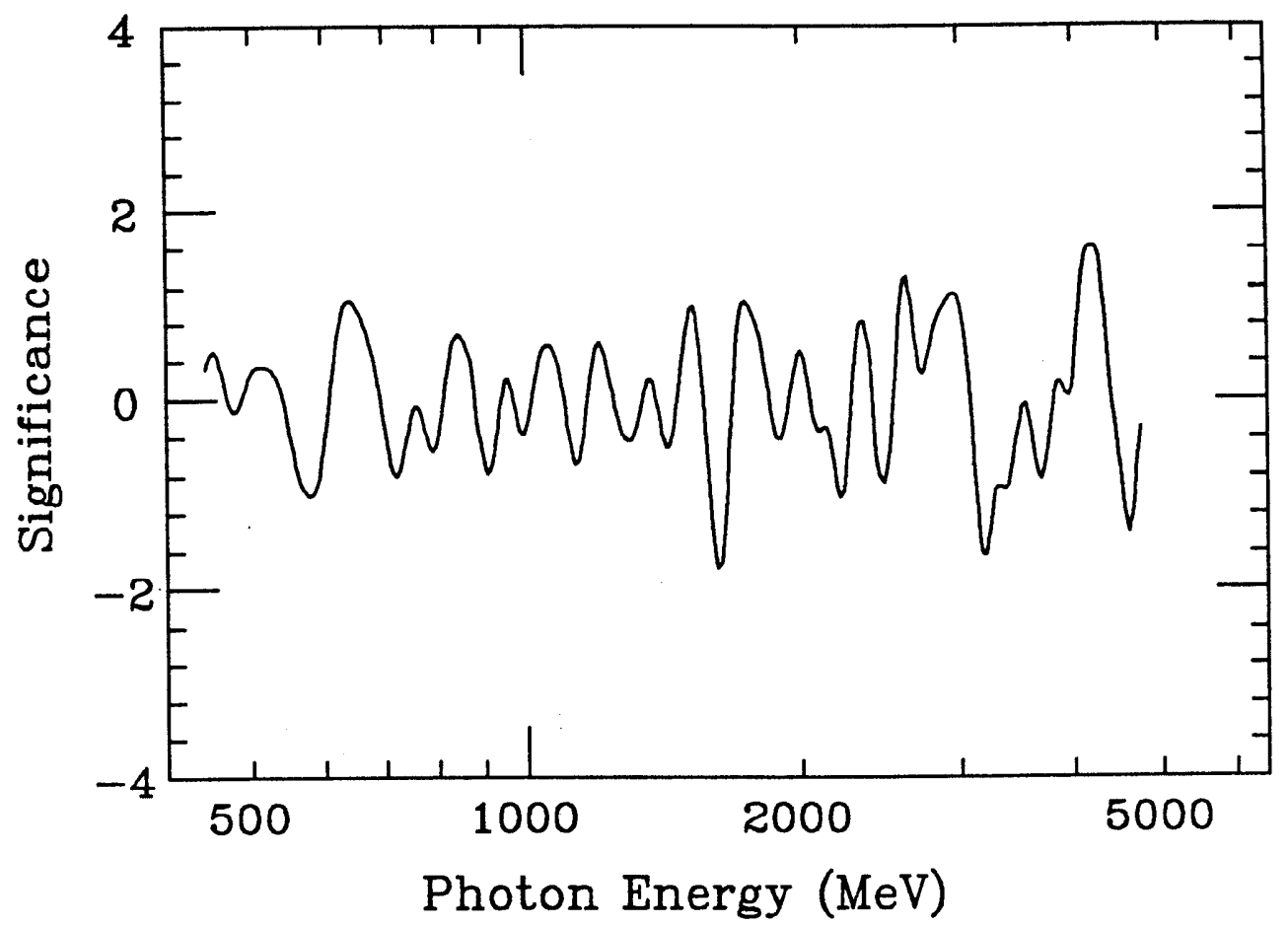

Fig. 4.18 The statistical significance, $S / \Delta S$, of any structure consistent with a narrow state in the inclusive photon spectrum. Negative values correspond to negative fluctuations in Figure 4.15. The most significant structure occurs at $4188 \mathrm{MeV}$ with a significance of less than 1.7.

the only fits used are those whose response function is far from the background end-points.

The result of each fit is an estimate of the number of signal events above background at that photon energy, and a statistical error on that number. To test for any statistically significant structures in the inclusive photon spectrum, the number of standard deviations from zero events as a function of photon energy is shown in Figure 4.18. Each point in this plot is calculated by taking the number of events from the fit and dividing by the error on that number. Negative values in this plot correspond to downward fluctuations in the photon spectrum. The most significant structure in the spectrum lies at $4188 \mathrm{MeV}$ with a significance of less than $\mathbf{1 . 7}$. 
Because no significant structures, consistent with the decay of a narrow state, are seen in the inclusive photon spectrum, upper limits for such states are calculated. The branching fraction is calculated using

$$
f \pm \Delta f=\frac{S \pm \Delta S}{(N \pm \Delta N)(\epsilon \pm \Delta \epsilon)}
$$

where $f$ is the branching fraction, $S$ in the number of signal events from the fit, $\mathrm{N}$ is the number produced $\Upsilon(1 \mathrm{~S})$ events, given by equation [4.3], and $\epsilon$ is the photon efficiency.

In the calculation of upper limits on $f, f$ is assumed to be distributed normally. To convert $f$ and $\Delta f$ to a $90 \%$ confidence level upper limit, a gaussian with mean equal to $f$ and sigma equal to $\Delta f$ is integrated from zero to infinity to get the normalization. The upper limit $U$ is found such that the integral of the same gaussian from zero to $U$ is $90 \%$ of the normalized integral. Thus, $U$ satisfies

$$
\int_{0}^{U} N(x \mid f, \Delta f) d x=0.90 \int_{0}^{\infty} N(x \mid f, \Delta f) d x .
$$

The restriction that the integrals not go negative is due to the physical requirement that branching ratios are always positive. The resulting upper limit curve is shown in Figure 4.19. This result can also be plotted as a function of the recoil mass rather than the photon energy as shown in Figure 4.20. Again, phase-space effects will smooth the thresholds shown in these two plots.

\subsection{Conclusions}

Figure 4.20 indicates that only for Higgs mass around $5.5 \mathrm{GeV} / \mathrm{c}^{2}$ does this analysis come close to the theoretical estimate for the branching ratio of a minimal Higgs particle. For a Higgs mass below about $4 \mathrm{GeV} / \mathrm{c}^{2}$, the efficiency drop, due to Bhabha rejection in the hadron selection routines, causes a large increase in the corresponding upper limit. A different Bhabha rejection algorithm, tuned 


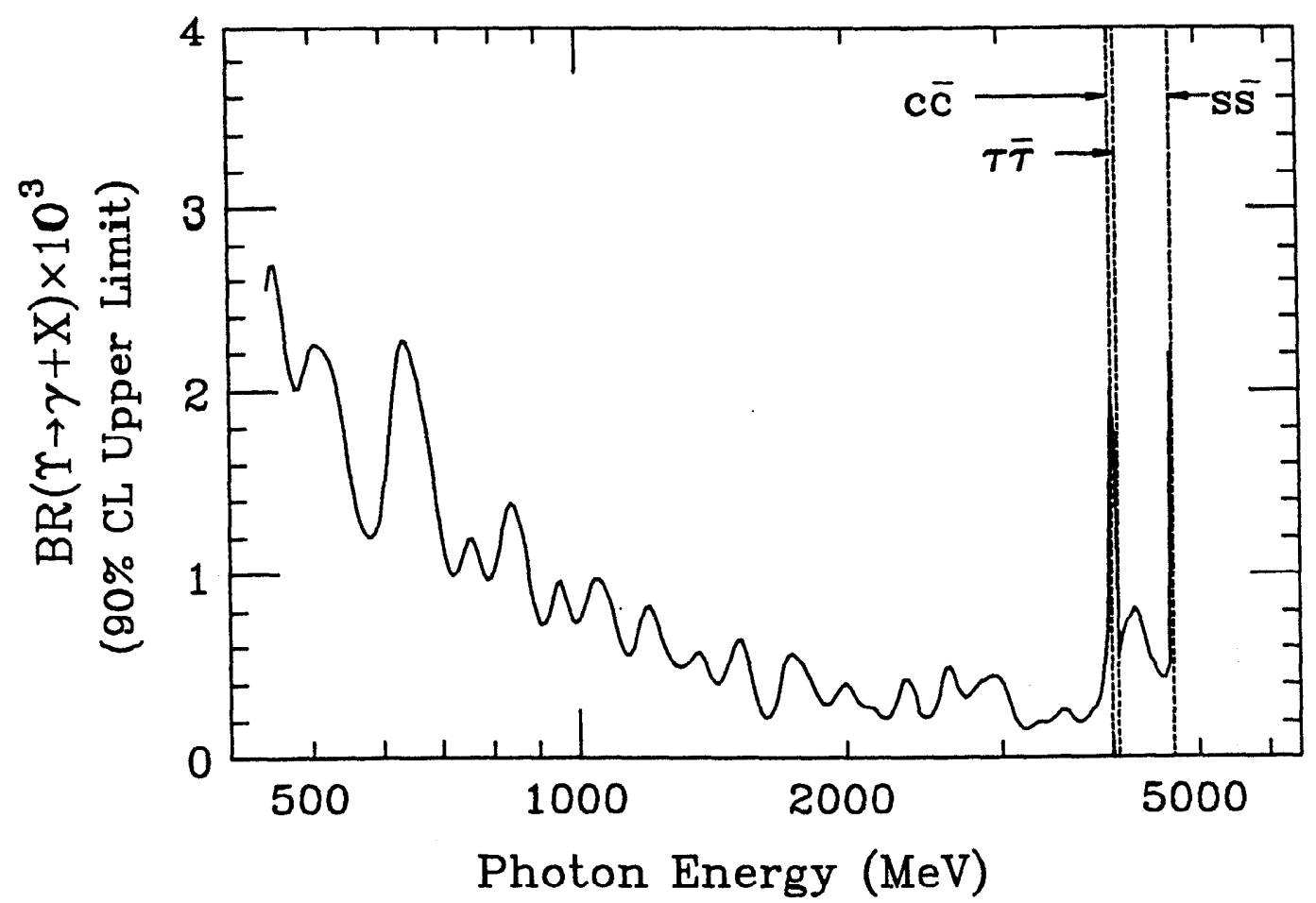

Fig. 4.19 The 90\% confidence level upper limit for the process $\Upsilon(1 \mathrm{~S}) \rightarrow$ $\gamma X$ as a function of photon energy. The assumptions on the decay of $X$ are found in the text. The vertical dashed lines show the kinematic thresholds for the relevant fermions.

for this analysis, might improve the upper limit for Higgs' masses in the 1 to 4 $\mathrm{GeV} / \mathrm{c}^{2}$ range. The decay modes open to the Higgs for masses below about 1 $\mathrm{GeV} / \mathrm{c}^{2}$ are sufficiently different from the decays into $c \bar{c}$ and $s \bar{s}$ that an entirely different analysis would be required to separate such decays from QED events. For a Higgs mass above about $6 \mathrm{GeV} / \mathrm{c}^{2}$, both a slow decrease in photon efficiency and an increase in the number of background photons causes a rise in the upper limit. In no mass range does this analysis rule out a minimal Higgs to the $90 \%$ confidence limit for the latest estimates of the $\Upsilon(1 \mathrm{~S})$ branching ratio which includes QCD radiative corrections.

The above analysis can be applied to different assumptions on the decay modes of $X$. The major difference is that the photon efficiency may have different kinematic thresholds depending on the couplings and the possible decay products 


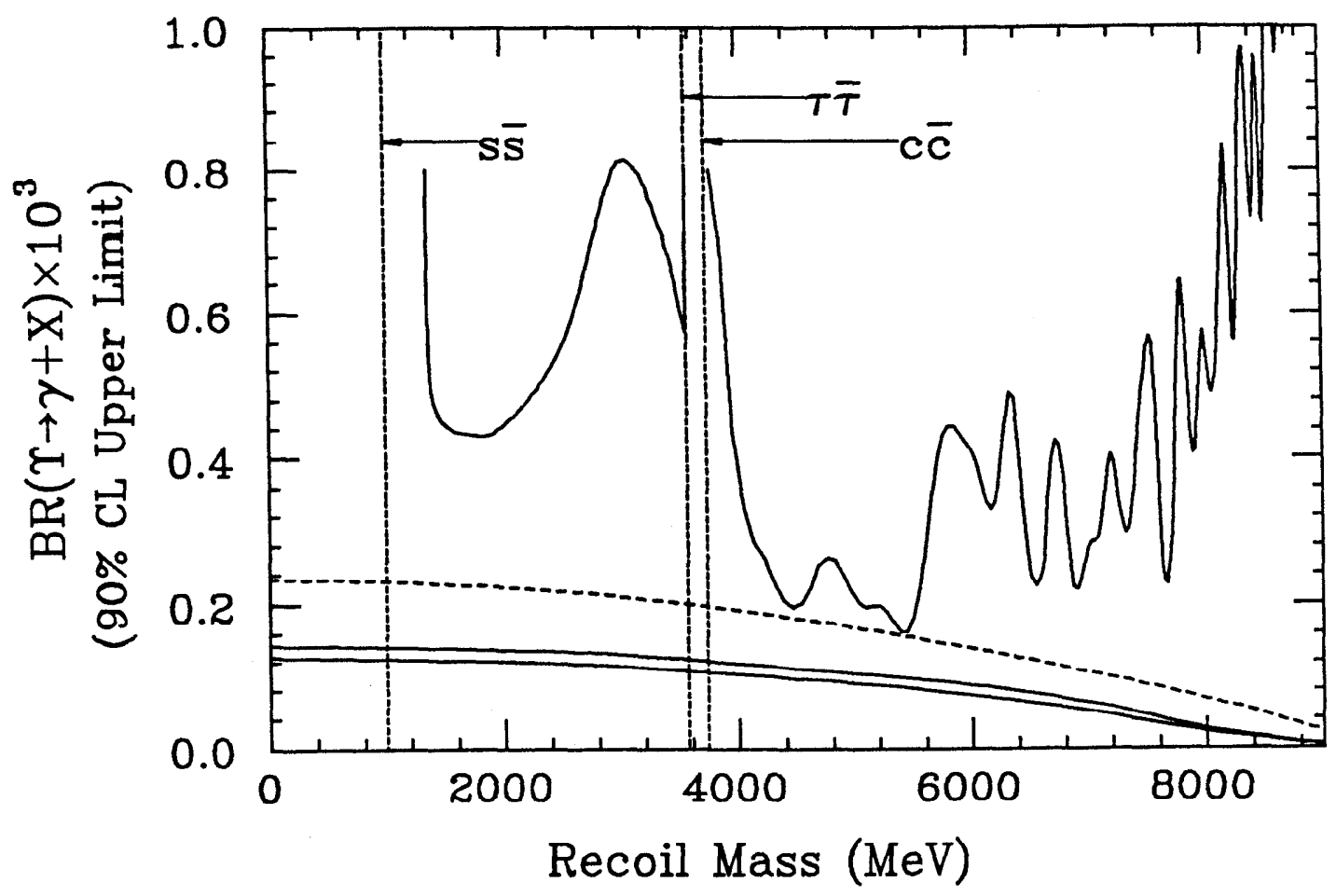

Fig. 4.20 The $90 \%$ confidence level upper limit for the process $\Upsilon(1 \mathrm{~S}) \rightarrow$ $\gamma X$ as a function of recoil mass. The vertical dashed lines show the kinematic thresholds for the relevant fermions. The horizontal dashed line corresponds to the lowest order calculation for the Wilczek mechanism. The two solid horizontal lines indicate the range of the theoretical estimate of the first order radiative corrections to the Wilczek calculation.

involved. If $X$ decays predominantly through low multiplicity exclusive channels, for example, the $\tau \bar{\tau}$ final state predicted to dominate the Higgs decay in some non-minimal models, this analysis will have a lower sensitivity for their detection. The small band between the $\tau \bar{\tau}$ and $c \bar{c}$ thresholds in Figure 4.19 shows this directly. On the other hand, as long as $X$ decays into muti-hadron final states, the detection efficiency remains high, and the upper limits found here will be approximately unchanged.

As stated in Chapter 1, the most promising place to discover a light Higgs particle appears to be in radiative $\Upsilon$ decays. However, a sample of approximately 10 million $\Upsilon(1 S)$ decays recorded by a detector having about 5 times the sensi- 
tivity of the Crystal Ball is needed to reach the current theoretical estimates for masses above $8 \mathrm{GeV} / \mathrm{c}^{2}$. Thus, it will be difficult to substantially improve this result in the near future without new techniques in production and detection of $\Upsilon(1 S)$ events. 
1) Numerical constants taken from Particle Data Group, Rev. Mod. Phys. $\underline{56}$ (1984) S31.

2) As an example of this phonomina, see the analysis by J. Gaiser, Ph.D. Thesis, "Charmonium Spectroscopy from Radiative Decays of the $J / \psi$ and $\psi^{\prime}$, , Stanford University, 1982, SLAC Report 255 (unpublished).

3) R. D. Schamberger et al., Phys. Lett. 138B (1984) 225.

4) S. E. Csorna et al., Phys. Rev. Lett. $\underline{56}$ (1986) 1222.

5) R. Ford and W. Nelson, SLAC report number SLAC-0210, 1978.

6) T. Gabriel et al., Oak Ridge National Laboratory Report, ORNL/TM7123, January, 1981.

7) A more complete treatment of Crystal Ball Monte Carlo simulations is given in Reference 2 above.

8) W. J. Metzger, "Comparisons of Lund Monte Carlo with Crystal Ball Data On and Near the Upsilon", Nijmegen internal report HEN-278. 


\section{Problems in the Crystal Energy Distributions}

\section{A.1 The Symptom}

About nine months after our $1984 \Upsilon(1 S)$ data set was taken, several crystal channels in this data and in data taken in 1985 were found to have an abnormal behavior in their distribution of energy deposition. Figure A.1 shows examples of three such channels, where the problem ranges from minor to severe. About $15 \%$ of the crystals had visible effects in such plots; approximately $5 \%$ were affected in a manner typified by each distribution in Figure A.1. These plots were indicative of a non-linear response to energy deposition. Although $85 \%$ of the crystals showed no indication of a problem, it was difficult to estimate the photon efficiency caused by this, up to then, unknown electronics problem.

Because no problems were seen below about $350 \mathrm{MeV}$, photons with energy below this were assumed unaffected. This was substantiated by noting that, in 1984, the widths and amplitudes for the $\pi^{0}$ and $\eta$ in two-photon invariant mass plots and the $f^{0}$ peak in two-photon annihilations were consistent with those in 1983. For photons of higher energy, several energy dependent effects made efficiency estimation difficult. As the photon energy increases, electromagnetic showers tend to deposit a larger fraction of their energy in neighboring crystals. This fraction is also highly dependent on what part of the crystal the photon enters; photons entering at a corner or edge will deposit less energy in the central 

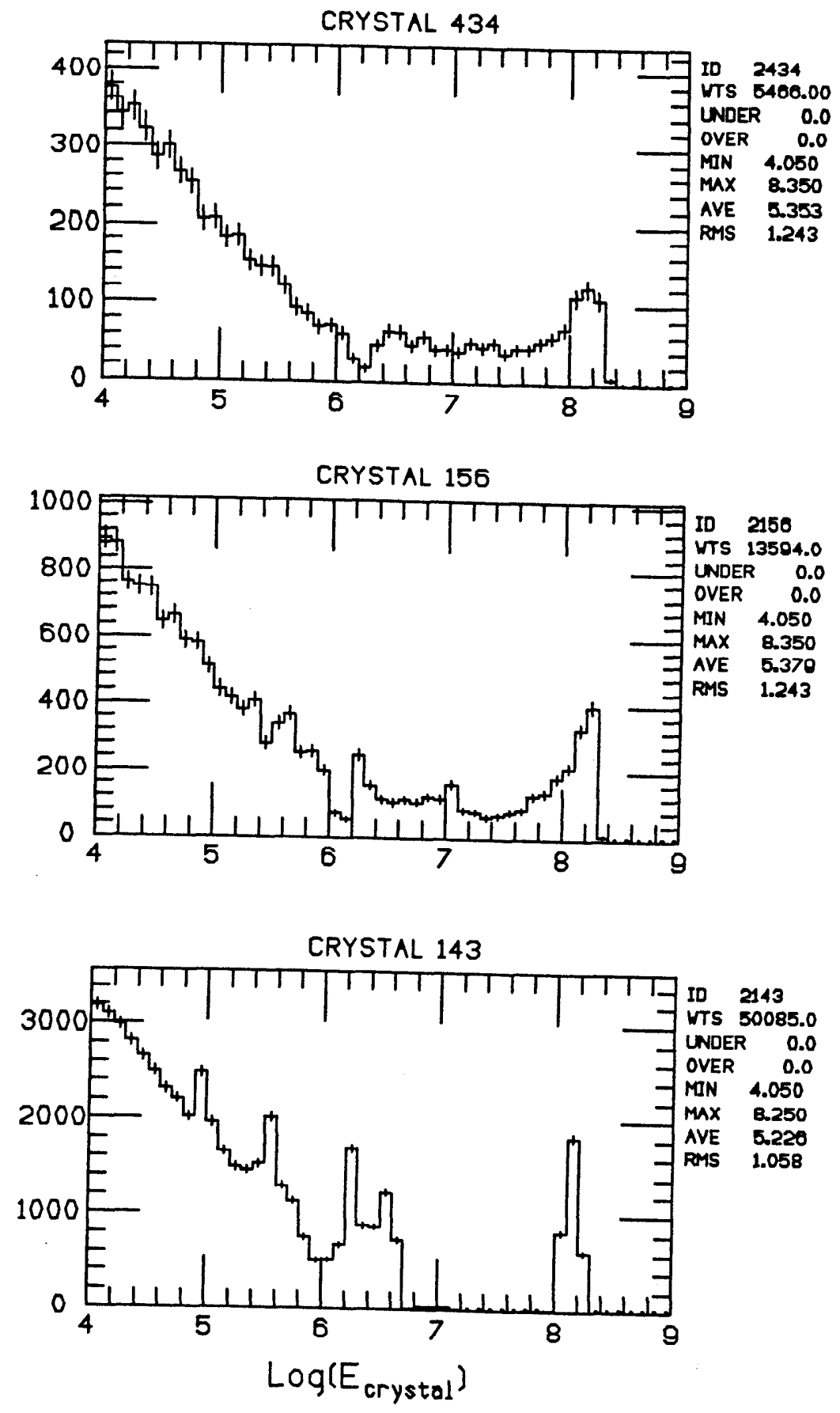

Fig. A.1 Crystal energy distributions in the 1984 data. The energy deposited in the indicated crystal over a long running period is shown. About $5 \%$ of the crystals were affected in a manner typical of each of these 3 plots. 
crystal and more in the neighboring crystals than a photon entering a crystal's center. Because most of the non-linear effects are seen between $400-1000 \mathrm{MeV}$, photons with energies approximately in this range and somewhat higher are assumed suspect. However, because photons with energies between 1000-4700 $\mathrm{MeV}$ can leave large fractions of their energy in neighboring crystals, specifically energy in the 400-1000 MeV range, all photons with energy above about 400 $\mathrm{MeV}$ are suspect.

Although precise efficiency calculations could not be made without a working model of the hardware problem, several observations indicated that the efficiency was not excessively degraded, i.e. below about 70\%. As stated in Section 2.10, Bhabhas are used to calibrate the entire energy range of the Crystal Ball. The 4.7 GeV Bhabhas used for calibrations have roughly $70 \%$ of their energy in the central crystal. This implies that 1 or 2 neighboring crystals could have energy in the 400-1000 $\mathrm{MeV}$ range. If a large number of such Bhabhas were affected by the electronics problem, the energy calibration would have been distorted, resulting in shifted and/or broadened $\pi^{0}$ and $\eta$ peaks. As stated earlier, no such effects were seen. Finally, electronics tests made with the Crystal Ball flasher system indicated the efficiency was low, but these tests were not trusted because they could not predict the actual crystal energy distributions in either quality or quantity. The discrepancy was most likely due to systematic problems with the flasher system itself.

To get a more direct look at the problem, the two-photon decay of $\eta$ 's, where one photon was above $800 \mathrm{MeV}$, was studied. The amplitude and width of the $\eta$ peak in the 1984 data were consistent with the 1983 data, though limited statistics and a difficult background shape made these results somewhat ambiguous. Radiative QED events were also examined. The two primary electrons or photons were used to calculate the expected energy of the smaller, radiative photon. The difference between the expected and calculated energy as a function of the smaller photon's energy in 1984 showed a broadening for about $10 \%$ of the events. 


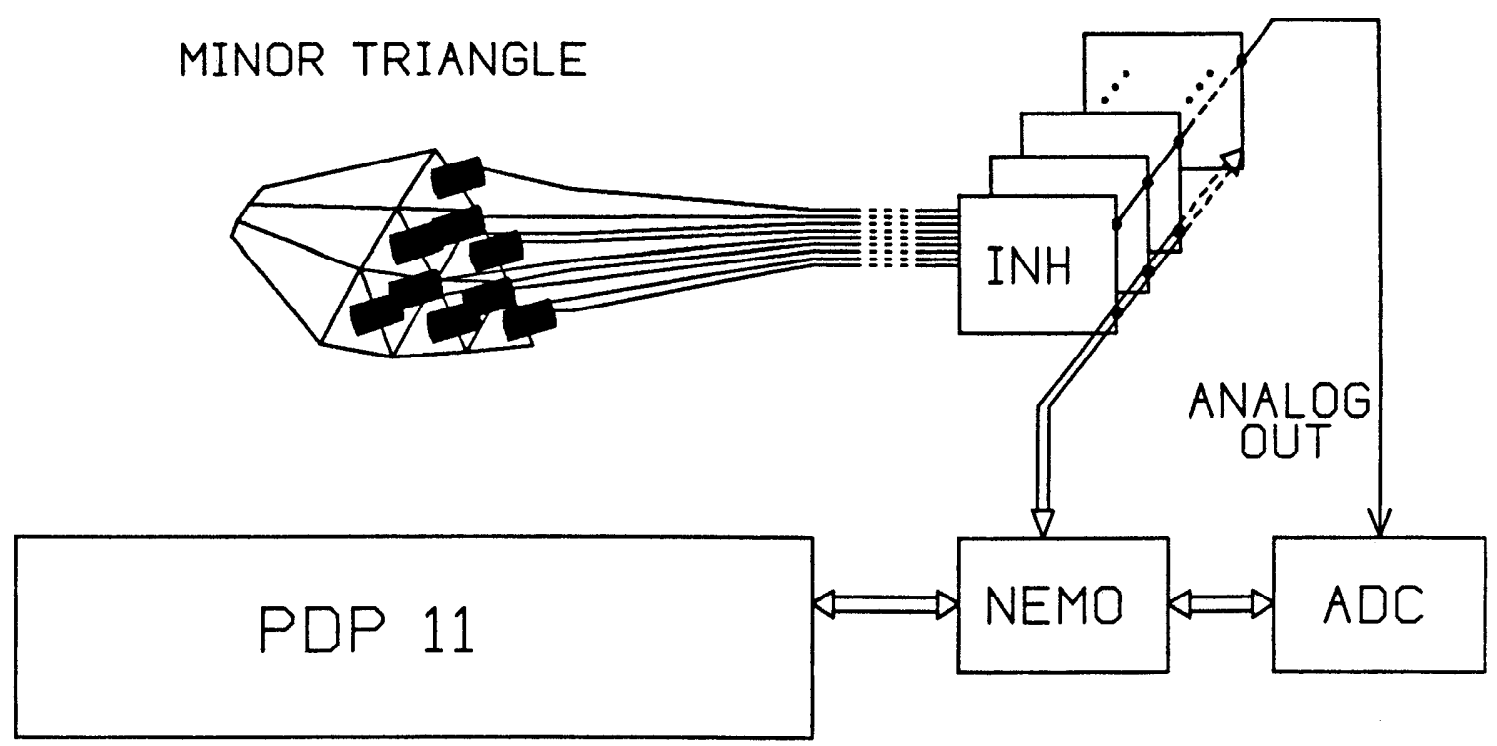

Fig. A.2 The basic setup for the Crystal Ball on-line system. The PDP directs NEMO to select each INH module in turn, where the pulse-height information for each minor triangle is converted by the ADC and sent back to the PDP.

Although these tests were not quantitative, they indicated the efficiency was greater than about $70 \%$. The naive guess of $85 \%$, based only on the number of affected crystal channels, was too rough an estimate and did not give an energy dependence. Without a working model of the non-linearity, and with no effects in the data other than the crystal energy distributions, the photon efficiency as a function of energy was very uncertain.

\section{A.2 The Problem Found}

After examining the on-line electronics, the hardware problem responsible for the non-linear response was discovered. Figure A.2 shows the general features of the Crystal Ball on-line data acquisition system. During normal data taking the 9 phototube outputs for each minor triangle are sent from the detector to the Crystal Ball control room and are processed by "9-Channel Integrate and Hold" (INH) units. The INH divides each signal into a "high channel" and "low 
channel" and amplifies them. The low channel's gain is about 20 times higher than that of the high channel. The pulse's charge is collected on a capacitor for each of the 18 channels. When the experiment is triggered the voltage on each capacitor is held by a FET switch. The on-line PDP then directs "NEMO" to read the voltage on each of these capacitors. NEMO selects an INH unit and has the 18 channels multiplexed, one at a time, to a common output called the Analog Out. It is important to note that NEMO reads the low channel followed by the high channel for each of the 9 crystal inputs. After each channel is selected and allowed to settle NEMO directs the ADC to read the voltage level and transfer the digital information to the PDP. The time delay between NEMO's switch to the next channel and the start of the ADC conversion is called the "NEMO settling time" and is hard-wired in the NEMO module by a component header. After NEMO finishes reading the 18 channels it switches to the next INH and repeats this procedure for all crystal channels.

The first plot in Figure A.3 shows the voltage level of the analog out line as NEMO switches through the 18 channels of an INH module. A pulser was used to simulate NaI signals into the 9 input channels of the INH. The high voltage levels correspond to the "low channels" while the lower voltage levels correspond to the "high channels" because the low channels have a higher gain. As the input pulse height increases (B), the voltage levels rise until the low channel outputs saturate at around 11 Volts (C). As the input pulse height increases further (D), the high channel continues rising while the low channel voltages drop somewhat. Because the ADC times out for signals above 8 Volts, and because the off-line software uses the high channel information in this case, the low channel behavior is unimportant for higher pulse heights. The width of each plateau increases with increased voltage level because the ADC takes longer to convert a larger signal.

In an effort to reduce the experiment's dead time, the NEMO settling time referred to above was reduced from $10.8 \mu \mathrm{sec}$ to $5.6 \mu \mathrm{sec}$ in March 1984. This drastically reduced the time allowed for the switching transients to stabilize be- 

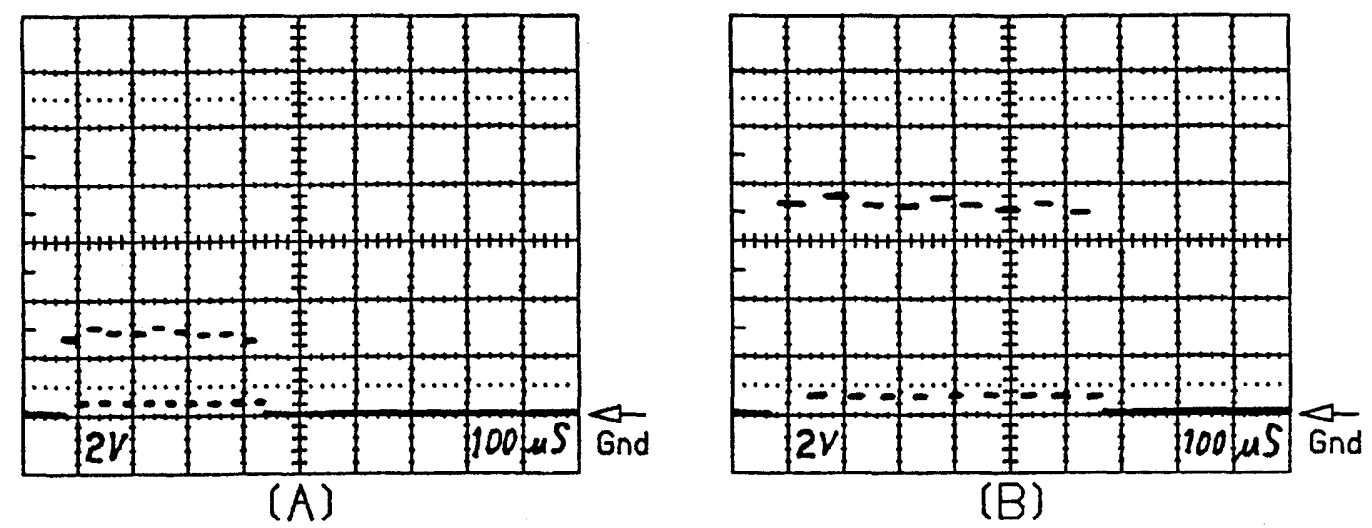

(C)
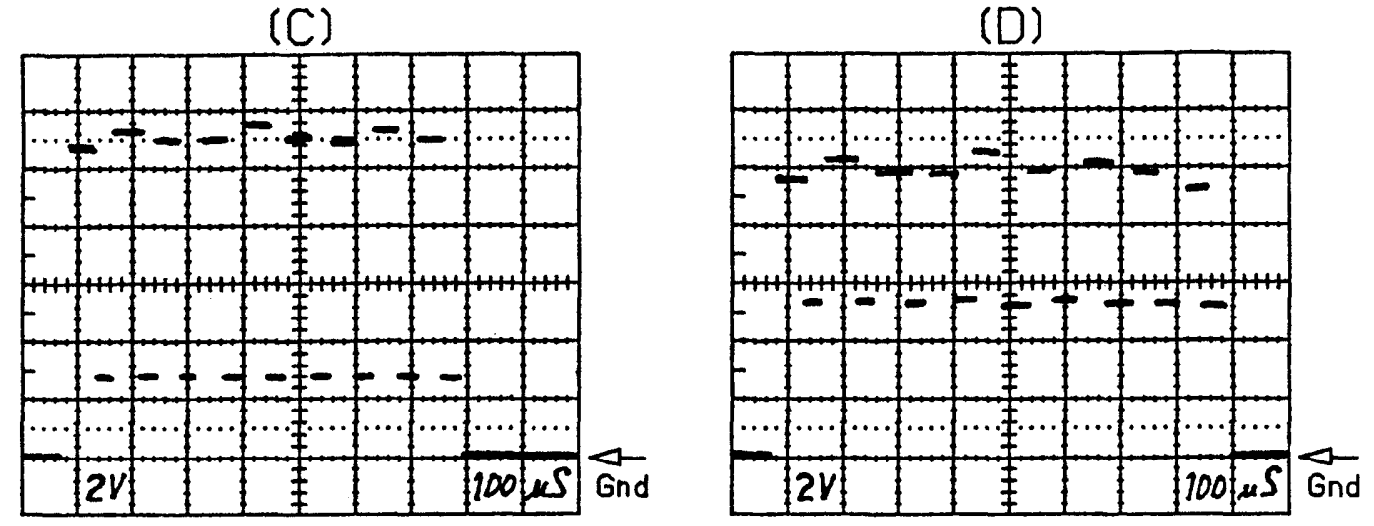

Fig. A.3 The voltage level for the Analog-Out scan. The nine input channels are selected by NEMO, low channel first followed by the high channel. The plateaus with the higher voltage levels are the low channels. The figures show a progression of increased input pulse-height. Note the low channel saturates at about 11 volts. The vertical voltage scale is 2 Volts/division and the horizontal time scale is $100 \mu \mathrm{sec} /$ division. 
tween channel changes. To reduce this required transition time, a "pull-down" FET switch was added to ground the Analog Out line just after each ADC read. The duration of this grounding was only a small fraction of the total settling time. The addition of the pull-down FET greatly decreased the settling time from the low to high channel transition because the low-channel voltage level is higher than the high channel's voltage. At first this modification might seem naive; on transitions from the high to the following low channel, there is a voltage increase which is impeded by the grounding FET. The reason this modification works is due to the output op-amp, described below, being able to drive the Analog Out line up in voltage much faster than down. For most crystal channels the change to the $5.6 \mu \mathrm{sec}$ settling time with the pull-down FET installed, reduced a safety factor but did not affect the electronic's performance. In a small fraction of channels, however, this delay was too short and the ADC conversion began before ringing had subsided. This effect was also found to be pulse-height dependent and explains why only energies in an intermediate energy range were affected, as explained below. It should be pointed out here that in retrospect, this change was not the only one to cause an electronics failure. Interference between this modification and another electronics change, made a few months after the $1984 \Upsilon(1 S)$ run, exacerbated the problem. The other change will be discussed below.

Figure A.4 shows a close-up of an improperly working channel in the AnalogOut scan (the grounding FET is out of the circuit in these plots for clarity). The voltage level falls from the low-channel plateau toward the high-channel level, rings and then settles to the correct value (A). Also shown is the start-ADC pulse. This pulse gates the Analog-Out line to create an input pulse for the ADC. As the input pulse-height increases (B), the voltage difference between the low and high channels steadily grows. This requires more time for settling to the high-channel voltage level. If the input pulse-height increases further, the lowchannel voltage level remains saturated at around 11 volts (and even decreases 


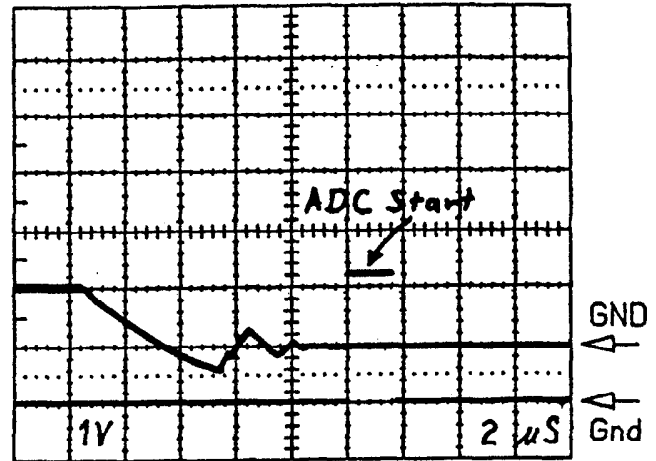

(A)

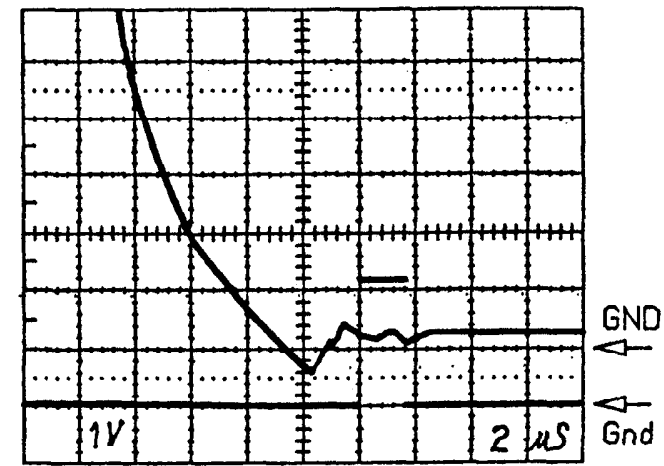

(B)
(C)

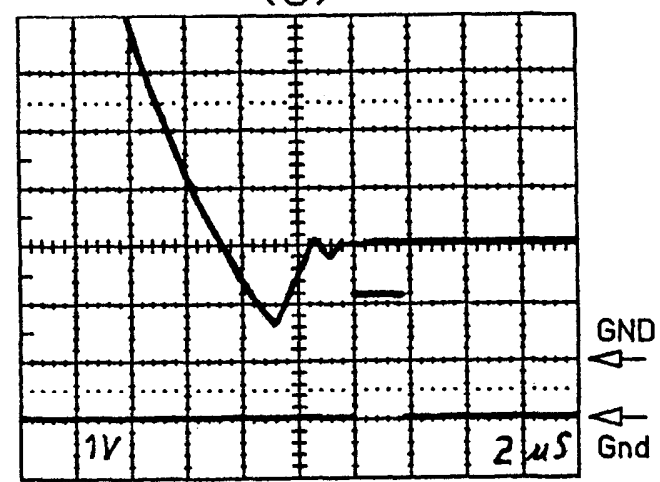

(D)

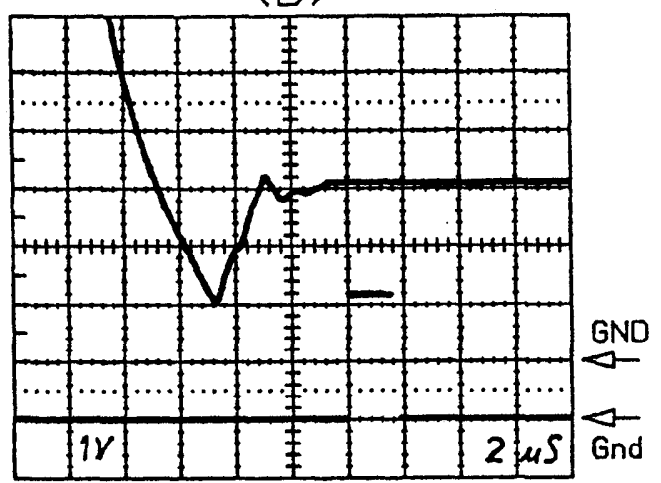

Fig. A.f One Channel in the Analog-Out scan. As the pulse-height input increases, the time required to reach a stable high-channel level increases, until the low-channel saturates. This is the point where the voltage difference between the low and high-channel levels is greatest and incorrect conversion values are measured (B). Further increase in input pulse-height only decreases the voltage difference and thus the time for settling. The vertical voltage scale is 1 Volt/division and the horizontal time scale is $2 \mu \mathrm{sec} /$ division. The time of ADC conversion is the 2.2 Volt pulse starting at $12 \mu \mathrm{sec}$. Ground for the analog out line and the ADC convert pulse is labeled GND and Gnd respectively. 


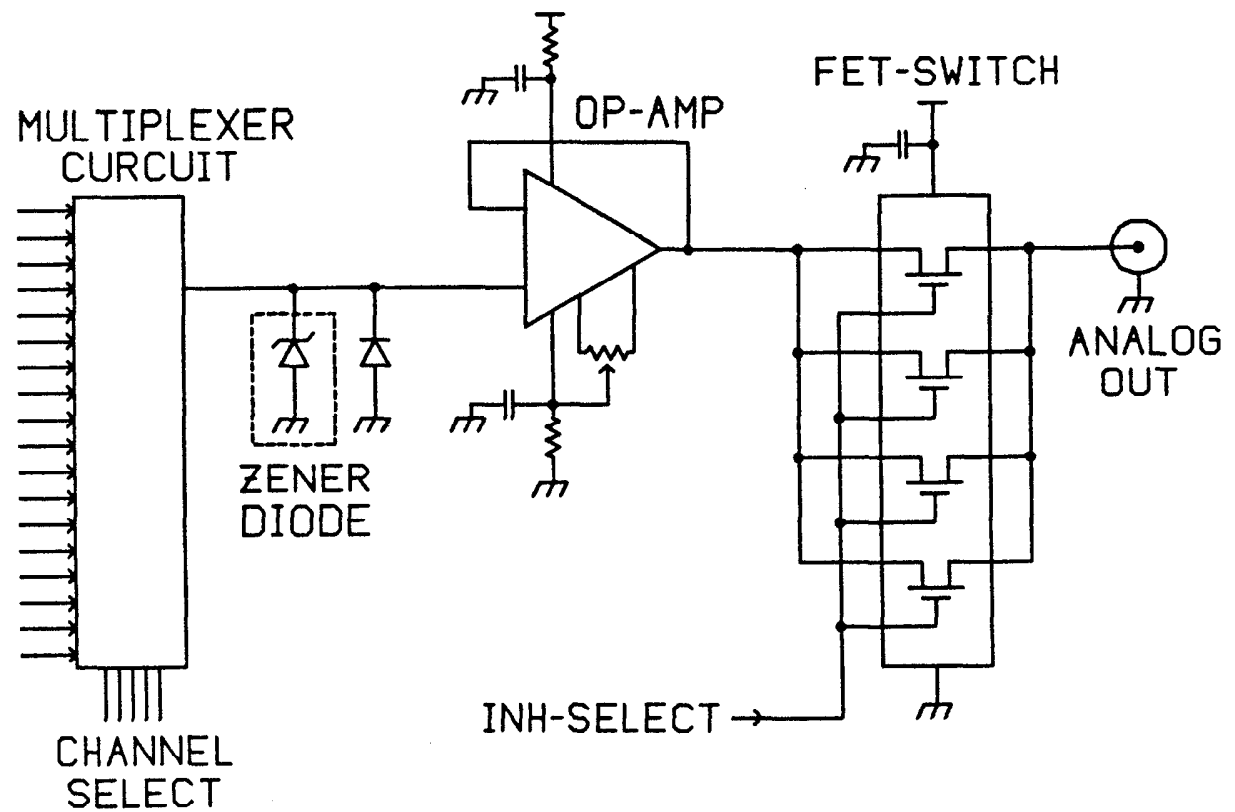

Fig. A.5 The output schematic for the INH output circuit. The CA3140T op-amp determines the rise-time between channel switches while the CD4066A FET switch determines the ringing just before reaching the low-channel plateau.

somewhat) while the high-channel voltage level rises. This makes the voltage difference decrease (C-D). Thus, the time needed for settling from the low to the high channel increases with input pulse height until the low channel saturates, then slowly decreases. In this example, incorrect voltage values are converted for the cases shown in (B) and (C).

Figure A.5 shows the output circuit for an INH module. The final CA3140T op-amp dictates the fall time from the low to high channel plateaus. Manufacturing variations in these op-amps cause the fall-time to vary by about $1 \mu \mathrm{sec}$. These variations are illustrated in Figure A.6. Resistance and capacitance values for the final FET switch in Figure A.5 are responsible for the ringing seen just before reaching a stable high-channel plateau. Figure A.7 shows the effect of two different FET's in the INH output circuit. The top plot (A) shows the result of using the design (RCA-CA4066A) FET switch. The lower figure (B) shows 

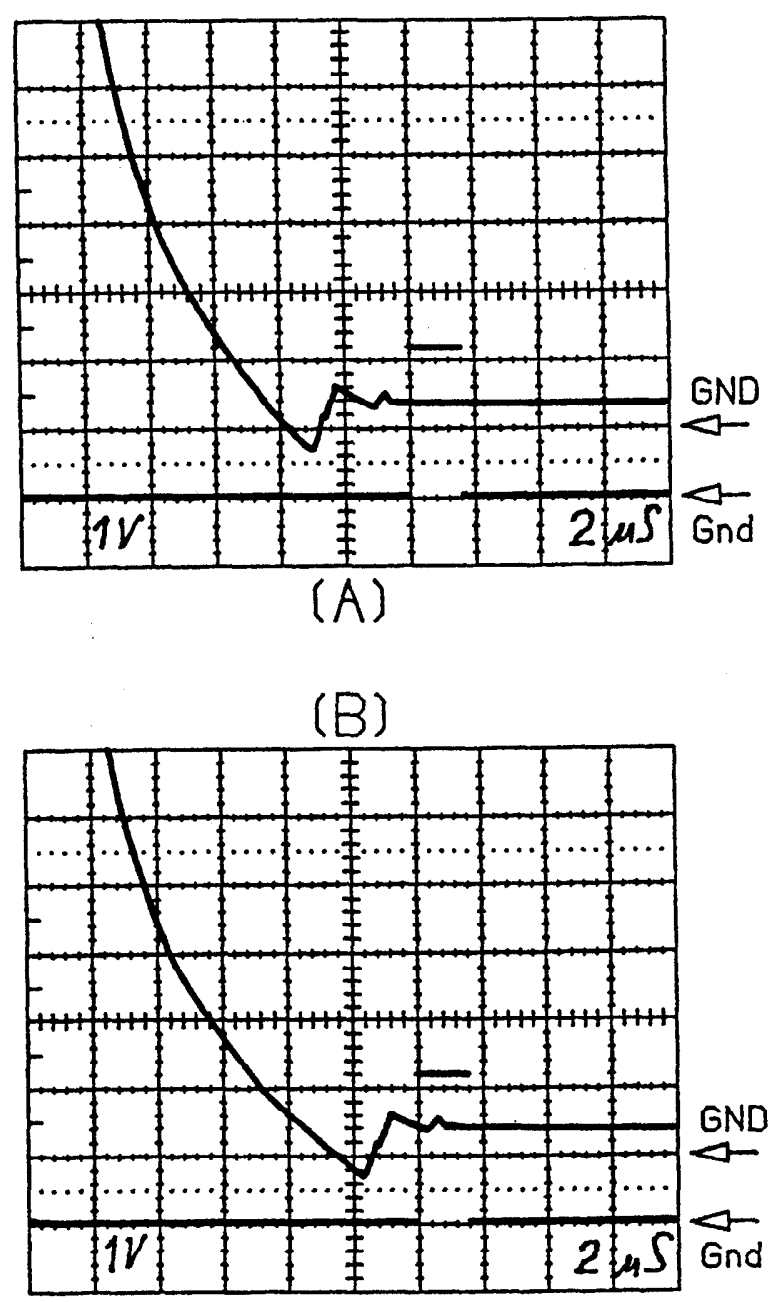

Fig. A.6 The effects of different op-amps on the settling time. The top plot shows a "good" op-amp when a stable high-channel level is reached before the ADC conversion begins. The bottom plot shows a "bad" op-amp. The difference is due to variations in their manufacturing. The vertical voltage scale is $1 \mathrm{Volt} /$ division and the horizontal time scale is $2 \mu \mathrm{sec} /$ division. The time of ADC conversion is the 2.2 Volt pulse starting at $12 \mu \mathrm{sec}$. Ground for the analog out line and the ADC convert pulse is labeled GND and Gnd respectively. 

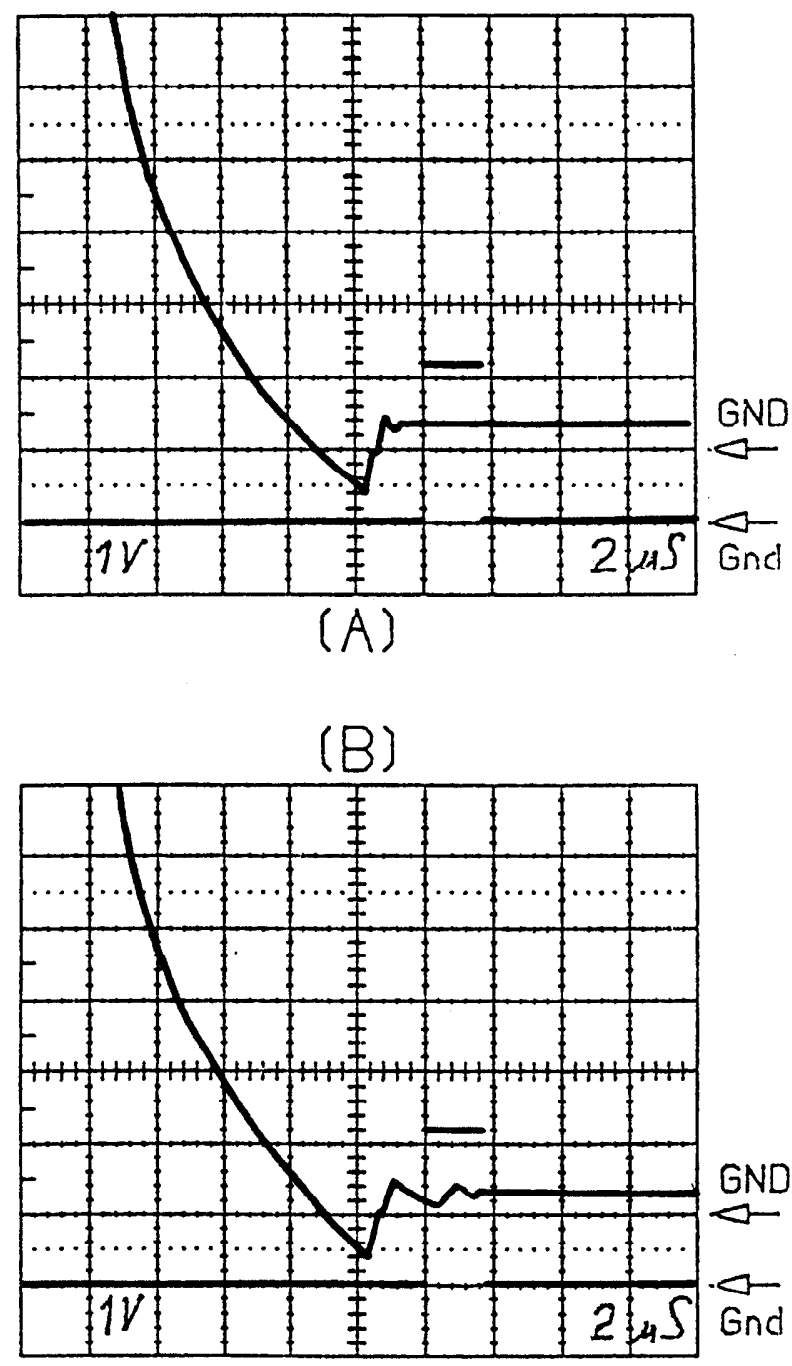

Fig. A.7 The effects of different FET switches on the settling time. The top plot shows little ringing from the use of an RCA FET switch. The bottom plot shows more ringing, resulting in a longer settling time for an HEF FET switch. This difference is due to a difference in component specifications. The vertical voltage scale is 1 Volt/division and the horizontal time scale is $2 \mu \mathrm{sec} /$ division. The time of $A D C$ conversion is the 2.2 Volt pulse starting at $12 \mu \mathrm{sec}$. Ground for the analog out line and the ADC convert pulse is labeled GND and Gnd respectively. 
the effect of what was thought to be a compatible replacement (HEF-CA4066B). The settling behavior is seen to be quite degraded using the HEF switches. This can be traced to different manufacturing specifications on the CA4066A verses CA4066B chips. Thus, the HEF chips require about an additional $2 \mu$ sec for proper settling behavior. In February 1985, all of the INH output FET switches were replaced because it was thought some were leaking when closed. About $25 \%$ were replaced by spare RCA FET chips and the rest by the HEF FET chips. The settling time degradation of the HEF switches was not known at the time. The combination of this change and the lowering of the NEMO settling time reduced any safety factor to essentially zero for all crystal channels. Manufacturing variations in critical components, especially the variation noted in the op-amps, allowed the ADC read to occur before the analog line had stabilized. This explains why most crystal channel problems were in the $400-1000 \mathrm{MeV}$ region; this is precisely the range where the low channel becomes saturated and the necessary settling-time to the high channel is a maximum. This corresponds to photons with energy in the $500-1500 \mathrm{MeV}$ range. This is just in range of the 1 $\mathrm{GeV}$ signal reported as preliminary in the summer of 1984 which prompted the $1984 \Upsilon(1 \mathrm{~S})$ run.

In summary, the $1984 \Upsilon(1 \mathrm{~S})$ data was degraded by lowering the NEMO settling time and installing the pull down FET. This effect occurred only in the high channel and was seen in about $15 \%$ of the crystals. After the fall 1984 running, the RCA FET switches were changed to predominantly HEF FET switches which further degraded the electronics' performance. This change, in combination with some preliminary measurements made with the Crystal Ball flasher system before the actual problems were found, gave erroneous efficiency estimates. Although these measurements did not correctly predict the energy distributions in the 1984 data, they did add confusion to an already uncertain situation. 


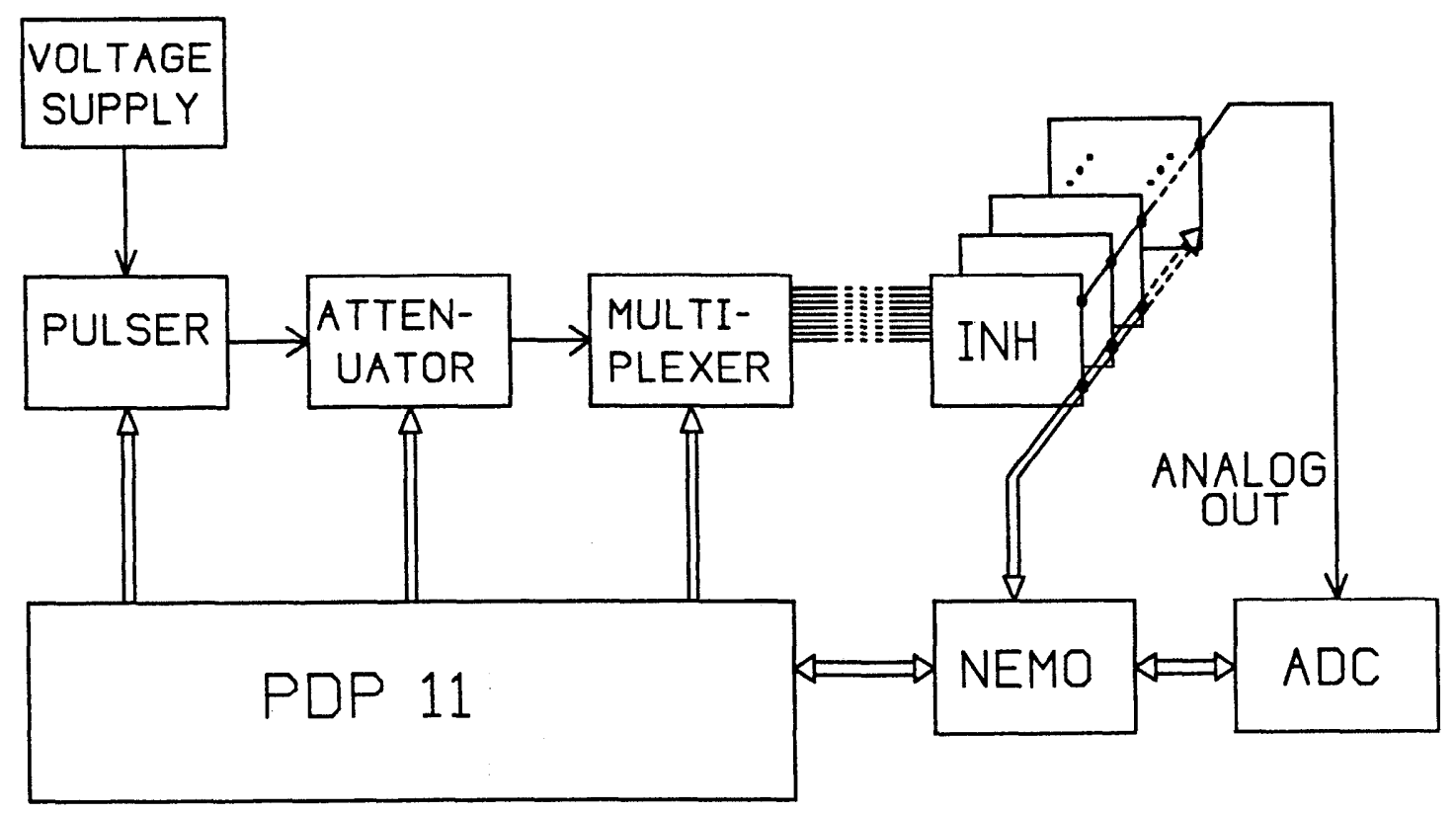

Fig. A.8 The electronics setup for the charge injection system. A NaI pulse is generated and attenuated before entering the selected channel of an INH unit. The rest of the on-line data acquisition system performs as it does during normal data taking.

\section{A.3 Measurement of the Non-linear Response}

Once the electronics' problems responsible for the non-linear response were found, it was clear that measuring the response function for all crystals could result in an accurate determination of the photon efficiency as a function of energy. To measure the electronic's response to energy deposition, a charge injection system (CIS) was constructed and used to simulate NaI pulses entering the INH units ${ }^{\dagger}$ Figure A.8 shows the basic setup. Here the phototube output is simulated with a NaI pulse generator. The pulse's amplitude is defined by the voltage level driving the generator. The pulse is attenuated by any of 5 possible resistor networks which can be inserted into the circuit by the PDP. These 5 attenuator circuits reduce the pulse's amplitude by a factor of $16,4,2,1.4(\sqrt{2})$

† This system was initially used to check the linearity of the electronics when the Crystal Ball was first set-up at DESY in late 1982. No problems were observed at that time. 
and $1.2(\sqrt[4]{2})$ respectively. This provides 32 possible attenuation levels, each level differing from the previous one by about $20 \%$. The attenuated pulse then enters a 16-channel multiplexer box, also controlled by the PDP. The first 9 channels are used to select which INH input channel the pulse will enter.

To measure the response function, the PDP selects a channel and attenuator setting and causes a pulse to be generated. The experiment is triggered and all data from the event is read by the PDP in the usual manner. A program in the PDP then extracts the low and high ADC counts for the pulsed channel and saves them for further analysis. The 5 attenuators are changed to the next setting and the process repeated for all 32 possible levels from the highest attenuation setting to no attention. This procedure is repeated 5 times for the selected channel. The multiplexer box then selects the next channel in the INH which is measured similarly. When all 9 channels have been measured, the cable from the multiplexer box is moved to the next INH unit and the above steps are repeated for all $88 \mathrm{INH}$ units. To increase the measurement's resolution, the voltage level driving the pulse generator is changed and the entire procedure repeated. The voltage change is designed so that the two sets of 32 attenuator settings mesh in an alternating fashion. This was very useful for seeing fast changing response curves and, as discussed below, useful for determining time dependent systematic errors. The process is fully automated except for the cable change to the next INH unit. The entire measurement takes 16-18 hours.

It would have taken much less time to make 5 pulses for each attenuator setting rather than running through the 32 settings 5 times. The attenuator settings were changed with each pulse because the results were dependent on the mechanical relay contacts which take the attenuators in or out of the circuit. If several pulses were taken without changing the relays, the average ADC counts read were very stable with a sigma on the order of $0.01 \%$ for a medium range pulse-height. However, if the relays were changed, and the measurement repeated, the results would again show a very small error but often inconsistent 


\begin{tabular}{|c|c|c|c|}
\hline \multicolumn{4}{|c|}{ Hardware Configurations for CISSY Tests } \\
Run & INH FET & NEMO Settling Time & Pull-Down FET \\
\hline 1983 & All A & $10 \mu \mathrm{sec}$ & No \\
\hline 1984 & A and B & $5 \mu \mathrm{sec}$ & Yes \\
\hline 1985 & A and B & $10 \mu \mathrm{sec}$ & Yes \\
\hline
\end{tabular}

Table A.1 The electronics' hardware configuration for the 1983 and 1984 running periods. The hardware was in the 1985 configuration when the electronics problems were discovered.

with the first measurement. Cleaning the relay contacts helped considerably but they were still the dominant point to point error. In this case, the sigma was about $0.05 \%$. When a measurement using this method was repeated after $1-2$ days a difference of less than about $2 \mathrm{ADC}$ counts over the entire attenuation range was seen. This was assumed to be pedestal drifts and/or temperature effects. Thus, the measurement errors included the statistical error from the 5 pulses and a systematic error of 2 ADC counts. Because data for the two different voltage settings were taken many hours apart, every other point in the 64-point response curve was taken at very different times. This helped to determine the longer term systematic errors by observing systematic shifts between adjacent points.

In late 1985, the measurement described above was performed with the detector in the 1983 and 1984 configurations as defined in Table A.1. Shown in Figure A.5 is a Zener diode which was added between the 1984 and 1985 data taking runs. This modification was made so that after a channel saturated it would stay at 10 volts. This was useful for diagnostics, but it did seem to change the settling behavior slightly. Therefore, the Zener diodes were removed from all INH modules for the 1983 and 1984 simulations. The 1983 set-up was modeled first and involved changing the NEMO settling time to $10.8 \mu \mathrm{sec}$ and removing the 
pull-down FET. All the INH output FET's were changed to the RCA CA4066A type which were in place for the 1983 and 1984 running. Because the actual chips in use in 1983 and 1984 were discarded in February 1985, replacements of the same type and manufacturer were used. For the 1984 measurement the NEMO settling time was set at $5.6 \mu$ sec and the pull-down FET was installed.

\section{A.4 Modeling the Problem}

To model the problem in the 1984 data, the ratio of 1984 to 1983 ADC counts was formed for all crystal channels over the electronics' entire dynamic range. Care was taken to treat the low and high channel data in the same manner as done in the off-line software. The response curve was determined for only 64 discrete values so ADC counts between those measured were modeled by linear interpolation. A software program called CISSY was developed to take an energy assumed to be in the 1983 data, and predict the energy found in the 1984 data, based on the measured response curves. By applying these predicted distortions to the 1983 crystal energy distributions, a prediction for the 1984 distributions is obtained which can be compared to the actual ones. Figures A.9, A.10 and A.11 show such plots for the crystals shown in Figure A.1. The agreement is usually very good, however some crystal channels were good in the $1984 \Upsilon(1 \mathrm{~S})$ data with the predicted plots showing non-linearities, while others were bad in the 1984 data with the predicted plot showing no indication of a problem. At least three effects are presumably responsible for this:

- In the year between the 1984 data taking and the charge injection measurements taken in November 1985, a few INH modules were swapped; no record was kept of this change.

- During this period, some channels had their op-amps and/or FET switches replaced. A record of these changes was kept, but was lost.

- The behavior of some channels may have changed during this year.

To model these effects, a search was made for channels with non-linearities 
CRYSTAL 434
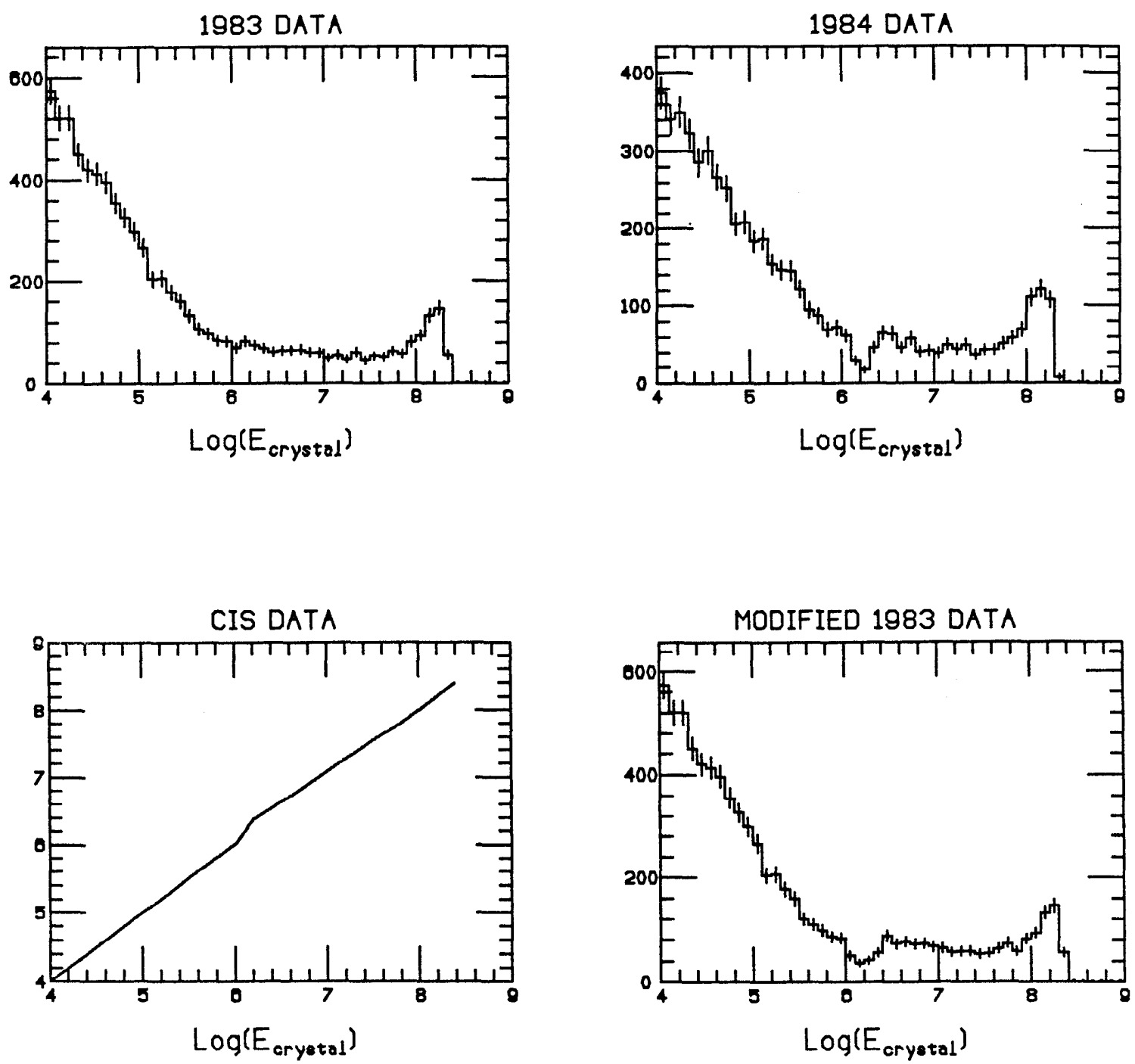

Fig. A.9 Comparison of predicted and measured crystal energy distributions for crystal 434. The plot in the upper left and upper right show the 1983 and 1984 data respectively. The lower left plot shows the distortion function calculated from CISSY. The lower right plot is the convolution of the upper left and lower left plots and is a prediction of the 1984 data. 
CRYSTAL 156
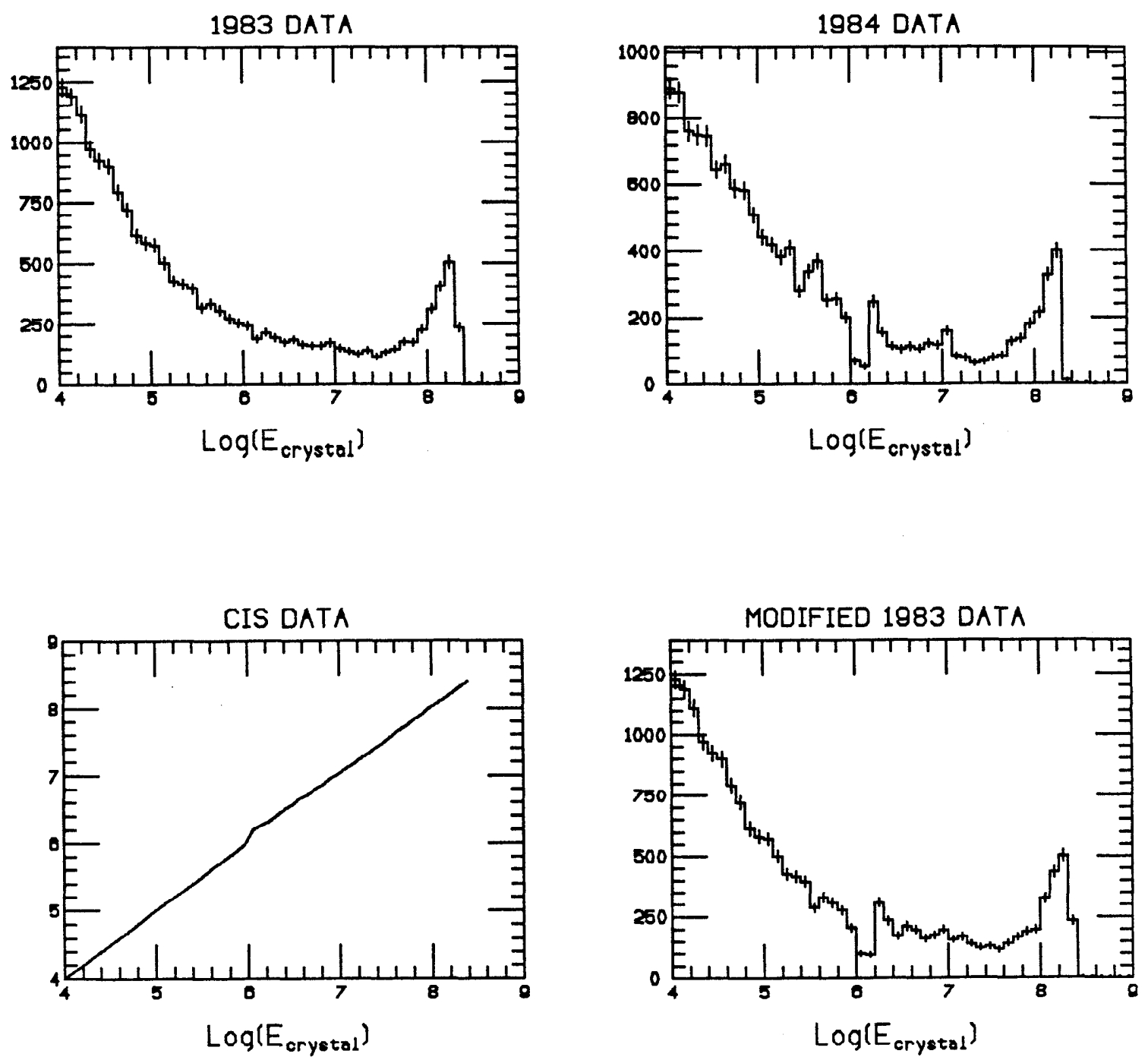

Fig. A.10 Comparison of predicted and measured crystal energy distributions for crystal 156. The figure descriptions are the same as in Figure A.9. 


\section{CRYSTAL 143}

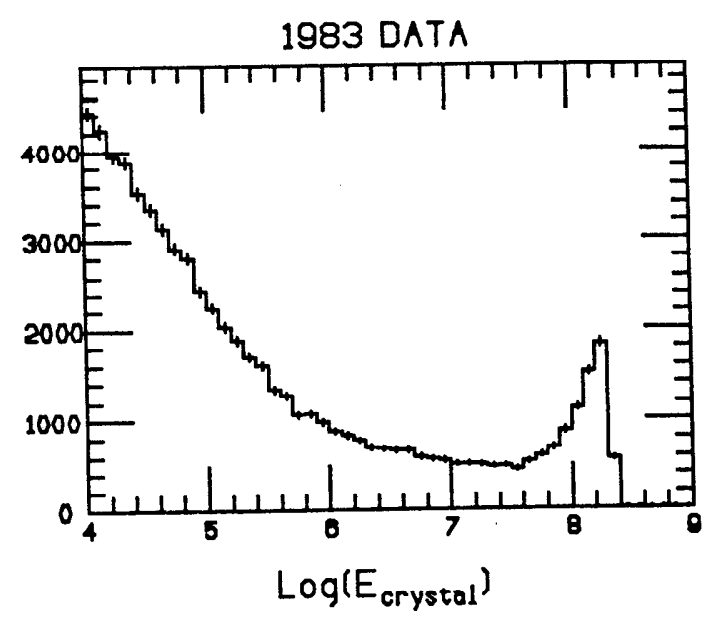

1984 DATA
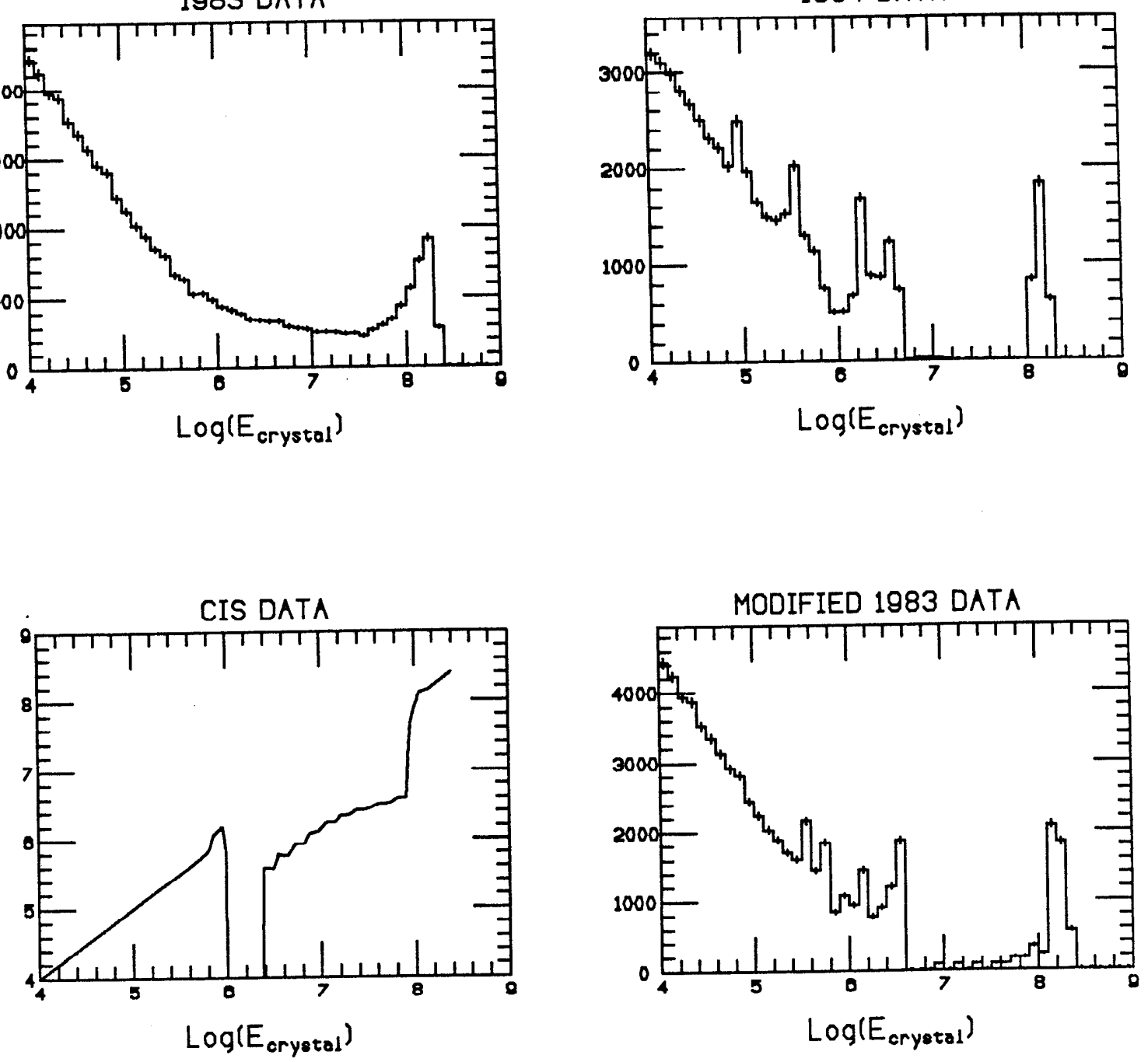

Fig. A.11 Comparison of predicted and measured crystal energy distributions for crystal 143. The figure descriptions are the same as in Figure A.9. Note the saw-toothed pattern in the non-linear region of the response curve. This is due to a systematic change between the two measurements taken with different pulser voltages. Because this behavior is not seen in the linear regions, this indicates the non-linearity is not stable over the several hour period between the two sets of data taking. 
consistent with INH swapping, and the charge-injection data for these channels were interchanged. Seven such swaps were modeled, affecting about $2.5 \%$ of the channels. The remaining channels which showed no problem in the 1984 data but had a non-linearity in the CIS data were identified. Another channel in the same INH, showing no non-linearities was used to model these channels. Approximately $1.0 \%$ of the channels were of this type. Finally, channels which appeared normal in the CIS data but had problems in the 1984 data were modeled with another channel showing a similar non-linearity. About $1.1 \%$ of the channels were of this type.

One final modification was done to the CIS data; those channels which showed a much better or much worse non-linearity than was warranted from the 1984 energy-distributions were modeled with another non-linear channel with a similar behavior. About $2.7 \%$ of the channels fell in this category; about equal number of channels were improved as were degraded. With these modifications, CISSY reliably modeled the problems found in the 1984 crystal energy distributions for all crystal channels.

\section{A.5 Results of CIS Measurements}

The primary interest in CISSY is in obtaining a photon efficiency curve as a function of energy. This is done by generating Monte Carlo $\Upsilon \rightarrow \gamma c \bar{c}$ events for a variety of photon energies and merging them with real events triggered only by the DBM trigger. Merging the DBM events help simulate machine related backgrounds not in the Monte Carlo, as described in section 3.5. The photon spectrum for these events is made and compared to the spectrum after convoluting all energies with CISSY and reanalyzing the events with the standard off-line production software. The ratio of the number of peak photons after running CISSY to the number before is the efficiency. Because every Monte Carlo event has a photon with the energy being tested, the photon spectrum has a very large peak. This represents a $100 \%$ branching ratio of the $\Upsilon$ into $\gamma c \bar{c}$. An 


\begin{tabular}{|c|c|c|}
\hline \multicolumn{3}{|c|}{$\begin{array}{c}\text { Photon Cuts for CISSY Analysis } \\
\text { "Soft" Cuts }\end{array}$} \\
"Medium" Cuts & "Hard" Cuts \\
\hline$\left|\cos \theta_{Z}\right| \leq 0.875$ & $\left|\cos \theta_{Z}\right| \leq 0.850$ & $\left|\cos \theta_{Z}\right| \leq 0.825$ \\
\hline $0.40 \leq E_{1} / E_{4} \leq 0.980$ & $0.50 \leq E_{1} / E_{4} \leq 0.960$ & $0.55 \leq E_{1} / E_{4} \leq 0.955$ \\
\hline $0.70 \leq E_{4} / E_{13} \leq 0.995$ & $0.78 \leq E_{4} / E_{13} \leq 0.985$ & $0.80 \leq E_{4} / E_{13} \leq 0.982$ \\
\hline $\cos \theta_{A B} \leq 0.90$ & $\cos \theta_{A B} \leq 0.85$ & $\cos \theta_{A B} \leq 0.82$ \\
\hline $\operatorname{Pifit} L L D \leq 1.50$ & Pifit LLD $\leq 1.00$ & Pifit LLD $\leq 0.85$ \\
\hline$\left|M_{\pi}-M_{\gamma \gamma}\right| / M_{\pi} \geq 0.15$ & $\left|M_{\pi}-M_{\gamma \gamma}\right| / M_{\pi} \geq 0.15$ & $\left|M_{\pi}-M_{\gamma \gamma}\right| / M_{\pi} \geq 0.15$ \\
\hline
\end{tabular}

Table A.2 The cuts used to define photon in the efficiency calculation.

actual spectrum does not show such large peaks so this approach is not entirely valid. The main systematic error here is in the fitting procedure; the line-shape and background level are not well represented. With a more realistic branching ratio, say around $1 \%$, the fit is insensitive to the line-shape. This can be modeled by using only a small number of Monte Carlo generated events and a statistical background, roughly simulating the level seen in the actual data.

Another method of estimating the photon efficiency is to insert Monte Carlo photons into real data. In this case, however, the higher energy photons are no longer a negligible fraction of the event's total energy. Adding such photons to an otherwise typical event will violate energy and momentum conservation and will neglect angular correlations present in a real event. Thus, this method was not used for an efficiency estimation.

The results of the $\Upsilon \rightarrow \gamma c \bar{c}$ analysis are shown in Figures A.12 and A.13. Two thousand $\Upsilon \rightarrow \gamma c \bar{c}$ events were generated for ten different photon energies ranging from $400 \mathrm{MeV}$ to $3500 \mathrm{MeV}$. Photons from these events are subjected to three sets of cuts; "soft" cuts which have a high efficiency but low background rejection, "medium" cuts which are typical of most Crystal Ball analyses, and 

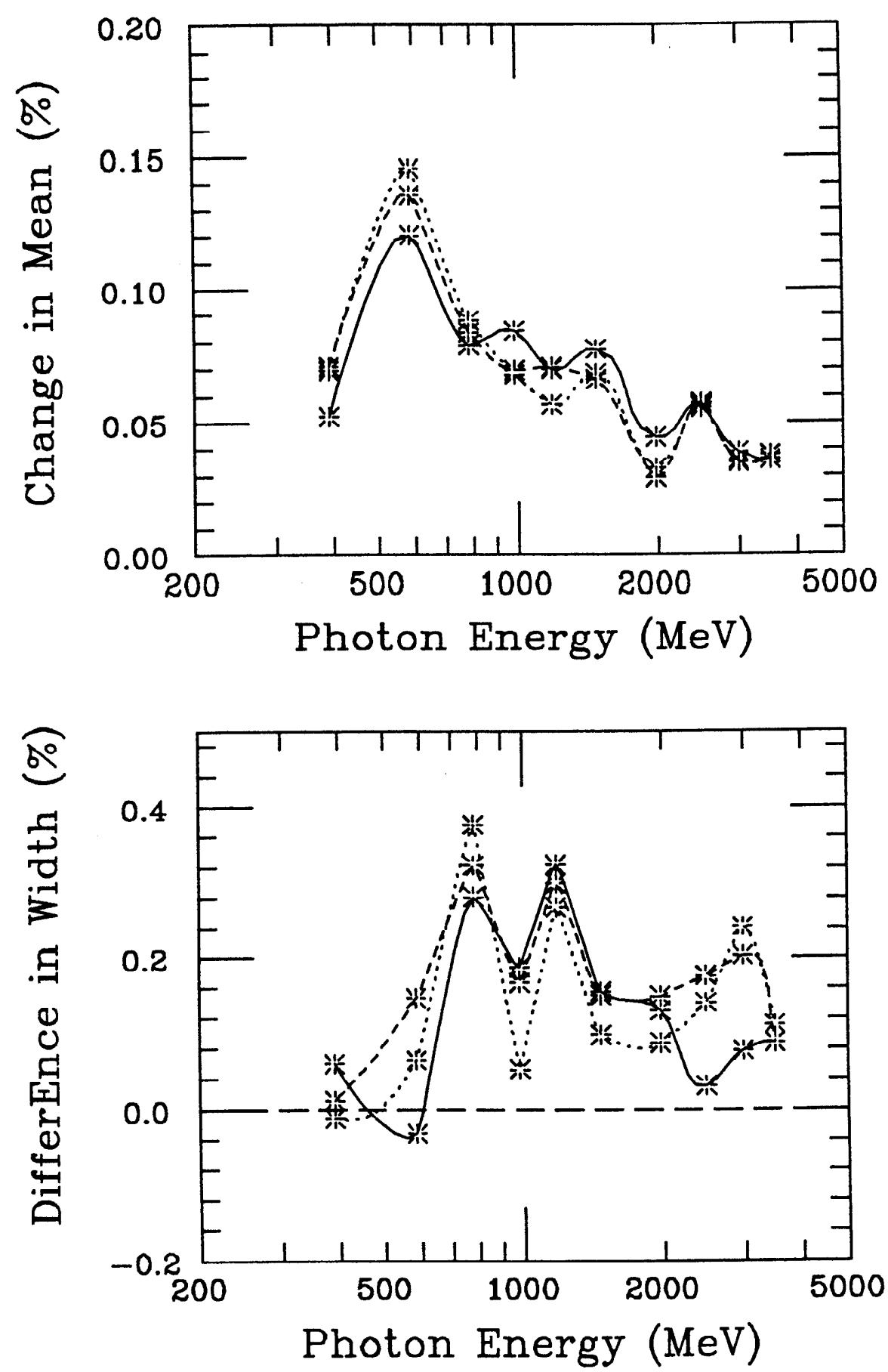

Fig. A.12 The change in the mean and sigma as modeled by CISSY. The solid curves correspond to the "soft" set of cuts, the dashed curves to the "medium" cuts and the dotted curves to the "hard" cuts. 


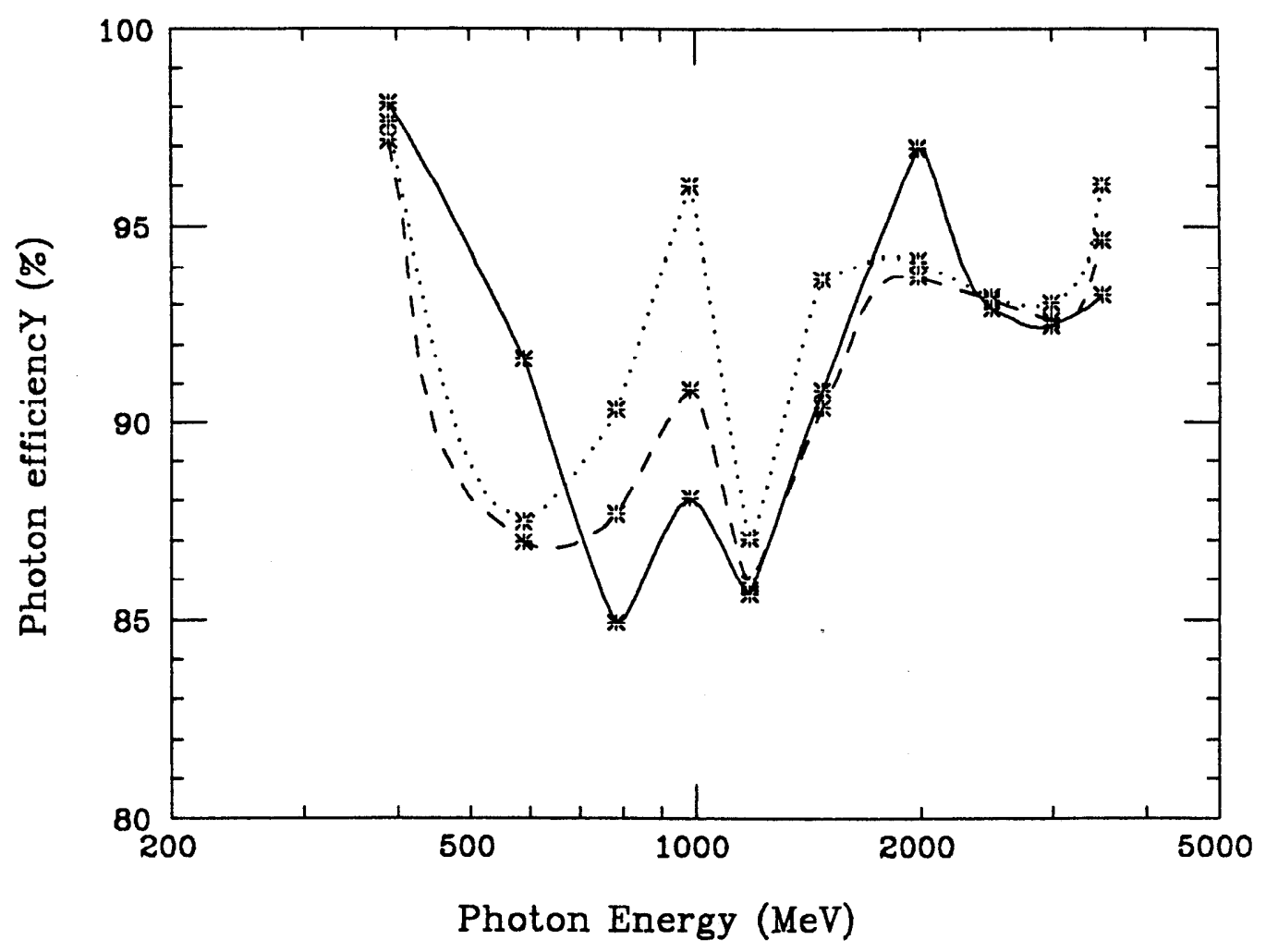

Fig. A.19 The photon efficiency as a function of energy for the 1984 data. The solid curve correspond to the "soft" set of cuts, the dashed curve to the "medium" cuts and the dotted curve to the "hard" cuts. A branching ratio of $1 \%$ is modeled with the appropriate background level for a sample of roughly $400,000 \Upsilon(1 S)$ events. Points with the same photon energy are highly correlated.

"hard" cuts which have a low photon efficiency but high background rejection. The cuts used are shown in Table A.2.

The photon spectrum, both before and after CISSY, for each of the three sets of cuts, was made and fitted in two passes. The first pass involved fitting the photon peak with a variable amplitude, mean and width. The top plot in Figure A.12 shows the percent change in the photon peak's mean caused by the electronics problem. For example, a $1 \mathrm{GeV}$ photon appears about $0.8 \mathrm{MeV}$ higher in energy than it should because of the malfunction. This shift is well below the Crystal Ball's absolute energy resolution and is therefore negligible. The bottom 
plot shows the difference in width before and after CISSY. The greatest difference is about $0.3 \%$ which implies the resolution is expected to rise from $2.7 \%$ to not more than about $3.0 \%$. This change would be important if any large signals were present in the real data set, but because no significant peaks are seen, the width is fixed at $2.7 \%$ for all upper limit calculations. Fixing the width to $2.7 \%$ has no measurable effect on the efficiency for small branching ratios as discussed below, but has the advantage that all $\Upsilon(1 S)$ data can be fit in the same manner.

In the second fitting pass, a statistical background similar to that found in the real data is added to a smaller sample of Monte Carlo events. The background level and peak size were selected to simulate a signal with about a $1 \%$ branching ratio in about $400,000 \Upsilon(1 \mathrm{~S})$ events. Care was taken to adjust the background level with the change in efficiency for the three sets of cuts. The mean was fixed to that found in the first pass with the full Monte Carlo data set and the width was fixed at $2.7 \%$. Figure A.13 shows the efficiency for the three sets of cuts. Because the same events are subjected to different cuts for each energy, the three points with the same energy are highly correlated. If the width is fixed to the value found in the first fitting pass, or if the fit after CISSY is fixed to the value found before CISSY, the results are almost identical; the points do not move by more than the size of their star pattern in Figure A.13. If backgrounds with the same shape but which are statistically different are used, Figure A.13 does not visibly change. The curve which corresponds to the "soft" cuts is applied to the efficiency calculation for the 1984 data set because the soft cuts most resemble those used in the analysis found in this thesis.

In summary, the electronics problem caused an inefficiency of about $15 \%$ for photons in the $1 \mathrm{GeV}$ energy range in the 1984 data set. The shift in the mean is negligible compared to the Crystal Ball absolute energy resolution. The increased width is too small to measurably affect the results presented here. The estimated inefficiency is used to correct the 1984 data in this thesis. 
Appendix B

\section{Information Useful for Combining Data Sets}

\section{B.1 Information Useful for Combining Data Sets}

Because of the great effort needed to experimentally reach the branching ratios predicted by the Wilczek calculation with $Q C D$ radiative corrections, the combining of data sets by more than one experiment will be the most direct route to a stronger result in the near future. If the results from this analysis and those from the Argus, CLEO and CUSB experiments discussed in Chapter 1 were combined, a Higgs particle in the $4-6 \mathrm{GeV} / \mathrm{c}^{2}$ mass range could probably be ruled out. Other experiments in the future could then improve on this result.

The difficulty in combining data sets is getting the information in usable form. Although the upper limit curves from other experiments are published, they cannot be combined to give a better upper limit; the fit results and detection efficiency as a function of photon energy are needed so the data can be combined correctly. To make the Crystal Ball data usable in such a program, Table B.1 lists the final photon efficiency, along with the amplitude and error from the fitting procedure, as a function of photon energy. The corresponding number of produced $\Upsilon(1 \mathrm{~S})$ events is $(4.36 \pm 0.28) \times 10^{5}$. Note that these efficiencies are model dependent; radiative decays to states which couple to fermions in proportion to the fermion mass are assumed here. Chapter 4 details how the upper limits were derived from these data. 


\begin{tabular}{|c|c|c|c|c|c|}
\hline $\log _{e}\left(E_{\gamma}\right)$ & $\begin{array}{c}\text { Photon } \\
\text { Efficiency (\%) }\end{array}$ & $\begin{array}{c}\text { Fit } \\
\text { Amplitude }\end{array}$ & $\log _{e}\left(E_{\gamma}\right)$ & $\begin{array}{c}\text { Photon } \\
\text { Efficiency (\%) }\end{array}$ & $\begin{array}{c}\text { Fit } \\
\text { Amplitude }\end{array}$ \\
\hline 6.10 & 15.8 & $30.6 \pm 94.3$ & 6.11 & 15.9 & $44.2 \pm 94.0$ \\
\hline 6.12 & 16.0 & $47.2 \pm 93.6$ & 6.13 & 16.1 & $39.5 \pm 93.3$ \\
\hline 6.14 & 16.2 & $24.3 \pm 93.0$ & 6.15 & 16.3 & $7.2 \pm 92.6$ \\
\hline 6.16 & 16.4 & $-6.3 \pm 92.3$ & 6.17 & 16.5 & $-12.1 \pm 91.8$ \\
\hline 6.18 & 16.6 & $-9.5 \pm 91.4$ & 6.19 & 16.7 & $0.0 \pm 90.9$ \\
\hline 6.20 & 16.8 & $12.2 \pm 90.4$ & 6.21 & 16.9 & $22.3 \pm 90.1$ \\
\hline 6.22 & 17.0 & $28.3 \pm 89.7$ & 6.23 & 17.1 & $30.8 \pm 89.3$ \\
\hline 6.24 & 17.2 & $31.0 \pm 89.0$ & 6.25 & 17.3 & $30.0 \pm 88.6$ \\
\hline 6.26 & 17.4 & $27.9 \pm 88.1$ & 6.27 & 17.5 & $23.8 \pm 87.6$ \\
\hline 6.28 & 17.6 & $16.2 \pm 86.9$ & 6.29 & 17.7 & $4.1 \pm 86.4$ \\
\hline 6.30 & 17.8 & $-12.4 \pm 86.0$ & 6.31 & 17.9 & $-31.5 \pm 85.5$ \\
\hline 6.32 & 18.0 & $-49.9 \pm 84.9$ & 6.33 & 18.1 & $-64.8 \pm 84.4$ \\
\hline 6.34 & 18.2 & $-75.2 \pm 83.8$ & 6.35 & 18.3 & $-81.2 \pm 83.3$ \\
\hline 6.36 & 18.4 & $-83.2 \pm 82.9$ & 6.37 & 18.5 & $-80.8 \pm 82.5$ \\
\hline 6.38 & 18.6 & $-71.9 \pm 82.2$ & 6.39 & 18.7 & $-54.8 \pm 81.8$ \\
\hline 6.40 & 18.8 & $-30.1 \pm 81.6$ & 6.41 & 19.0 & $-0.8 \pm 81.3$ \\
\hline 6.42 & 19.1 & $28.7 \pm 80.9$ & 6.43 & 19.2 & $54.0 \pm 80.7$ \\
\hline 6.44 & 19.4 & $71.9 \pm 80.4$ & 6.45 & 19.5 & $81.3 \pm 80.1$ \\
\hline 6.46 & 19.6 & $83.3 \pm 79.7$ & 6.47 & 19.8 & $80.2 \pm 79.3$ \\
\hline 6.48 & 19.9 & $74.1 \pm 78.8$ & 6.49 & 20.0 & $66.5 \pm 78.3$ \\
\hline 6.50 & 20.2 & $57.6 \pm 77.8$ & 6.51 & 20.3 & $46.7 \pm 77.2$ \\
\hline 6.52 & 20.4 & $33.1 \pm 76.7$ & 6.53 & 20.6 & $16.3 \pm 76.0$ \\
\hline 6.54 & 20.7 & $-3.6 \pm 75.4$ & 6.55 & 20.8 & $-24.5 \pm 74.9$ \\
\hline 6.56 & 21.0 & $-43.2 \pm 74.3$ & 6.57 & 21.1 & $-55.8 \pm 73.8$ \\
\hline
\end{tabular}




\begin{tabular}{|c|c|c|c|c|c|}
\hline 6.58 & 21.2 & $-58.8 \pm 73.4$ & 6.59 & 21.4 & $-51.6 \pm 73.0$ \\
\hline 6.60 & 21.5 & $-37.1 \pm 72.4$ & 6.61 & 21.6 & $-21.0 \pm 71.9$ \\
\hline 6.62 & 21.7 & $-9.0 \pm 71.4$ & 6.63 & 21.9 & $-5.4 \pm 71.0$ \\
\hline 6.64 & 22.0 & $-10.8 \pm 70.0$ & 6.65 & 22.1 & $-22.2 \pm 70.2$ \\
\hline 6.66 & 22.3 & $-33.3 \pm 69.7$ & 6.67 & 22.4 & $-38.0 \pm 69.2$ \\
\hline 6.68 & 22.6 & $-32.5 \pm 68.8$ & 6.69 & 22.7 & $-17.3 \pm 68.3$ \\
\hline 6.70 & 22.9 & $3.5 \pm 67.9$ & 6.71 & 23.1 & $23.7 \pm 67.4$ \\
\hline 6.72 & 23.2 & $38.2 \pm 67.0$ & 6.73 & 23.4 & $44.7 \pm 66.6$ \\
\hline 6.74 & 23.6 & $44.3 \pm 66.2$ & 6.75 & 23.7 & $39.1 \pm 65.7$ \\
\hline 6.76 & 23.9 & $30.7 \pm 65.1$ & 6.77 & 24.1 & $19.6 \pm 64.4$ \\
\hline 6.78 & 24.2 & $-10.4 \pm 63.6$ & 6.79 & 24.4 & $-26.8 \pm 63.1$ \\
\hline 6.80 & 24.6 & $-41.2 \pm 62.6$ & 6.81 & 24.7 & $-48.0 \pm 62.2$ \\
\hline 6.82 & 24.9 & $-42.8 \pm 61.7$ & 6.83 & 25.1 & $-26.9 \pm 61.1$ \\
\hline 6.84 & 25.2 & $-7.1 \pm 60.5$ & 6.85 & 25.4 & $8.3 \pm 60.1$ \\
\hline 6.86 & 25.6 & $12.7 \pm 59.5$ & 6.87 & 25.7 & $5.8 \pm \mathbf{5 9 . 0}$ \\
\hline 6.88 & 25.9 & $-6.9 \pm 58.6$ & 6.89 & 26.1 & $-17.9 \pm 58.1$ \\
\hline 6.90 & 26.2 & $-20.9 \pm 57.6$ & 6.91 & 26.2 & $-14.2 \pm 57.0$ \\
\hline 6.92 & 26.3 & $-1.2 \pm 56.4$ & 6.93 & 26.3 & $12.8 \pm 56.0$ \\
\hline 6.94 & 26.4 & $23.5 \pm 55.6$ & 6.95 & 26.4 & $29.4 \pm 55.2$ \\
\hline 6.96 & 26.5 & $31.1 \pm 54.7$ & 6.97 & 26.6 & $29.5 \pm 54.1$ \\
\hline 6.98 & 26.6 & $24.9 \pm 53.6$ & 6.99 & 26.7 & $16.3 \pm 52.9$ \\
\hline 7.00 & 26.7 & $2.9 \pm 52.2$ & 7.01 & 26.8 & $-13.1 \pm 51.6$ \\
\hline 7.02 & 26.8 & $-27.1 \pm 51.0$ & 7.03 & 26.9 & $-34.3 \pm 50.6$ \\
\hline 7.04 & 26.9 & $-31.7 \pm 50.2$ & 7.05 & 27.0 & $-19.8 \pm 49.7$ \\
\hline 7.06 & 27.0 & $-2.6 \pm 49.2$ & 7.07 & 27.1 & $14.0 \pm 48.9$ \\
\hline 7.08 & 27.2 & $25.1 \pm 48.5$ & 7.09 & 27.4 & $28.6 \pm 48.1$ \\
\hline
\end{tabular}




\begin{tabular}{|c|c|c|c|c|c|}
\hline 7.10 & 27.7 & $25.0 \pm 47.7$ & 7.11 & 27.9 & $16.6 \pm 47.1$ \\
\hline 7.12 & 28.1 & $6.4 \pm 46.6$ & 7.13 & 28.4 & $-3.3 \pm 46.1$ \\
\hline 7.14 & 28.6 & $-11.1 \pm 45.7$ & 7.15 & 28.9 & $-16.4 \pm 44.9$ \\
\hline 7.16 & 29.1 & $-19.1 \pm 44.6$ & 7.17 & 29.3 & $-19.0 \pm 44.2$ \\
\hline 7.18 & 29.6 & $-15.7 \pm 43.9$ & 7.19 & 29.8 & $-9.1 \pm 43.5$ \\
\hline 7.20 & 30.1 & $-0.5 \pm 43.2$ & .7 .21 & 30.3 & $6.7 \pm 42.7$ \\
\hline 7.22 & 30.5 & $9.0 \pm 42.6$ & 7.23 & 30.8 & $4.7 \pm 41.9$ \\
\hline 7.24 & 31.0 & $-4.4 \pm 41.5$ & 7.25 & 31.3 & $-14.4 \pm 41.1$ \\
\hline 7.26 & 31.5 & $-20.3 \pm 40.7$ & 7.27 & 31.7 & $-19.3 \pm 40.3$ \\
\hline 7.28 & 32.0 & $-11.3 \pm 40.0$ & 7.29 & 32.2 & $1.4 \pm 39.8$ \\
\hline 7.30 & 32.5 & $15.3 \pm 39.6$ & 7.31 & 32.6 & $28.4 \pm 39.5$ \\
\hline 7.32 & 32.8 & $37.0 \pm 39.1$ & 7.33 & 32.9 & $37.7 \pm 38.8$ \\
\hline 7.34 & 33.1 & $28.8 \pm 38.4$ & 7.35 & 33.3 & $11.3 \pm 37.9$ \\
\hline 7.36 & 33.4 & $-11.8 \pm 37.3$ & 7.37 & 33.6 & $-35.2 \pm 36.7$ \\
\hline 7.38 & 33.7 & $-53.9 \pm 36.2$ & 7.39 & 33.9 & $-63.5 \pm 35.8$ \\
\hline 7.40 & 34.0 & $-60.8 \pm 35.7$ & 7.41 & 34.2 & $-45.1 \pm 35.6$ \\
\hline 7.42 & 34.3 & $-20.9 \pm 35.5$ & 7.43 & 34.5 & $4.1 \pm 35.5$ \\
\hline 7.44 & 34.7 & $23.6 \pm 35.6$ & 7.45 & 34.8 & $34.3 \pm 35.6$ \\
\hline 7.46 & 35.0 & $36.9 \pm 35.5$ & 7.47 & 35.1 & $34.8 \pm 35.4$ \\
\hline 7.48 & 35.3 & $31.0 \pm 35.2$ & 7.49 & 35.5 & $26.4 \pm 34.9$ \\
\hline 7.50 & 35.6 & $20.5 \pm 34.4$ & 7.51 & 35.8 & $12.0 \pm 34.2$ \\
\hline 7.52 & 35.9 & $1.8 \pm 33.9$ & 7.53 & 36.1 & $-7.5 \pm 33.7$ \\
\hline 7.54 & 36.3 & $-13.2 \pm 33.3$ & 7.55 & 36.4 & $-13.7 \pm 33.1$ \\
\hline 7.56 & 36.6 & $-9.5 \pm 33.0$ & 7.57 & 36.7 & $-2.3 \pm 33.0$ \\
\hline 7.58 & 36.9 & $6.1 \pm 32.9$ & 7.59 & 37.1 & $13.3 \pm 32.9$ \\
\hline 7.60 & 37.2 & $16.3 \pm 32.8$ & 7.61 & 37.4 & $13.3 \pm 32.6$ \\
\hline
\end{tabular}




\begin{tabular}{|c|c|c|c|c|c|}
\hline 7.62 & 37.6 & $5.4 \pm 32.5$ & 7.63 & 37.7 & $-3.8 \pm 32.5$ \\
\hline 7.64 & 37.9 & $-9.8 \pm 32.3$ & 7.65 & 38.0 & $-10.6 \pm 32.1$ \\
\hline 7.66 & 38.2 & $-9.3 \pm 31.9$ & 7.67 & 38.4 & $-10.8 \pm 31.7$ \\
\hline 7.68 & 38.5 & $-17.3 \pm 31.6$ & 7.69 & 38.7 & $-26.5 \pm 31.6$ \\
\hline 7.70 & 38.9 & $-32.9 \pm 31.9$ & 7.71 & 39.0 & $-30.3 \pm 31.8$ \\
\hline 7.72 & 39.2 & $-17.7 \pm 31.9$ & 7.73 & 39.3 & $0.0 \pm 32.1$ \\
\hline 7.74 & 39.5 & $16.1 \pm 32.3$ & 7.75 & 39.7 & $25.5 \pm 32.6$ \\
\hline 7.76 & 39.8 & $26.6 \pm 32.7$ & 7.77 & 40.0 & $21.3 \pm 32.8$ \\
\hline 7.78 & 40.1 & $12.2 \pm 32.6$ & 7.79 & 40.3 & $-19.0 \pm 32.5$ \\
\hline 7.80 & 40.4 & $-25.4 \pm 32.5$ & 7.81 & 40.6 & $-28.0 \pm 32.4$ \\
\hline 7.82 & 40.8 & $-23.9 \pm 32.7$ & 7.83 & 41.1 & $-11.2 \pm 33.0$ \\
\hline 7.84 & 41.3 & $8.3 \pm 33.3$ & 7.85 & 41.6 & $28.4 \pm 33.5$ \\
\hline 7.86 & 41.9 & $41.8 \pm 33.6$ & 7.87 & 42.1 & $43.8 \pm 33.8$ \\
\hline 7.88 & 42.4 & $35.3 \pm 33.8$ & 7.89 & 42.7 & $22.0 \pm 33.9$ \\
\hline 7.90 & 43.0 & $11.6 \pm 33.9$ & 7.91 & 43.2 & $8.9 \pm 33.9$ \\
\hline 7.92 & 43.5 & $13.3 \pm 33.8$ & 7.93 & 43.8 & $20.3 \pm 33.9$ \\
\hline 7.94 & 44.1 & $26.4 \pm 34.2$ & 7.95 & 44.3 & $30.6 \pm 34.4$ \\
\hline 7.96 & 44.6 & $33.5 \pm 34.5$ & 7.97 & 44.9 & $36.4 \pm 34.8$ \\
\hline 7.98 & 45.1 & $38.9 \pm 34.9$ & 7.99 & 45.4 & $39.7 \pm 34.9$ \\
\hline 8.00 & 45.7 & $37.4 \pm 34.9$ & 8.01 & 45.9 & $30.5 \pm 34.8$ \\
\hline 8.02 & 46.2 & $17.9 \pm 34.8$ & 8.03 & 46.4 & $-0.5 \pm 34.5$ \\
\hline 8.04 & 46.6 & $-22.5 \pm 34.3$ & 8.05 & 46.8 & $-43.0 \pm 34.1$ \\
\hline 8.06 & 47.0 & $-55.2 \pm 34.1$ & 8.07 & 47.3 & $-55.2 \pm 34.1$ \\
\hline 8.08 & 47.5 & $-45.8 \pm 34.1$ & 8.09 & 47.7 & $-35.9 \pm 34.1$ \\
\hline 8.10 & 47.9 & $-31.3 \pm \mathbf{3 4 . 3}$ & 8.11 & 48.1 & $-31.6 \pm 34.5$ \\
\hline 8.12 & 48.4 & $-32.1 \pm 34.5$ & 8.13 & 48.6 & $-28.5 \pm 34.5$ \\
\hline
\end{tabular}




\begin{tabular}{|ccc|ccc|}
\hline 8.14 & 48.8 & $-20.8 \pm 34.6$ & 8.15 & 49.0 & $-11.3 \pm 34.8$ \\
\hline 8.16 & 49.1 & $-3.7 \pm 34.9$ & 8.17 & 49.0 & $-1.9 \pm 34.5$ \\
\hline 8.18 & 48.9 & $-7.3 \pm 35.0$ & 8.19 & 48.8 & $-17.1 \pm 34.1$ \\
\hline 8.20 & 48.6 & $-25.6 \pm 33.8$ & 8.21 & 48.5 & $-27.8 \pm 33.5$ \\
\hline 8.22 & 48.4 & $-22.6 \pm 33.4$ & 8.23 & 47.9 & $-12.4 \pm 33.2$ \\
\hline 8.24 & 46.9 & $-1.5 \pm 32.8$ & 8.25 & 46.0 & $5.2 \pm 32.5$ \\
\hline 8.26 & 39.1 & $5.5 \pm 32.2$ & 8.27 & 28.2 & $1.9 \pm 31.6$ \\
\hline 8.28 & 17.4 & $0.9 \pm 31.3$ & 8.29 & 6.4 & $8.1 \pm 30.9$ \\
\hline 8.30 & 8.9 & $21.8 \pm 30.3$ & 8.31 & 30.3 & $35.1 \pm 29.7$ \\
\hline 8.32 & 28.3 & $43.1 \pm 29.1$ & 8.33 & 26.2 & $45.6 \pm 28.2$ \\
\hline 8.34 & 24.2 & $45.3 \pm 27.7$ & 8.35 & 22.2 & $42.8 \pm 26.5$ \\
\hline 8.36 & 20.1 & $35.3 \pm 25.4$ & 8.37 & 18.1 & $22.8 \pm 24.1$ \\
\hline 8.38 & 16.1 & $8.8 \pm 22.8$ & 8.39 & 14.2 & $-1.8 \pm 21.2$ \\
\hline 8.40 & 12.7 & $-8.0 \pm 19.4$ & 8.41 & 11.1 & $-13.3 \pm 17.8$ \\
\hline 8.42 & 9.6 & $-18.8 \pm 16.3$ & 8.43 & 8.1 & $-20.4 \pm 14.7$ \\
\hline 8.44 & 6.6 & $-15.7 \pm 12.9$ & 8.45 & 0.6 & $-8.6 \pm 11.4$ \\
\hline 8.46 & 0.0 & $-3.0 \pm 9.8$ & & & \\
\hline
\end{tabular}

Table B.1 The numerical quantities used in the upper limit calculation as a function of photon energy. 